Foundations and Trends ${ }^{\circledR}$ in

Signal Processing

Vol. 2, No. 1 (2008) 1-94

(C) 2008 J. Kovačević and A. Chebira

DOI: $10.1561 / 2000000006$

the essence of knowledge

\title{
An Introduction to Frames
}

\section{Jelena Kovačević ${ }^{1}$ and Amina Chebira ${ }^{2}$}

1 Center for Bioimage Informatics, Carnegie Mellon University, 5000 Forbes Avenue, PA 15213,USA, j.kovacevic@ieee.org

2 Center for Bioimage Informatics, Carnegie Mellon University, 5000

Forbes Avenue, PA 15213, USA, achebira@ieee.org

\begin{abstract}
This survey gives an introduction to redundant signal representations called frames. These representations have recently emerged as yet another powerful tool in the signal processing toolbox and have become popular through use in numerous applications. Our aim is to familiarize a general audience with the area, while at the same time giving a snapshot of the current state-of-the-art.
\end{abstract}

\footnotetext{
* Based on "Life Beyond Bases: The Advent of Frames (Parts I and II)," by Jelena Kovačević and Amina Chebira, (C) IEEE Signal Processing Magazine, 2007.
} 


\section{Introduction}

Redundancy is a common tool in our daily lives. We double- and triplecheck that we turned off gas and lights, took our keys, money, etc. (at least those worrywarts among us do). When an important date is coming up, we drive our loved ones crazy by confirming "just once more" they are on top of it.

The same idea of removing doubt is present in signal representations. Given a signal, we represent it in another system, typically a basis, where its characteristics are more readily apparent in the transform coefficients. However, these representations are typically nonredundant, and thus corruption or loss of transform coefficients can be serious. In comes redundancy; we build a safety net into our representation so that we can avoid those disasters. The redundant counterpart of a basis is called a frame. ${ }^{1}$

It is generally acknowledged ${ }^{2}$ that frames were born in 1952 in the work of Duffin and Schaeffer [78]. Despite being over half a century old, frames gained popularity only in the last decade, due mostly to the work of the three wavelet pioneers - Daubechies et al. [67].

${ }^{1}$ No one seems to know why they are called frames, perhaps because of the bounds in (3.8).

${ }^{2}$ At least in the signal processing and harmonic analysis communities. 
Frame-like ideas, that is, building redundancy into a signal expansion, can be found in pyramid coding [33], resilience to noise [18, 19, 60, 64, 65, 93, 98, 133], denoising [53, 77, 88, 110, 177], robust transmission $[20,21,22,25,41,92,105,139,157,165]$, CDMA systems [131, 161, 168, 169], multiantenna code design [100, 104], segmentation [69, 124, 162], classification [48, 124, 162], prediction of epileptic seizures [16, 17], restoration and enhancement [113], motion estimation [128], signal reconstruction [6], coding theory [101, 143], operator theory [2], quantum theory and computing [80, 151, 153], and many others.

While frames are often associated with wavelet frames, frames are more general than that. Wavelet frames possess structure; frames are redundant representations that only need to represent signals in a given space with a certain amount of redundancy. The simplest frame, appropriately named Mercedes-Benz, is introduced in Figure 3.2; just have a peek now, we will go into more details later.

Why and where would one use frames? The answer is simple: anywhere where redundancy is a must. The host of the applications mentioned above and discussed later in the survey illustrate that richly.

Now a word about what you are reading: why an introductory survey? The sources on frames are the beautiful book by Daubechies [64], a recent book by Christensen [51] as well as a number of classic papers [39, 63, 99, 103], among others. Although excellent material, none of the above sources offer an introduction to frames geared primarily to engineers and those who just want an introduction into the area. Thus our emphasis; this is a survey, rather than a comprehensive survey of the state of the field. Although we will touch upon a number of applications and theoretical results, we will do so only for the sake of teaching. We will go slowly, whenever possible using the simplest examples. Generalizations will follow naturally. We will be selective and will necessarily give our personal view of frames. We will be rigorous when necessary; however, we will not insist upon it at all times. As often as possible, we will be living in the finite-dimensional world; it is rich enough to give a flavor of the basic concepts. When we do venture into the infinite-dimensional one, we will do so only using 


\section{Introduction}

filter banks — structured expansions used in many signal processing applications.

This treatment is largely reproduced from two tutorials published in the IEEE Signal Processing Magazine [117, 118]. The aim here is to present the material in one piece, with more detail and ease of referencing. 


\section{Review of Bases}

The reason we try to represent our signals in a different domain is, typically, because certain signal characteristics become obvious in that other domain, facilitating various signal processing tasks. For example, we perform Fourier analysis to uncover the harmonic composition of a signal. If our signal happens to be a sum of a finite number of tones, the Fourier-domain representation will be nonzero at exactly those tones and will be zero at all other frequencies. However, if our signal is a sum of, say a pure frequency and a pulse of very short duration, the Fourier transform will be an inefficient representation ${ }^{1}$; the signal energy will be, more or less, spread evenly across all frequencies. Thus, the right representation is absolutely critical if we are to perform our signal processing task effectively and efficiently.

To understand frames, it helps to go back to what we already know: bases. In this section, we review essential concepts on bases (we assume basic notions on vector spaces, inner products, norms). If you are familiar with those, you may skip this section and go directly to the frame section which comes next. We stress that often, we will forgo formal

\footnotetext{
${ }^{1}$ We assume here that the quality of the representation is measured by its sparsity.
} 
language in favor of making the material as accessible as possible. An introductory treatment is also given in [167].

When modeling a problem, one needs to identify a space of objects on which certain operations will be performed. For example, in image compression, our objects are images, while in some other tasks, our objects can be audio signals, movies, and many others. Initially, we will assume that these objects are vectors in a vector space. In this survey, we consider almost exclusively finite-dimensional vector spaces $\mathbb{R}^{n}$ and $\mathbb{C}^{n}$, as well as the infinite-dimensional vector space $\ell^{2}(\mathbb{Z})$ (commonly used in discrete-time signal processing). By itself, a vector space will not afford much, except for the ability to add two vectors to form a new vector in the same vector space and to multiply a vector by a scalar. To do anything meaningful, we must equip such a space with an inner product and a norm, which will allow us to "measure" things. These functions turn the vector space into an inner product space. By introducing the distance between two vectors, as the norm of the difference between those two vectors, we get a precise measurement tool and turn our inner product space into a metric space. Finally, by considering the question of completeness, that is, whether a representative set of vectors can describe every other vector from our space, we reach the Hilbert space stage, which we denote by $\mathbb{H}$. This progression allows us to do things such as measure similarity between two images by finding the distance between them, a step present in compression algorithms, systems for retrieval and matching, and many others.

We need even more: tools which will allow us to look at all the vectors in a common representation system. These tools already exist as bases in a Hilbert space. Bases are sets of vectors used to uniquely represent any vector in a given Hilbert space in terms of the basis vectors. An orthonormal basis, in particular, will allow us not only to represent vectors but to approximate them as well. ${ }^{2}$ This is useful when resources do not allow us to deal with the object directly, but rather with its approximation only. For example, an "instant" approximation of a natural image is just its lowpassed version — we get a blurry image.

\footnotetext{
${ }^{2}$ Note that approximations are possible in all types of bases, not just orthogonal ones.
} 
A subset $\Phi=\left\{\varphi_{i}\right\}_{i \in I}$ of a finite-dimensional vector space $\mathbb{V}$ (where $I$ is some index set) is called a basis for $\mathbb{V}$, if $\mathbb{V}=\operatorname{span}(\Phi)^{3}$ and the vectors in $\Phi$ are linearly independent. If $I=\{1, \ldots, n\}$, we say that $\mathbb{V}$ has dimension $n$.

A vector space $\mathbb{V}$ is infinite dimensional if it contains an infinite linearly independent set of vectors. ${ }^{4}$ If $\mathbb{V}$ is equipped with a norm, then a subset $\Phi=\left\{\varphi_{i}\right\}_{i \in I}$ of $\mathbb{V}$ is called a basis ${ }^{5}$ if for every $x$ in $\mathbb{V}$, there exist unique scalars $X_{i}$ such that $x=\sum_{i \in I} X_{i} \varphi_{i}$.

As we already mentioned, in this survey, we consider exclusively the finite-dimensional Hilbert spaces $\mathbb{H}=\mathbb{R}^{n}, \mathbb{C}^{n}$ with $I=\{1, \ldots, n\}$, as well as the infinite-dimensional space of square-summable sequences $\mathbb{H}=\ell^{2}(\mathbb{Z})$ with $I=\mathbb{Z}$.

$\mathbb{R}^{n}$ and $\mathbb{C}^{n}$ are the most intuitive Hilbert spaces which we deal with on a daily basis. Their dimension is $n$. For example, the complex space $\mathbb{C}^{n}$ is the set of all $n$-tuples $x=\left(x_{1}, \ldots, x_{n}\right)^{T}$, with $x_{i}$ in $\mathbb{C}$ (similarly for $\mathbb{R}^{n}$ ) and where $T$ denotes the transpose.

In discrete-time signal processing we deal almost exclusively with sequences $x$ having finite square sum or finite energy, where $x=$ $\left(\ldots, x_{-1}, x_{0}, x_{1}, \ldots\right)$ is, in general, complex-valued. Such a sequence $x$ is a vector in the Hilbert space $\ell^{2}(\mathbb{Z})$.

For the above spaces, the inner product between two vectors $x$ and $y$ is defined as ${ }^{6}$ :

$$
\langle x, y\rangle=\sum_{i \in I} x_{i} y_{i}^{*}
$$

\footnotetext{
${ }^{3}$ Given $S \subset \mathbb{V}$, the span of $S$ is the subspace of $\mathbb{V}$ consisting of all finite linear combinations of vectors in $S$.

${ }^{4}$ Many subtleties arise in infinite dimensions that are not present in finite dimensions, such as the proper use of "span" and "independence" as both of these words imply finite linear combinations. We do not address these issues here; instead, we refer the reader to [102].

${ }^{5}$ Also called a Schauder basis. Note that here we need a normed vector space because the definition implicitly uses the notion of convergence: the series converges to the vector $x$ in the norm of $\mathbb{V}$. Again, in the definition of a basis, we have to pay attention to the use of terms "span" and "independence" when we deal with infinite-dimensional spaces. For instance, the infinite set $\left\{\delta_{i-k}\right\}_{k \in \mathbb{Z}}$ is a Schauder basis for $\ell^{2}(\mathbb{Z})$ but does not $\operatorname{span} \ell^{2}(\mathbb{Z})$ because we cannot write every square-summable sequence as a finite linear combination of $\delta_{i}$ 's. For more details, we refer the reader to [102].

${ }^{6}$ We have used the convention that is dominant in mathematics, where the inner product is linear in the first argument and conjugate-linear in the second argument.
} 
where $*$ denotes Hermitian transposition, while the norm is

$$
\|x\|=\sqrt{\langle x, x\rangle}=\sqrt{\sum_{i \in I}\left|x_{i}\right|^{2}} .
$$

\subsection{Orthonormal Bases}

A basis $\Phi=\left\{\varphi_{i}\right\}_{i \in I}$, where the vectors are orthonormal:

$$
\left\langle\varphi_{i}, \varphi_{j}\right\rangle=\delta_{i-j}
$$

is called an orthonormal basis. Here $\delta_{i}$ denotes the Kronecker delta, where $\delta_{i}=1$ for $i=0$, and is 0 otherwise. In other words, an orthonormal system is called an orthonormal basis for $\mathbb{H}$, if for every $x$ in $\mathbb{H}$,

$$
x=\sum_{i \in I} X_{i} \varphi_{i}
$$

for some scalars $X_{i}$. These scalars are called the transform or expansion coefficients of $x$ with respect to $\Phi$, and it follows from orthonormality that they are given by

$$
X_{i}=\left\langle x, \varphi_{i}\right\rangle,
$$

for all $i \in I$. In fact, (2.2) can be derived from (2.1) by taking the inner products on both sides of $(2.1)$ with respect to $\varphi_{j}$.

We now discuss a few properties of orthonormal bases.

\subsubsection{Projections}

A characteristic of orthonormal bases allowing us to approximate signals is that an orthogonal projection onto a subspace spanned by a subset of basis vectors, $\left\{\varphi_{i}\right\}_{i \in J}$ ( $J$ is the index set of that subset), is

$$
P x=\sum_{i \in J}\left\langle x, \varphi_{i}\right\rangle \varphi_{i},
$$

that is, it is a sum of projections onto individual one-dimensional subspaces spanned by each $\varphi_{i}$. Beware that this is not true when $\left\{\varphi_{i}\right\}_{i \in J}$ do not form an orthonormal system. It is easy to show that (2.3) is indeed a projection by demonstrating that $P(P x)=P x$ and $P^{*} x=P x$; we 
leave it as an exercise. The above is a direct consequence of $\left\{\varphi_{i}\right\}_{i \in J}$ forming an orthonormal system. For example, projecting $x$ onto the subspace spanned by $\varphi_{1}$ and $\varphi_{2}$ would be

$$
\begin{aligned}
P x & =\left\langle x, \alpha \varphi_{1}+\beta \varphi_{2}\right\rangle\left(\alpha \varphi_{1}+\beta \varphi_{2}\right) \\
& =\left\langle x, \alpha \varphi_{1}+\beta \varphi_{2}\right\rangle \alpha \varphi_{1}+\left\langle x, \alpha \varphi_{1}+\beta \varphi_{2}\right\rangle \beta \varphi_{2} \\
& =\alpha^{2}\left\langle x, \varphi_{1}\right\rangle \varphi_{1}+\beta^{2}\left\langle x, \varphi_{2}\right\rangle \varphi_{2} .
\end{aligned}
$$

\subsubsection{Bessel's Inequality and Parseval's Equality}

Given an orthonormal system of vectors $\left\{\varphi_{i}\right\}_{i \in J}$ in $\mathbb{H}$, then, for every $x$ in $\mathbb{H}$, the following inequality, known as Bessel's inequality, holds:

$$
\sum_{i \in J}\left|\left\langle x, \varphi_{i}\right\rangle\right|^{2} \leq\|x\|^{2}
$$

This inequality follows from (2.3) since its left-hand side is $|\langle x, P x\rangle|=$ $\|P x\|^{2}$.

Given an orthonormal system that is complete in $\mathbb{H}$, then we have an orthonormal basis for $\mathbb{H}$, and Bessel's relation becomes an equality, often called Parseval's equality (or Plancherel's). This is simply the norm-preserving property of orthonormal bases. In other words

$$
\|x\|^{2}=\sum_{i \in I}\left|\left\langle x, \varphi_{i}\right\rangle\right|^{2} .
$$

As an example, in the case of the Fourier series, (2.4) becomes

$$
\|x\|^{2}=\sum_{k \in \mathbb{Z}}\left|X_{k}\right|^{2}
$$

where $X_{k}$ are Fourier coefficients.

\subsubsection{Least-Squares Approximation}

Suppose that we want to approximate a vector from a Hilbert space $\mathbb{H}$ by a vector $\hat{x}$ lying in the (closed) subspace $S=\operatorname{span}\left\{\varphi_{i}\right\}_{i \in J}$. The orthogonal projection of $x \in \mathbb{H}$ onto $S$ is given by (2.3). The difference vector $d=x-\hat{x}$ satisfies $d \perp S .^{7}$ This approximation is best in the

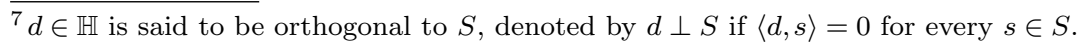


least-squares sense, that is, $\min \|x-y\|$ for $y$ in $S$ is attained for $y=\sum_{i} X_{i} \varphi_{i}$ with $X_{i}=\left\langle x, \varphi_{i}\right\rangle$ being the expansion coefficients. In other words, the best approximation is our $\hat{x}=P x$ previously defined in (2.3). An immediate consequence of this result is the successive approximation property of orthogonal expansions. Call $\hat{x}^{(k)}$ the best approximation of $x$ on the subspace spanned by $\left\{\varphi_{1}, \varphi_{2}, \ldots, \varphi_{k}\right\}$. Then the approximation $\hat{x}^{(k+1)}$ is given by

$$
\hat{x}^{(k+1)}=\hat{x}^{(k)}+\left\langle x, \varphi_{k+1}\right\rangle \varphi_{k+1},
$$

that is, the previous approximation plus the projection along the added vector $\varphi_{k+1}$.

The successive approximation property does not hold for nonorthogonal bases; When calculating the approximation $\hat{x}^{(k+1)}$, one cannot simply add one term to the previous approximation, but has to recalculate the whole approximation.

\subsubsection{Matrix View}

While we are great fans of equations, we like matrices even better, as equations can be hard to parse. Moreover, visualizing representations is more intuitive and helps us understand the concepts better. Thus, we rephrase our basis notions in matrix notation.

Example 2.1. Suppose we are given an orthonormal basis $\Phi=$ $\left\{(1,-1)^{T} / \sqrt{2},(1,1)^{T} / \sqrt{2}\right\}$. Given this basis and an arbitrary vector $x$ in the plane, what is this point in this new basis (new coordinate system)? We answer this question by projecting $x$ onto the new basis. Suppose that $x=(1,0)^{T}$. Then, $x_{\Phi_{1}}=\left\langle x, \varphi_{1}\right\rangle=1 / \sqrt{2}$ and $x_{\Phi_{2}}=\left\langle x, \varphi_{2}\right\rangle=1 / \sqrt{2}$. Thus, in this new coordinate system, our point $(1,0)^{T}$ becomes $x_{\Phi}=\left(x_{\Phi_{1}}, x_{\Phi_{2}}\right)^{T}=(1,1)^{T} / \sqrt{2}$. It is still the same point in the plane, we only read its coordinates depending on which basis we are considering. We can express the above process of figuring out the coordinates in the new coordinate system a bit more elegantly:

$$
\begin{aligned}
X & =x_{\Phi}=\left(\begin{array}{l}
x_{\Phi_{1}} \\
x_{\Phi_{2}}
\end{array}\right)=\left(\begin{array}{l}
\left\langle x, \varphi_{1}\right\rangle \\
\left\langle x, \varphi_{2}\right\rangle
\end{array}\right)=\left(\begin{array}{c}
\varphi_{11} x_{1}+\varphi_{12} x_{2} \\
\varphi_{21} x_{1}+\varphi_{22} x_{2}
\end{array}\right) \\
& =\left(\begin{array}{ll}
\varphi_{11} & \varphi_{12} \\
\varphi_{21} & \varphi_{22}
\end{array}\right)\left(\begin{array}{l}
x_{1} \\
x_{2}
\end{array}\right)=\frac{1}{\sqrt{2}}\left(\begin{array}{cc}
1 & -1 \\
1 & 1
\end{array}\right)\left(\begin{array}{l}
x_{1} \\
x_{2}
\end{array}\right)=\Phi^{*} x
\end{aligned}
$$


Observe that the matrix $\Phi$ describes an orthonormal basis in the real plane. ${ }^{8}$ The columns of the matrix are the basis vectors (the rows are as well), that is, the process of finding coordinates of a vector in a different coordinate system can be conveniently represented using a matrix $\Phi$ whose columns are the new basis vectors, $x_{\Phi}=\Phi^{*} x$.

We now summarize what we learned in this example in a more general case: Any Hilbert space basis (orthonormal or biorthogonal) can be represented as a matrix having basis vectors as its columns. If the matrix is singular, it does not represent a basis.

Given that we have $X=\Phi^{*} x$, we can go back to $x$ by inverting $\Phi^{*}$ (this is why we require $\Phi$ to be nonsingular), $x=\left(\Phi^{*}\right)^{-1} X$. If the original basis is orthonormal, then $\Phi$ is unitary and $\Phi^{-1}=\Phi^{*}$. The representation formula can then be written as

$$
x=\sum_{i \in I}\left\langle x, \varphi_{i}\right\rangle \varphi_{i}=\Phi \Phi^{*} x=\Phi^{*} \Phi x .
$$

Example 2.2 (DFT as an Orthonormal Basis Expansion). The discrete Fourier transform (DFT) is ubiquitous; however, it is not traditionally looked upon as a signal expansion or written in matrix form. The easiest way to do that is to look at how the reconstruction is obtained:

$$
x_{k}=\frac{1}{n} \sum_{i=0}^{n-1} X_{i} W_{n}^{i k}, \quad k=0, \ldots, n-1,
$$

where $W_{n}=e^{j 2 \pi / n}$ is an $n$th root of unity. In matrix notation we could write it as ${ }^{9}$

$$
x=\frac{1}{n} \underbrace{\left(\begin{array}{cccc}
1 & 1 & \cdots & 1 \\
1 & W_{n} & \cdots & W_{n}^{n-1} \\
\vdots & \vdots & \vdots & \vdots \\
1 & W_{n}^{n-1} & \cdots & W_{n}
\end{array}\right)}_{\Phi=\text { DFT T }_{n}^{*}} \underbrace{\left(\begin{array}{c}
X_{0} \\
X_{1} \\
\vdots \\
X_{n-1}
\end{array}\right)}_{X} .
$$

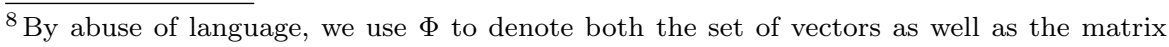
representing those vectors.

${ }^{9}$ Following our convention, $\Phi$ should be called DFT; however, as the standard in signal processing is the reverse, we use that notation.
} 
Note that the DFT matrix defined as above is not normalized, that is $(1 / n)\left(\mathrm{DFT}_{n}\right)^{*}\left(\mathrm{DFT}_{n}\right)=I$. If we normalize it by $1 / \sqrt{n}$, the DFT would exactly implement an orthonormal basis.

The decomposition formula is usually given as

$$
X_{i}=\sum_{k=0}^{n-1} x_{k} W_{n}^{-i k}, \quad i=0, \ldots, n-1
$$

and, in matrix notation:

$$
X=\mathrm{DFT}_{n} x .
$$

Consider now the normalized version. In basis parlance, our basis would be $\Phi=\left\{\varphi_{i}\right\}_{i=0}^{n-1}$, where the basis vectors are:

$$
\varphi_{i}=\frac{1}{\sqrt{n}}\left(W_{n}^{0}, W_{n}^{i}, \ldots, W_{n}^{i(n-1)}\right)^{T}, \quad i=0, \ldots, n-1 .
$$

Then, the expansion formula (2.8) can be seen as

$$
X_{i}=\left\langle x, \varphi_{i}\right\rangle, \quad i=0, \ldots, n-1,
$$

and the reconstruction formula $(2.7)$ for $x=\left(x_{0}, \ldots, x_{n-1}\right)^{T}$ :

$$
x=\sum_{i=0}^{n-1} X_{i} \varphi_{i}=\sum_{i=0}^{n-1}\left\langle x, \varphi_{i}\right\rangle \varphi_{i}=\underbrace{\frac{1}{\sqrt{n}} \operatorname{DFT}_{n}^{*}}_{\Phi} \underbrace{\frac{1}{\sqrt{n}} \operatorname{DFT}_{n} x}_{\Phi^{*}} .
$$

\subsection{General Bases}

We are now going to relax the constraint of orthogonality and see what happens. The reasons for doing that are numerous, the most obvious one being that we have more freedom in choosing our basis vectors. For example, in $\mathbb{R}^{2}$, once a vector is chosen, to get an orthonormal basis, we basically have only one choice (within a sign); on the other hand, for a general basis, it is enough to choose the second vector not collinear to the first. 
Example 2.3. As a simple example, consider the following set in $\mathbb{R}^{2}: \Phi=\left\{\varphi_{1}, \varphi_{2}\right\}=\left\{(1,0)^{T},(\sqrt{2} / 2, \sqrt{2} / 2)^{T}\right\}$. We have seen how orthonormal bases expand vectors. This is not an orthonormal basis but can we still use these two vectors to represent any real vector $x$ ? The answer is yes:

$$
x=\left\langle x, \tilde{\varphi}_{1}\right\rangle \varphi_{1}+\left\langle x, \tilde{\varphi}_{2}\right\rangle \varphi_{2},
$$

with $\tilde{\varphi}_{1}=(1,-1)$ and $\tilde{\varphi}_{2}=(0, \sqrt{2})$. Thus, we can represent any real vector with our initial pair of vectors $\Phi=\left\{\varphi_{1}, \varphi_{2}\right\}$; however, they need helpers, an extra pair of vectors $\tilde{\Phi}=\left\{\tilde{\varphi}_{1}, \tilde{\varphi}_{2}\right\}$.

So what can we say about these two couples? It is obvious that they work in concert to represent $x$. Further, while not orthogonal within the couple, they are orthogonal across couples; $\varphi_{1}$ is orthogonal to $\tilde{\varphi}_{2}$, while $\varphi_{2}$ is orthogonal to $\tilde{\varphi}_{1}$. Finally, the inner products between corresponding vectors in a couple are $\left\langle\varphi_{i}, \tilde{\varphi}_{i}\right\rangle=1$ for $i=1,2$.

In general, these biorthogonality relations can be compactly represented as

$$
\left\langle\varphi_{i}, \tilde{\varphi}_{j}\right\rangle=\delta_{i-j}
$$

The representation expression can then be written as

$$
x=\sum_{i \in I}\left\langle x, \tilde{\varphi}_{i}\right\rangle \varphi_{i}=\sum_{i \in I}\left\langle x, \varphi_{i}\right\rangle \tilde{\varphi}_{i},
$$

that is, the roles of $\varphi_{i}$ and $\tilde{\varphi}_{i}$ are interchangeable. These two sets of vectors, $\Phi$ and $\tilde{\Phi}$, are called biorthogonal bases and are said to be dual to each other. If the dual basis $\tilde{\Phi}$ is the same as $\Phi$, we get an orthonormal basis. Thus, orthonormal bases are self dual.

While orthonormal bases are norm preserving, that is, they satisfy Parseval's equality, this is not true in the biorthogonal case. This is one of the reasons successive approximation does not work here. In the orthonormal case, the norm of the original vector is sliced up into pieces, each of which is the norm of the corresponding expansion coefficient (and equal to the length of the appropriate projection). Here, we know that does not work. 


\section{Review of Bases}

From the above discussion, we see that biorthogonal bases offer a larger choice, since they are less constrained than the orthonormal ones. However, this comes at the price of losing the norm-preserving property as well as the successive approximation property. This trade-off is often tackled in practice and depending on the problem at hand, you might decide to use either orthonormal or biorthogonal basis.

\subsubsection{Matrix View}

When the original basis is biorthogonal, there is not much more we can

say about $\Phi$. The representation formula is (the two bases $\Phi$ and $\tilde{\Phi}$ are interchangeable):

$$
x=\sum_{i \in I}\left\langle x, \tilde{\varphi}_{i}\right\rangle \varphi_{i}=\Phi \tilde{\Phi}^{*} x=\tilde{\Phi} \Phi^{*} x=\sum_{i \in I}\left\langle x, \varphi_{i}\right\rangle \tilde{\varphi}_{i} .
$$




\section{Frame Definitions and Properties}

The notion of bases in finite-dimensional spaces implies that the number of representative vectors is the same as the dimension of the space. When this number is larger, we can still have a representative set of vectors, except that the vectors are no longer linearly independent and the resulting set is then called a frame. Frames are signal representation tools which are redundant, and since they are less constrained than bases, they are used when more flexibility in choosing a representation is needed.

In this section, we introduce frames through simple examples and consider $\mathbb{H}=\mathbb{R}^{n}, \mathbb{C}^{n}$ only. We then define frames more formally and discuss a number of their properties. In Section 4, we examine finitedimensional frames in some detail. Then, in Section 5, we look at the only instance of infinite-dimensional frames we discuss in this survey, those in $\mathbb{H}=\ell^{2}(\mathbb{Z})$ implemented using filter banks.

Example 3.1. Let us take an orthonormal basis, add a vector to it and see what happens. Suppose our system is as given in Figure 3.1(a), with $\Phi=\left\{\varphi_{1}, \varphi_{2}, \varphi_{3}\right\}=\left\{(1,0)^{T},(0,1)^{T},(1,-1)^{T}\right\}$. The first two vectors $\varphi_{1}, \varphi_{2}$ are the ones forming the orthonormal basis and the third one, 


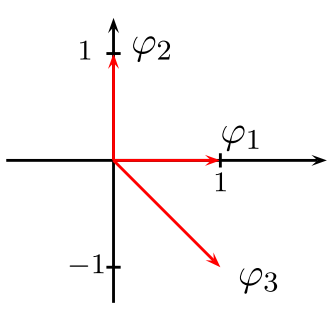

(a)

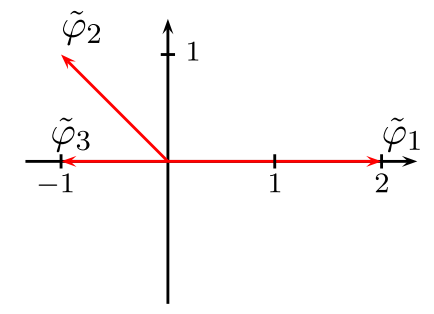

(b)

Fig. 3.1 A pair of general frames. (a) Frame $\Phi=\left\{\varphi_{1}, \varphi_{2}, \varphi_{3}\right\}$. (b) Dual frame $\tilde{\Phi}=$ $\left\{\tilde{\varphi}_{1}, \tilde{\varphi}_{2}, \tilde{\varphi}_{3}\right\}$.

$\varphi_{3}$, was added to the orthonormal basis. What can we say about such a system?

First, it is clear that by having three vectors in $\mathbb{R}^{2}$, those vectors must necessarily be linearly dependent; indeed, $\varphi_{3}=\varphi_{1}-\varphi_{2}$. It is also clear that these three vectors must be able to represent every vector in $\mathbb{R}^{2}$ since their subset is able to do so (which also means that we could have added any other vector $\varphi_{3}$ to our orthonormal basis with the same result.) In other words, since we know that the following is true,

$$
x=\left\langle x, \varphi_{1}\right\rangle \varphi_{1}+\left\langle x, \varphi_{2}\right\rangle \varphi_{2},
$$

we can add a zero to the above expression,

$$
x=\left\langle x, \varphi_{1}\right\rangle \varphi_{1}+\left\langle x, \varphi_{2}\right\rangle \varphi_{2}+\underbrace{\left(\left\langle x, \varphi_{1}\right\rangle-\left\langle x, \varphi_{1}\right\rangle\right)\left(\varphi_{1}-\varphi_{2}\right)}_{0},
$$

and rearrange it slightly to read

$$
x=\left\langle x, 2 \varphi_{1}\right\rangle \varphi_{1}+\left\langle x,\left(-\varphi_{1}+\varphi_{2}\right)\right\rangle \varphi_{2}+\left\langle x,-\varphi_{1}\right\rangle\left(\varphi_{1}-\varphi_{2}\right) .
$$

In the above, we can recognize $\left(-\varphi_{1}+\varphi_{2}\right)$ as $-\varphi_{3}$, and the vectors inside the inner products we will call

$$
\tilde{\varphi}_{1}=2 \varphi_{1}, \quad \tilde{\varphi}_{2}=-\varphi_{1}+\varphi_{2}, \quad \tilde{\varphi}_{3}=-\varphi_{1} .
$$

With this notation, we can rewrite the expansion as

$$
x=\left\langle x, \tilde{\varphi}_{1}\right\rangle \varphi_{1}+\left\langle x, \tilde{\varphi}_{2}\right\rangle \varphi_{2}+\left\langle x, \tilde{\varphi}_{3}\right\rangle \varphi_{3}=\sum_{i=1}^{3}\left\langle x, \tilde{\varphi}_{i}\right\rangle \varphi_{i},
$$


or, if we introduce matrix notation as before:

$$
\Phi=\left(\begin{array}{rrr}
1 & 0 & 1 \\
0 & 1 & -1
\end{array}\right), \quad \tilde{\Phi}=\left(\begin{array}{rrr}
2 & -1 & -1 \\
0 & 1 & 0
\end{array}\right)
$$

and

$$
x=\sum_{i=1}^{3}\left\langle x, \tilde{\varphi}_{i}\right\rangle \varphi_{i}=\Phi \tilde{\Phi}^{*} x .
$$

The only difference between the above expression and the one for general bases is that matrices $\Phi$ and $\tilde{\Phi}$ are now rectangular. Figure 3.1 shows this example pictorially.

We have thus shown that starting with an orthonormal basis and adding a vector, we obtained another expansion with three vectors. This expansion is reminiscent of the one for general biorthogonal bases we have seen earlier, except that the vectors involved in the expansion are now linearly dependent. This redundant set of vectors $\Phi=\left\{\varphi_{i}\right\}_{i \in I}$ is called a frame while $\tilde{\Phi}=\left\{\tilde{\varphi}_{i}\right\}_{i \in I}$ is called a dual frame of $\Phi=\left\{\varphi_{i}\right\}_{i \in I}$. As for biorthogonal bases, these two are interchangeable, and thus, $x=\Phi \tilde{\Phi}^{*} x=\tilde{\Phi} \Phi^{*} x$.

A Note of Caution: In many texts on frame theory, the frame change is usually denoted by $\Phi$, not $\tilde{\Phi}^{*}$. Given that $\Phi$ and $\tilde{\Phi}$ are interchangeable, we can use one or the other without risk of confusion. Since $\sum_{i \in I} X_{i} \varphi_{i}$ is really the expansion in terms of the basis/frame $\Phi$, we believe it is natural to use $\Phi$ on the reconstruction side and $\tilde{\Phi}^{*}$ on the decomposition side.

While adding a vector worked, we ended up with an expansion that does not look elegant, as it involves both the frame and its dual. Is it possible to have frames which would somehow mimic orthonormal bases? To do that, let us think for a moment what characterizes orthonormal bases. How about:

(1) Orthonormal bases are self dual?

(2) Orthonormal bases preserve the norm?

To answer these questions, we consider the simplest frame, aptly named Mercedes-Benz frame. 
Mercedes-Benz Frame: The Mercedes-Benz $(M B)$ frame $^{1}$ is arguably the most famous frame. It is a collection $\Phi$ of three vectors in $\mathbb{R}^{2}$, and is an excellent representative for many classes of frames. For example, the Mercedes-Benz frame is the simplest harmonic tight frame (introduced in Section 6.1).

Consider the version $\Phi_{\mathrm{PTF}}=\left\{\varphi_{1}, \varphi_{2}, \varphi_{3}\right\}$, where the frame has been scaled so that $\Phi_{\mathrm{PTF}} \Phi_{\mathrm{PTF}}^{*}=I^{2}$ :

$$
\Phi_{\mathrm{PTF}}^{*}=\left(\begin{array}{cc}
0 & \sqrt{2 / 3} \\
-1 / \sqrt{2} & -1 / \sqrt{6} \\
1 / \sqrt{2} & -1 / \sqrt{6}
\end{array}\right)
$$

and thus the expansion expression is

$$
x=\sum_{i=1}^{3}\left\langle x, \varphi_{i}\right\rangle \varphi_{i}=\Phi_{\mathrm{PTF}} \Phi_{\mathrm{PTF}}^{*} x,
$$

with the norm:

$$
\|X\|^{2}=\sum_{i=1}^{3}\left|\left\langle x, \varphi_{i}\right\rangle\right|^{2}=\|x\|^{2} .
$$

As $\Phi_{\mathrm{PTF}} \Phi_{\mathrm{PTF}}^{*}=I, \Phi_{\mathrm{PTF}}$ can represent any $x$ from $\mathbb{R}^{2}$ (real plane). Since in (3.3) the same set of vectors is used both for expansion and reconstruction, $\Phi_{\mathrm{PTF}}$ is self dual. We can think of the expansion in (3.3) as a generalization of an orthonormal basis except that the vectors are not linearly independent anymore. The frame of this type is called a tight frame (Parseval tight).

We can normalize the lengths of all the frame vectors to 1 , leading to the unit-norm version of this frame given as $\Phi_{\mathrm{UNTF}}=\left\{\varphi_{1}, \varphi_{2}, \varphi_{3}\right\}$ as in Figure 3.2:

$$
\Phi_{\mathrm{UNTF}}^{*}=\sqrt{\frac{3}{2}} \Phi_{\mathrm{PTF}}^{*}=\left(\begin{array}{cc}
0 & 1 \\
-\sqrt{3} / 2 & -1 / 2 \\
\sqrt{3} / 2 & -1 / 2
\end{array}\right)=\left(\begin{array}{c}
\varphi_{1}^{*} \\
\varphi_{2}^{*} \\
\varphi_{3}^{*}
\end{array}\right),
$$

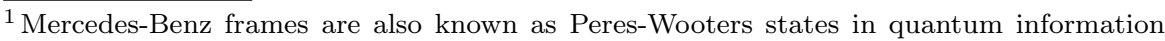
theory [148].

2 The subscript PTF stands for Parseval tight frame, which is a frame satisfying $\Phi_{\mathrm{PTF}} \Phi_{\mathrm{PTF}}^{*}=I$. We will elaborate on this class of frames later in the survey.
} 


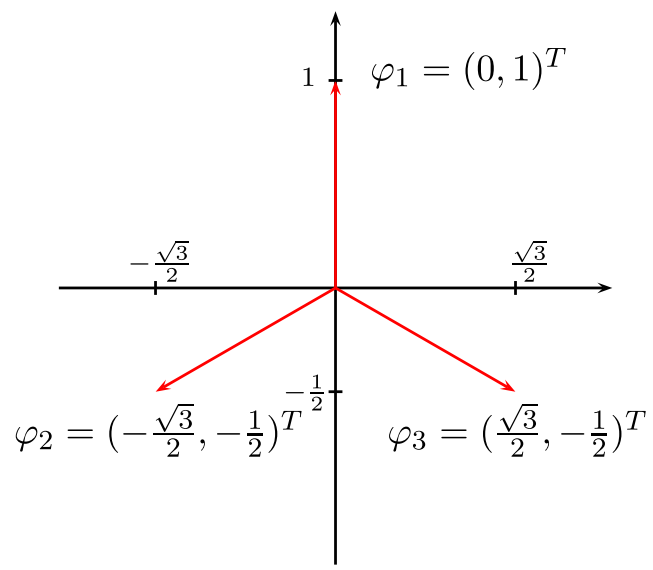

Fig. 3.2 Simplest unit-norm tight frame - Mercedes Benz frame. This is also an example of a harmonic tight frame from Section 6.1.

with the corresponding expansion:

$$
x=\frac{2}{3} \sum_{i=1}^{3}\left\langle x, \varphi_{i}\right\rangle \varphi_{i}=\frac{2}{3} \Phi_{\mathrm{UNTF}} \Phi_{\mathrm{UNTF}}^{*} x,
$$

and the norm:

$$
\|X\|^{2}=\sum_{i=1}^{3}\left|\left\langle x, \varphi_{i}\right\rangle\right|^{2}=\frac{3}{2}\|x\|^{2} .
$$

We can compare the expansion into an orthonormal basis (2.1) with the expansion into a unit-norm version of the Mercedes-Benz frame (3.3), and see that the frame version has an extra scaling of $2 / 3$. When the frame is tight and all the vectors have unit norm as in this case, the inverse of this scaling factor denotes the redundancy of the system: we have $3 / 2$ or $50 \%$ more vectors than needed to represent any vector in $\mathbb{R}^{2}$. Note that, in general, relating the frame bounds to the redundancy factor does not seem to be possible for frames that are not tight.

This discussion took care of the first question, whether we can have a self-dual frame. To check the question about norms, we compute the sum of the squared transform coefficients as in (3.4), and see that, indeed, this frame preserves the norm. To make the comparison to orthonormal bases fair, again we take the unit-norm version of the 
frame and compute the sum of the squared transform coefficients as in (3.7). Now there is extra scaling of $3 / 2$; this is fairly intuitive, as in the transform domain, where we have more coefficients than we started with, the energy is $3 / 2$ times higher than in the original domain.

Thus, the tight frame we constructed is very similar to an orthonormal basis, with a linearly dependent set of vectors. Actually, tight frames are redundant sets of vectors closest to orthonormal bases (we will make this statement precise in Section 4.2).

One more interesting tidbit about this particular frame; note how all its vectors have the same norm. This is not necessary for tightness, but when true, the frame is called an equal-norm tight frame.

\subsection{General Frames}

In the last section, we introduced frames through examples and developed some intuition. We now discuss frames more generally and examine a few of their properties.

A family $\Phi=\left\{\varphi_{i}\right\}_{i \in I}$ in a Hilbert space $\mathbb{H}$ is called a frame if there exist two constants $0<A \leq B<\infty$, such that for all $x$ in $\mathbb{H}$,

$$
A\|x\|^{2} \leq \sum_{i \in I}\left|\left\langle x, \varphi_{i}\right\rangle\right|^{2} \leq B\|x\|^{2} .
$$

$A, B$ are called frame bounds. Tight frames are frames with equal frame bounds, that is, $A=B$. Equal-norm frames are those frames where all the elements have the same norm, $\left\|\varphi_{i}\right\|=\left\|\varphi_{j}\right\|$, for $i, j \in I$. Unit-norm frames are those frames where all the elements have norm $1,\left\|\varphi_{i}\right\|=1$, for $i \in I$. A-tight frames are tight frames with frame bound $A$. The special case of 1-tight frames are usually called Parseval tight frames. These various classes of frames are illustrated in Figure 3.3.

The frame bounds are intimately related to the issues of stable reconstruction. First, the operator mapping $x \in \ell^{2}(\mathbb{Z})$ into its transform coefficients $\left|\left\langle x, \varphi_{i}\right\rangle\right|$ has to be bounded, that is, $\sum_{i \in I}\left|\left\langle x, \varphi_{i}\right\rangle\right|^{2}$ has to be finite, achieved by the bound from above. Second, no $x$ with $\|x\|>0$ should be mapped to 0 , achieved by the bound from below. Thus, a numerically stable reconstruction of any $x$ from its transform coefficients is possible only if (3.8) is satisfied. The closer the frame 


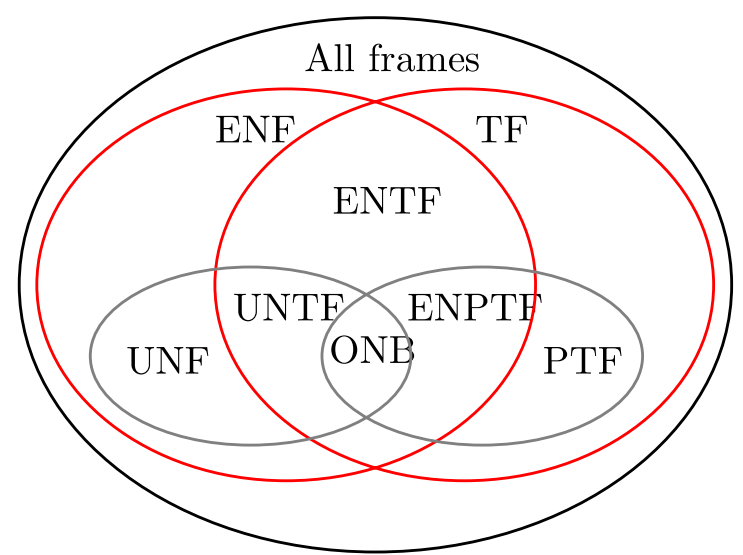

Fig. 3.3 Frames at a glance. ENF: Equal-norm frames, TF: Tight frames, ENTF: Equalnorm tight frames, UNF: Unit-norm frames, PTF: Parseval tight frames, UNTF: Unit-norm tight frames, ENPTF: Equal-norm Parseval tight frames, ONB: Orthonormal bases.

bounds are, the faster and numerically better behaved reconstruction we have.

Any finite set of vectors that spans the space is a frame. This can be seen from (3.8). Since $\sum_{i \in I}\left|\left\langle x, \varphi_{i}\right\rangle\right|^{2}=\tilde{\Phi} \tilde{\Phi}^{*}\|x\|^{2}$, that sum is bounded from below and above by the smallest and largest eigenvalue of $\tilde{\Phi} \tilde{\Phi}^{*}$, respectively, multiplying $\|x\|^{2}$. In the example given in (3.1), $A \simeq 0.8$ and $B \simeq 6.2$.

\subsubsection{Frame Operators}

The analysis operator $\Phi^{*}$ maps the Hilbert space $\mathbb{H}$ into $\ell^{2}(I)^{3}$ :

$$
X_{i}=\left(\Phi^{*} x\right)_{i}=\left\langle x, \varphi_{i}\right\rangle, \quad i \in I
$$

and, from (3.8), satisfies $^{4}$ :

$$
A I \leq \Phi \Phi^{*} \leq B I
$$

\footnotetext{
${ }^{3}$ The fact that $\left(\Phi^{*} x\right)_{i} \in \ell^{2}(I)$ comes from (3.8).

${ }^{4}$ The inequality $S \leq U$ for matrices $S, U$ means that $U-S$ is nonnegative definite.
} 
As a matrix, the analysis operator $\Phi^{*}$ has rows which are the Hermitian-transposed frame vectors $\varphi_{i}^{*}$ :

$$
\Phi^{*}=\left(\begin{array}{cccc}
\varphi_{11}^{*} & \cdots & \varphi_{1 n}^{*} & \cdots \\
\varphi_{21}^{*} & \cdots & \varphi_{2 n}^{*} & \cdots \\
\vdots & \ddots & \ddots & \cdots \\
\varphi_{m 1}^{*} & \cdots & \varphi_{m n}^{*} & \cdots \\
\vdots & \vdots & \vdots & \ddots
\end{array}\right)
$$

When $\mathbb{H}=\mathbb{R}^{n}, \mathbb{C}^{n}$, the above is an $m \times n$ matrix. When $\mathbb{H}=\ell^{2}(\mathbb{Z})$, it is an infinite matrix.

The following two operators play an important role in frame theory: the frame operator, defined as $S=\Phi \Phi^{*}$, and the Grammian ${ }^{5}$ defined as $G=\Phi^{*} \Phi$. The frame operator $S$ is bounded, invertible, self-adjoint, and positive [51].

The canonical dual frame of $\Phi$ is a frame defined as $\tilde{\Phi}=\left\{\tilde{\varphi}_{i}\right\}_{i \in I}=$ $\left\{S^{-1} \varphi_{i}\right\}_{i \in I}$, where

$$
\tilde{\varphi}_{i}=S^{-1} \varphi_{i}, \quad i \in I
$$

Noting that $\tilde{\varphi}_{i}^{*}=\varphi_{i}^{*} S^{-1}$ and stacking $\tilde{\varphi}_{1}^{*}, \tilde{\varphi}_{2}^{*}, \ldots$, in a matrix, the analysis frame operator associated with $\tilde{\Phi}$ is

$$
\tilde{\Phi}^{*}=\Phi^{*} S^{-1},
$$

while its frame operator is $S^{-1}$, with $B^{-1}$ and $A^{-1}$ its frame bounds [51]. Since

$$
\Phi \tilde{\Phi}^{*}=\underbrace{\Phi \Phi^{*}}_{S} S^{-1}=I
$$

then

$$
x=\sum_{i \in I}\left\langle x, \tilde{\varphi}_{i}\right\rangle \varphi_{i}=\Phi \tilde{\Phi}^{*} x=\tilde{\Phi} \Phi^{*} x=\sum_{i \in I}\left\langle x, \varphi_{i}\right\rangle \tilde{\varphi}_{i} .
$$

\footnotetext{
${ }^{5}$ Both Grammian and Gramian are used.
} 
To reconstruct $x$ from its frame coefficients, we need only to compute $\tilde{\varphi}_{i}$ via (the proof is given in [64]):

$$
\tilde{\varphi}_{i}=\frac{2}{A+B} \sum_{i=0}^{\infty}\left(I-\frac{2}{A+B} S\right)^{i} \varphi_{i} .
$$

When $\frac{B}{A}-1 \ll 1$, from (3.9), $S$ is close to $\frac{A+B}{2} I$ and $\left(I-\frac{2}{A+B} S\right)$ is small, leading to fast convergence. When the frame is tight, $A=B$, $\tilde{\varphi}_{i}=\frac{1}{A} \varphi_{i}$, and inversion is not necessary.

Note that the dual operator is not unique, as any left-inverse of the analysis operator can be used for reconstructing $x$. In particular, classical results in [91] provide a complete parametrization of all left-inverses. The canonical dual frame corresponds to the minimum norm reconstruction. Sometimes, the canonical dual is undesirable, for example Daubechies presents a case in [63] where the canonical dual of a wavelet frame does not have the wavelet structure. In [52], Christensen and Eldar explore other duals.

To summarize what we have done until now:

(1) We represented our signal in another domain to more easily extract its salient characteristics. We did that in a redundant fashion.

(2) Given a pair of dual frames $(\Phi, \tilde{\Phi})$, the coordinates of our signal in the new domain (that is, with respect to the new frame) are given by

$$
X=\tilde{\Phi}^{*} x .
$$

This is called the analysis or decomposition expression. In $\mathbb{R}^{n}, \mathbb{C}^{n}$, with $m>n$ frame vectors, $\tilde{\Phi}$ is a rectangular $n \times m$ matrix describing the frame change and it contains dual frame vectors as its columns, while $X$ collects all the transform coefficients together.

(3) The synthesis, or reconstruction is given by

$$
x=\Phi X \text {. }
$$

In $\mathbb{R}^{n}, \mathbb{C}^{n}$, with $m>n$ frame vectors, $\Phi$ is again a rectangular $n \times m$ matrix, and it contains frame vectors as its columns. 


\section{Frame Definitions and Properties}

(4) If the expansion is into a general frame, then

$$
\Phi \tilde{\Phi}^{*}=I
$$

When manipulating frame expressions, facts given below (some frame specific and the others valid for general matrices) often come in handy:

(1) For any matrix $\Phi^{*}$ with rows $\varphi_{i}^{*}$,

$$
S=\Phi \Phi^{*}=\sum_{i \in I} \varphi_{i} \varphi_{i}^{*}
$$

(2) If $S$ is a frame operator, then

$$
\begin{aligned}
S x & =\Phi \Phi^{*} x=\sum_{i \in I}\left\langle x, \varphi_{i}\right\rangle \varphi_{i} \\
\langle x, S x\rangle & =\left\langle x, \Phi \Phi^{*} x\right\rangle=\left\langle\Phi^{*} x, \Phi^{*} x\right\rangle \\
& =\left\|\Phi^{*} x\right\|^{2}=\sum_{i \in I}\left|\left\langle x, \varphi_{i}\right\rangle\right|^{2}, \\
\sum_{i \in I}\left\langle\varphi_{i}, S \varphi_{i}\right\rangle & =\sum_{i \in I}\left\langle\Phi^{*} \varphi_{i}, \Phi^{*} \varphi_{i}\right\rangle=\sum_{i, j \in I}\left|\left\langle\varphi_{i}, \varphi_{j}\right\rangle\right|^{2} .
\end{aligned}
$$

(3) From (3.8), we have that

$$
A I \leq S \leq B I
$$

as well as

$$
B^{-1} I \leq S^{-1} \leq A^{-1} I .
$$

(4) We say that two frames $\Phi$ and $\Psi$ for $\mathbb{H}$ are equivalent, if there exists a bounded linear bijection ${ }^{6} L$ on $\mathbb{H}$ for which $L \varphi_{i}=\psi_{i}$ for $i \in I$. Two frames $\Phi$ and $\Psi$ are unitarily equivalent if $L$ can be chosen to be a unitary operator. Any $A$-tight frame is equivalent to a Parseval tight frame as $\varphi_{\mathrm{PTF}}=(1 / \sqrt{A}) \varphi_{A-\mathrm{TF}}$.

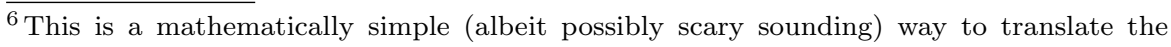
notion of "invertibility" to an infinite-dimensional Hilbert space.
} 
(5) In finite dimensions, the nonzero eigenvalues $\left\{\lambda_{i}\right\}_{i \in I}$, of $S=$ $\Phi \Phi^{*}$ and $G=\Phi^{*} \Phi$ are the same:

$$
\operatorname{tr}\left(\Phi \Phi^{*}\right)=\operatorname{tr}\left(\Phi^{*} \Phi\right)
$$

(6) A $\Phi^{*}$ matrix of a tight frame has orthonormal columns. In finite dimensions, this is equivalent to the Naimark theorem (see Section 4.1), which says that every tight frame is obtained by projecting an orthonormal basis from a larger space.

\subsection{Tight Frames}

From (3.8), in a tight frame (that is, when $A=B$ ), we have

$$
\sum_{i \in I}\left|\left\langle x, \varphi_{i}\right\rangle\right|^{2}=A\|x\|^{2} .
$$

By pulling $1 / A$ into the sum, this is equivalent to:

$$
\sum_{i \in I}\left|\left\langle\frac{1}{\sqrt{A}} x, \varphi_{i}\right\rangle\right|^{2}=\|x\|^{2},
$$

that is, the family $\Phi=\left\{(1 / \sqrt{A}) \varphi_{i}\right\}_{i \in I}$ is a 1-tight frame. In other words, any tight frame can be rescaled to be a tight frame with frame bound 1 - a Parseval tight frame, for which $\Phi \Phi^{*}=I$. With $A=1$, the above looks similar to (2.4), Parseval's equality, thus the name Parseval tight frame.

In an A-tight frame, $x \in \mathbb{H}$ is expanded as:

$$
x=\frac{1}{A} \sum_{i \in I}\left\langle x, \varphi_{i}\right\rangle \varphi_{i}
$$

While this last equation resembles the expansion formula in the case of an orthonormal basis as in (2.1) and (2.2) (except for the factor 1/A), a frame does not constitute an orthonormal basis in general. In particular, vectors may be linearly dependent and thus not form a basis. If all the vectors in a tight frame have unit norm, then the constant $A$ gives the redundancy ratio. For example, $A=2$ means there are twice as many 
vectors than needed to cover the space. For the Mercedes-Benz frame we discussed earlier, redundancy is $3 / 2$, that is, we have $3 / 2$ times more vectors than needed to represent vectors in a two-dimensional space. Note that if $A=B=1$ (Parseval tight frame), and $\left\|\varphi_{i}\right\|=1$ for all $i$, then $\Phi=\left\{\varphi_{i}\right\}_{i \in I}$ is an orthonormal basis (see Figure 3.3).

Because of the linear dependence which exists among the vectors used in the expansion, the expansion is no longer unique. Consider $\Phi=\left\{\varphi_{i}\right\}_{i \in I}$, where $\sum_{i \in I} \alpha_{i} \varphi_{i}=0$ (where not all $\alpha_{i}$ 's are zero because of linear dependence). If $x$ can be written as $x=\sum_{i \in I} X_{i} \varphi_{i}$, then one can add $\alpha_{i}$ to each $X_{i}$ without changing the decomposition. The expansion (3.16) is unique in the sense that it minimizes the norm of the expansion among all valid expansions. Similarly, for general frames, there exists a unique canonical dual frame, which we discussed earlier in this section (in the tight frame case, the frame and its canonical dual are equal).

To summarize, when the expansion is into a tight frame, then

$$
\tilde{\Phi}=\Phi, \quad \text { and } \quad \Phi \Phi^{*}=A I_{n \times n} .
$$

Note that, unlike for bases, $\Phi^{*} \Phi$ is not necessarily an identity. 
We now consider finite-dimensional frames, that is, when $\mathbb{H}=\mathbb{R}^{n}, \mathbb{C}^{n}$, and examine a few of their properties.

For example, for an equal-norm tight frame with norm- $a$ vectors, since $S=\Phi \Phi^{*}=A I_{n \times n}$,

$$
\operatorname{tr}(S)=\sum_{j=1}^{n} \lambda_{j}=n A,
$$

where $\lambda_{j}$ are the eigenvalues of $S=\Phi \Phi^{*}$. On the other hand, because of $(3.13)$

$$
\operatorname{tr}(S)=\operatorname{tr}(G)=\sum_{i=1}^{m}\left\|\varphi_{i}\right\|^{2}=m a^{2} .
$$

Combining (4.1) and (4.2), we get

$$
A=\frac{m}{n} a^{2} .
$$

Then, for a unit-norm tight frame, that is, when $a=1$, (4.3) yields the redundancy ratio:

$$
A=\frac{m}{n} .
$$

Recall that for the Mercedes-Benz frame, $A=3 / 2$. 
These, and other trace identities for all frame classes are given in Table A.3.

\subsection{Naimark Theorem}

The following theorem tells us that every Parseval tight frame can be realized as a projection of an orthonormal basis from a larger space. ${ }^{1}$ In this survey, we consider only the finite-dimensional instantiation of the theorem (one possible proof of which is given in [80]).

Theorem 4.1 ([2], [99]). A set $\Phi=\left\{\varphi_{i}\right\}_{i \in I}$ in a Hilbert space $\mathbb{H}$ is a Parseval tight frame for $\mathbb{H}$ if and only if there is a larger Hilbert space $\mathbb{K}, \mathbb{H} \subset \mathbb{K}$, and an orthonormal basis $\left\{e_{i}\right\}_{i \in I}$ for $\mathbb{K}$ so that the orthogonal projection $P$ of $\mathbb{K}$ onto $\mathbb{H}$ satisfies: $P e_{i}=\varphi_{i}$, for all $i \in I$.

While the above theorem specifies how all tight frames are obtained, the same is true in general, that is, any frame can be obtained by projecting a biorthogonal basis from a larger space [99] (we are talking here about finite dimensions only). We will call this process seeding and will say that a frame $\Phi$ is obtained by seeding from a basis $\Psi$ by deleting a suitable set of columns of $\Psi$ [139]. We denote this as

$$
\Phi^{*}=\Psi[J]
$$

where $J \subset\{1, \ldots, m\}$ is the index set of the retained columns.

We can now reinterpret the Parseval tight frame identity $\Phi \Phi^{*}=I$ : It says that the columns of $\Phi^{*}$ are orthonormal. In view of the above theorem, this makes a lot of sense as that frame was obtained by deleting columns from an orthonormal basis from a larger space.

\footnotetext{
${ }^{1}$ The theorem has been rediscovered by several people in the past decade: The first author heard it from Daubechies in the mid-90's. Han and Larson rediscovered it in [99]; they came up with the idea that a frame could be obtained by compressing a basis in a larger space and that the process is reversible. Finally, it was Šoljanin [151] who pointed out to the first author that this is, in fact, Naimark theorem, which has been widely known in operator algebra and used in quantum information theory.
} 
Example 4.1 (Mercedes-Benz frame cont'd). For example, the Parseval tight frame version of the Mercedes-Benz frame (3.2) can be obtained by projecting an orthonormal basis from a three-dimensional space:

$$
\Psi=\left(\begin{array}{ccc}
0 & \sqrt{2 / 3} & 1 / \sqrt{3} \\
-1 / \sqrt{2} & -1 / \sqrt{6} & 1 / \sqrt{3} \\
1 / \sqrt{2} & -1 / \sqrt{6} & 1 / \sqrt{3}
\end{array}\right)
$$

using the following projection operator $P$ :

$$
P=\frac{1}{\sqrt{3}}\left(\begin{array}{ccc}
2 / 3 & -1 / 3 & -1 / 3 \\
-1 / 3 & 2 / 3 & -1 / 3 \\
-1 / 3 & -1 / 3 & 2 / 3
\end{array}\right),
$$

that is, the Mercedes-Benz frame seen as a collection of vectors in the three-dimensional space is $\Phi_{3 \mathrm{D}}=P \Psi$. The projection operator essentially "deletes" the last column of $\Psi$ to create the frame operator $\Phi^{*}$. For equal-norm tight frames such as Mercedes-Benz, the connection to an orthonormal basis in a higher dimension is similar to the relationship of a regular simplex signal constellation in dimension $n$ to the orthogonal constellation in dimension $(n+1)$ [174].

\subsection{What Can Coulomb Teach Us?}

As the orthonormal bases have specific characteristics highly prized among bases, the same distinction belongs to tight frames among all frames. As such, they have been studied extensively, but only recently have Benedetto and Fickus [13] formally shown why tight frames and orthonormal bases indeed belong together. In their work, they characterized all unit-norm tight frames, while in [40], the authors did the same for nonequal norm tight frames.

To characterize unit-norm tight frames, as a starting point, the authors looked at harmonic tight frames (we will introduce those in Section 6.1 ), obtained by taking $m$ th roots of unity in $\mathbb{C}^{n}$. These lead to 
regular arrangement of points on a circle (an example is the MercedesBenz frame from Figure 3.2). Trying to generalize the notion of geometric regularity to three dimensions, they looked at vertices of regular polyhedra but came up short as there are only five such Platonic solids. Considering other sets of high symmetry such as the "soccer ball" (a truncated icosahedron), they found that all these proved to be unitnorm tight frames.

As the geometric intuition could lead them only so far, the authors in [13] refocused their attention on the equidistribution properties of these highly symmetric objects and thought of the notion of equilibrium. To formalize that notion, they turned to classical physics and considered the example of $m$ electrons on a conductive spherical shell. In the absence of external forces, electrons move according to the Coulomb force law until they reach the state of minimum potential energy (though that minimum might only be a local minimum leading to an unstable equilibrium). The intuition developed through this example led them to the final result.

The authors tried to replicate the physical world for the simplest unit-norm tight frames - orthonormal bases, and thought of what kind of equilibrium they possessed. Clearly, whichever "force" acts on the vectors in an orthonormal basis, it tries to promote orthogonality. For example, the Coulomb force would not keep the orthonormal basis in a state of equilibrium. (Think $n=2$, the Coulomb force would position the two vectors to be colinear of opposite sign.) Thus, the authors set to find another such force - the orthogonality-promoting one. This force should be repulsive if vectors form an acute angle, while it should be attractive if they form an obtuse angle. Since points are restricted to move only on the circle (unit-norm constraint), one can consider only the tangential component of the force. When vectors do not all have equal norm, $\left\|\varphi_{i}\right\|=a_{i}$, for $i \in I$, the authors in [13] propose a definition of the frame force $\mathrm{FF}$ to encompass the whole space:

$$
\begin{aligned}
\mathrm{FF}\left(\varphi_{i}, \varphi_{j}\right) & =2\left\langle\varphi_{i}, \varphi_{j}\right\rangle\left(\varphi_{i}-\varphi_{j}\right) \\
& =\left(a_{i}^{2}+a_{j}^{2}-\left\|\varphi_{i}-\varphi_{j}\right\|^{2}\right)\left(\varphi_{i}-\varphi_{j}\right) .
\end{aligned}
$$


Following the physical trail, one can now define the potential between two points as:

$$
P\left(\varphi_{i}, \varphi_{j}\right)=p\left(\left\|\varphi_{i}-\varphi_{j}\right\|\right) .
$$

This is found by using $p^{\prime}(x)=-x f(x)$, where $f(x)$ is the magnitude of the frame force and $p(x)$ is obtained by integrating the above and evaluating at $\left\|\varphi_{i}-\varphi_{j}\right\|^{2}$. After some manipulations, the result is

$$
P\left(\varphi_{i}, \varphi_{j}\right)=\left\langle\varphi_{i}, \varphi_{j}\right\rangle^{2}-\frac{1}{4}\left(a_{i}^{2}+a_{j}^{2}\right)^{2} .
$$

Then, the total potential contained within a sequence is

$$
T P\left(\Phi=\left\{\varphi_{i}\right\}_{i \in I}\right)=\sum_{i, j \in I, i \neq j}\left|\left\langle\varphi_{i}, \varphi_{j}\right\rangle\right|^{2}-\frac{1}{4} \sum_{i, j}\left(a_{i}^{2}+a_{j}^{2}\right)^{2} .
$$

For details of derivations, we refer the reader to [13].

Physically, we can interpret the total potential as follows: Given two sequences of points, the difference in potentials between these two sequences is the energy needed to move the points from one configuration to the other. As potential energy is defined in terms of differences, it is unique up to additive constants and thus we can neglect the constants and add the diagonal terms to obtain the final expression for the frame potential:

$$
\operatorname{FP}\left(\Phi=\left\{\varphi_{i}\right\}_{i \in I}\right)=\sum_{i, j \in I}\left|\left\langle\varphi_{i}, \varphi_{j}\right\rangle\right|^{2} .
$$

Thus, what we are looking for are those sequences in equilibrium under the frame force, and these will be minimizers of the frame potential.

For unit-norm tight frames, Benedetto and Fickus discovered the following:

Theorem 4.2 ([13]). Given $\Phi=\left\{\varphi_{i}\right\}_{i=1}^{m}$, with $\varphi_{i} \in \mathbb{H}^{n}$, consider the frame potential given in (4.6). Then:

(1) Every local minimizer of the frame potential is also a global minimizer.

(2) If $m \leq n$, the minimum value of the frame potential is

$$
F P=n,
$$


and the minimizers are precisely the orthonormal sequences in $\mathbb{R}^{n}$.

(3) If $m \geq n$, the minimum value of the frame potential is

$$
F P=\frac{m^{2}}{n},
$$

and the minimizers are precisely the unit-norm tight frames for $\mathbb{R}^{n}$.

The above result tells us a few things:

(1) Minimizing the frame potential amounts to finding sequences whose elements are "as orthogonal" to each other as possible.

(2) Unit-norm tight frames are a natural extension of orthonormal bases, that is, the theorem formalizes the intuitive notion that unit-norm tight frames are a generalization of orthonormal bases.

(3) Both orthonormal bases and unit-norm tight frames are results of the minimization of the frame potential, with different parameters (number of elements equal/larger than the dimension of the space).

What happens if points live on different spheres, $\varphi_{i}=a_{i}$ (vectors are not of equal norm)? Again, we can try to minimize the frame potential. Since now points live on spheres of different radii, it is intuitive that stronger points (with a larger norm) will be able to be "more orthogonal" than the weaker ones. If the strongest point is strong enough, it grabs a dimension to itself and leaves the others to squabble over what is left. We start all over with the second one and continue until those points left have to share. This is governed by the fundamental inequality:

$$
\max _{i \in I} a_{i}^{2} \leq \frac{1}{n} \sum_{i \in I} a_{i}^{2}
$$

where $a_{i}=\left|\varphi_{i}\right|$, which says that if no point is stronger than the rest they immediately have to share, leading to tight frames. In other words, 
when $m$ points in an $n$-dimensional space are in equilibrium, we can divide those points into two sets. (a) Those "stronger" than the rest. These $\left(i_{0}-1\right)$ points get a dimension each and are thus orthogonal to each other. (b) Those "weaker" than the rest. These points get the rest of the $\left(n-i_{0}+1\right)$ dimensions and form a tight frame for their span. If no point is the "strongest," they all have to share the space leading to a tight frame, as per the fundamental inequality. This discussion is summarized in the theorem below:

Theorem 4.3 ([40]). Given a sequence $\left\{a_{i}=\left\|\varphi_{i}\right\|\right\}_{i=1}^{m}$ in $\mathbb{R}$, such that $a_{1} \geq \cdots \geq a_{m} \geq 0$, and any $n \leq m$, let $i_{0}$ denote the smallest index $i$ for which

$$
(n-i) a_{i}^{2} \leq \sum_{j=i+1}^{m} a_{j}^{2}
$$

holds. Then, any local minimizer of the frame potential is of the form:

$$
\Phi=\left\{\varphi_{i}\right\}_{i=1}^{m}=\left\{\varphi_{i}\right\}_{i=1}^{i_{0}-1} \cup\left\{\varphi_{i}\right\}_{i=i_{0}}^{m}
$$

where $\Phi_{o}=\left\{\varphi_{i}\right\}_{i=1}^{i_{0}-1}$ is an orthogonal set and $\Phi_{f}=\left\{\varphi_{i}\right\}_{i=i_{0}}^{m}$ forms a tight frame for the orthogonal complement of the span of $\Phi_{o}$.

The immediate corollary is the fundamental inequality (4.7).

The frame potential defined in (4.6) proved immediately useful. For example, it was used in [41] to show how to packetize coefficients in transmission with erasures to minimize the error of reconstruction. Recently, Benedetto and Kebo [14] used the frame force to solve a quantum detection problem, where the goal is to construct a tight frame that minimizes an error term, interpreted in quantum mechanics as the probability of a detection error. A decade before [13], Massey and Mittelholzer [131] used the frame potential (albeit not calling it the frame potential) as the total user interference in code-division multiple access (CDMA) systems. Minimizing that interference lead to the spreading sequences (of length $n$ ) being a tight frame (minimum of the Welch bound). This is discussed in more detail in Section 7.6. 
34 Finite-Dimensional Frames

\subsection{Design Constraints: What Might We Ask of a Frame?}

When designing a frame, particularly if we have a specific application in mind, it is useful to list potential requirements we might impose on our frame.

(1) Tightness: This is a very common requirement. Typically, tightness is imposed when we do need to reconstruct. Since tight frames do not require inversion of matrices, they seem a natural choice.

(2) Equal norm: In the real world, the squared norm of a vector is usually associated with power. Thus, in situations where equal-power signals are desirable, equal norm is a must.

(3) Maximum robustness: We call a frame maximally robust to erasures, if every $n \times n$ submatrix of $\Phi^{*}$ is invertible. This requirement arose in using frames for robust transmission [92] and will be discussed in more detail in Section 7.4.

(4) Equiangularity: This is a geometrically intuitive requirement. We ask for angles between any two vectors to be the same. There are many more (tight) frames than those which are equiangular, so this leads to a very particular class of frames. These are discussed in more detail in Section 6.2.

(5) Symmetry: Symmetries in a frame are typically connected to its geometric configuration. Harmonic and equiangular frames are good examples. See the work of Vale and Waldron [164] for details.

Invariance of Frame Properties: When designing frames, it is useful to know which transformations will not destroy properties our frame already possesses. For that reason, we list below a number of frame invariance properties [139]. Let $\Phi$ be a frame. In all matrix products below, we assume the sizes to be compatible.

(1) $V \Phi U$ is a frame for any invertible matrices $U, V$.

(2) If $\Phi$ is tight frame/unit-norm tight frame, then $a V \Phi U$ is tight frame/unit-norm tight frame for any unitary matrices $U, V$ and $a \neq 0$. 
(3) If $\Phi$ is equal-norm, then $a D \Phi U$ is equal-norm for any diagonal unitary matrix $D$, unitary matrix $U$, and $a \neq 0$.

(4) If $\Phi$ is maximally robust, then $D \Phi U$ is maximally robust for any invertible diagonal matrix $D$ and any invertible matrix $U$.

(5) If $\Phi$ is unit-norm tight frame and maximally robust, then $D \Phi U$ is unit-norm tight frame and maximally robust for any unitary diagonal matrix $D$ and any unitary matrix $U$. 


\section{Infinite-Dimensional Frames via Filter Banks}

We now consider the only infinite-dimensional class of frames discussed in this survey, those implemented by filter banks, the reason being that these are frames used in applications and our only link to the real world.

The vectors (signals) live in the infinite-dimensional Hilbert space $\mathbb{H}=\ell^{2}(\mathbb{Z})$. An in-depth treatment of filter banks is given in [163], while a more expansion-oriented approach is followed in $[166,167]$. We first start by looking at filter bank implementations of bases as these easily extend to frames under certain conditions. We also study the cases that lead to important types of transforms.

\subsection{Bases via Filter Banks}

As we have done earlier in the survey, we will first examine how filter banks implement bases, and then move on to frames.

We have seen that we want to find representations or matrices $\Phi$ and $\tilde{\Phi}$ such that $\Phi \tilde{\Phi}^{*}=I$. As of now, we have presented a generic matrix $\Phi$, but how do we choose it? Of course, we want it to have some structure and lead to efficient representations of signals. Since now we are dealing with infinite-dimensional matrices, a possibly difficult task. 
Example 5.1. Given are $\varphi_{0}=(\ldots, 0,1,1,0, \ldots)^{T} / \sqrt{2}$, and $\varphi_{1}=$ $(\ldots, 0,1,-1,0, \ldots)^{T} / \sqrt{2}$. These vectors form a basis for their span, that is, they can represent any two-dimensional vector, but not any vector in $\ell^{2}(\mathbb{Z})$. Now, define $\tau^{i}$ as a shift by $i$, that is, if $x=\left(\ldots, x_{-1}, x_{0}, x_{1}, \ldots\right)^{T}$ $\in \ell^{2}(\mathbb{Z})$, then $\tau^{i} x=\left(\ldots, x_{-i-1}, x_{-i}, x_{-i+1}, \ldots\right)^{T}$ is its shifted version by $i$. Let us form the following matrix ${ }^{1}$ :

$$
\Phi^{*}=\left(\begin{array}{c}
\vdots \\
\left(\tau^{-2} \varphi_{0}\right)^{*} \\
\left(\tau^{-2} \varphi_{1}\right)^{*} \\
\left(\varphi_{0}\right)^{*} \\
\left(\varphi_{1}\right)^{*} \\
\left(\tau^{2} \varphi_{0}\right)^{*} \\
\left(\tau^{2} \varphi_{1}\right)^{*} \\
\vdots
\end{array}\right)
$$

that is, the columns of $\Phi$ are the two vectors $\varphi_{0}, \varphi_{1}$ and all their even shifts, leading to a block-diagonal $\Phi^{*}$ :

$$
\Phi^{*}=\frac{1}{\sqrt{2}}\left(\begin{array}{cccccccc}
\ddots & \vdots & \vdots & \vdots & \vdots & \vdots & \vdots & \\
\cdots & 1 & 1 & 0 & 0 & 0 & 0 & \cdots \\
\cdots & 1 & -1 & 0 & 0 & 0 & 0 & \cdots \\
\cdots & 0 & 0 & 1 & 1 & 0 & 0 & \cdots \\
\cdots & 0 & 0 & 1 & -1 & 0 & 0 & \cdots \\
\cdots & 0 & 0 & 0 & 0 & 1 & 1 & \cdots \\
\cdots & 0 & 0 & 0 & 0 & 1 & -1 & \cdots \\
& \vdots & \vdots & \vdots & \vdots & \vdots & \vdots & \ddots
\end{array}\right) .
$$

If we denote by

$$
\Phi_{0}^{*}=\frac{1}{\sqrt{2}}\left(\begin{array}{cc}
1 & 1 \\
1 & -1
\end{array}\right)
$$

\footnotetext{
${ }^{1}$ The "boxing" of the origin serves as a reference point when dealing with infinite vectors/matrices.
} 
then, $\Phi$ can be written as:

$$
\Phi^{*}=\left(\begin{array}{llllll}
\ddots & & & & \\
& \Phi_{0}^{*} & & & \\
& & \Phi_{0}^{*} & & \\
& & & \Phi_{0}^{*} & \\
& & & & \ddots
\end{array}\right) .
$$

This is known as the Haar transform and is an example of a block transform. The matrix $\Phi$ above is unitary and corresponds to an orthonormal basis expansion, $\Phi=\left\{\varphi_{2 i}, \varphi_{2 i+1}\right\}_{i \in \mathbb{Z}}=\left\{\tau^{2 i} \varphi_{0}, \tau^{2 i} \varphi_{1}\right\}_{i \in \mathbb{Z}}$. Therefore, any $x \in \ell^{2}(\mathbb{Z})$ can be represented using the Haar orthonormal basis as:

$$
x=\Phi \Phi^{*} x=\sum_{i \in \mathbb{Z}}\left\langle x, \varphi_{i}\right\rangle \varphi_{i}
$$

and can be implemented using the two-channel filter bank shown in Figure 5.1. The decomposition is implemented using the analysis filter bank, while the reconstruction is implemented using the synthesis filter bank (we will make this more precise shortly).

In general, in such a filter bank, one branch is a lowpass channel that captures the coarse representation of the input signal and the other branch is a highpass channel that captures a complementary, detailed representation. The input into the filter bank is a squaresummable infinite sequence $x \in \ell^{2}(\mathbb{Z})$. Assuming that the filter length

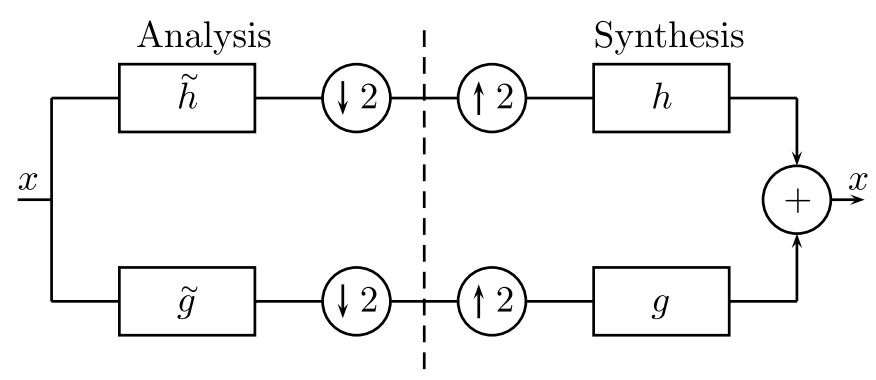

Fig. 5.1 Two-channel filter bank with downsampling by 2 . 
$l=2$, the two analysis filters act on 2 samples at a time and then, due to downsampling by 2 , the same filters act on the following 2 samples. In other words, there is no overlap. On the synthesis side, the reverse is true. This is an example of a block transform. Iterating this block (the two-channel filter bank) on either channel or both leads to various signal transforms, each of which is adapted to a class of signals with different energy concentrations in time and in frequency (this is usually referred to as "tiling of the time-frequency plane").

So how exactly is the filter bank related to the matrix $\Phi$ ? In our discussion above and the Haar example, we assumed that the filter length is equal to the shift. This is not true in general, and now, we lift that restriction and allow filters to be of arbitrary length $l$ (without loss of generality, we will assume that filters are causal, that is, they are nonzero only for positive indices). However, we do leave the restriction that the filters are finitely supported, that is, they are FIR filters. ${ }^{2}$ Consider an inner product between two sequences $x$ and $y$ (on the left), and filtering a sequence $x$ by a filter $f$ and having the output at time $k$ (on the right):

$$
\langle y, x\rangle=\sum_{i \in \mathbb{Z}} y_{i} x_{i}^{*}, \quad(f * x)_{k}=\sum_{i \in \mathbb{Z}} f_{k-i} x_{i}^{*} .
$$

By comparing the above two expressions, we see that we could express filtering a sequence $x$ by a filter $f$ and having the output at time $k$ as:

$$
\sum_{i \in \mathbb{Z}} f_{k-i} x_{i}^{*}=\left\langle f_{k-i}, x_{i}\right\rangle
$$

To express the analysis part of the filter bank, we can do the following:

$$
X=\left(\begin{array}{c}
\vdots \\
X_{0} \\
X_{1} \\
X_{2} \\
X_{3} \\
\vdots
\end{array}\right)=\left(\begin{array}{c}
\vdots \\
\left\langle x, \tilde{\varphi}_{0}\right\rangle \\
\left\langle x, \tilde{\varphi}_{1}\right\rangle \\
\left\langle x, \tilde{\varphi}_{2}\right\rangle \\
\left\langle x, \tilde{\varphi}_{3}\right\rangle \\
\vdots
\end{array}\right)=\left(\begin{array}{c}
\vdots \\
\left\langle\tilde{g}_{-i}, x_{i}\right\rangle \\
\left\langle\tilde{h}_{-i}, x_{i}\right\rangle \\
\left\langle\tilde{g}_{2-i}, x_{i}\right\rangle \\
\left\langle\tilde{h}_{2-i}, x_{i}\right\rangle \\
\vdots
\end{array}\right)
$$

\footnotetext{
${ }^{2}$ IIR filters also fit in this framework, we concentrate on FIR only for simplicity. Moreover, this restriction makes all the operators bounded and all the series converge.
} 


$$
=\underbrace{\left(\begin{array}{cccccc}
\ddots & \vdots & \vdots & \vdots & \vdots & \ddots \\
\cdots & \tilde{g}_{3} & \tilde{g}_{2} & \tilde{g}_{1} & \tilde{g}_{0} & \cdots \\
\cdots & \tilde{h}_{3} & \tilde{h}_{2} & \tilde{h}_{1} & \tilde{h}_{0} & \cdots \\
\cdots & \tilde{g}_{5} & \tilde{g}_{4} & \tilde{g}_{3} & \tilde{g}_{2} & \cdots \\
\cdots & \tilde{h}_{5} & \tilde{h}_{4} & \tilde{h}_{3} & \tilde{h}_{2} & \cdots \\
\ddots & \vdots & \vdots & \vdots & \vdots & \ddots
\end{array}\right)}_{\tilde{\Phi}^{*}} \underbrace{\left(\begin{array}{c}
\vdots \\
x_{0} \\
x_{1} \\
x_{2} \\
x_{3} \\
\vdots
\end{array}\right)}_{x}=\tilde{\Phi}^{*} x .
$$

Similarly, the reconstruction part can be expressed as

$$
\begin{aligned}
& x=\underbrace{\left(\begin{array}{cccccccc}
\ddots & \vdots & \vdots & \vdots & \vdots & \vdots & \vdots & \ddots \\
\cdots & g_{2} & h_{2} & g_{0} & h_{0} & 0 & 0 & \cdots \\
\cdots & g_{3} & h_{3} & g_{1} & h_{1} & 0 & 0 & \cdots \\
\cdots & g_{4} & h_{4} & g_{2} & h_{2} & g_{0} & h_{0} & \cdots \\
\cdots & g_{5} & h_{5} & g_{3} & h_{3} & g_{1} & h_{1} & \cdots \\
\ddots & \vdots & \vdots & \vdots & \vdots & \vdots & \vdots & \ddots
\end{array}\right)}_{\Phi} \underbrace{\left(\begin{array}{c}
\vdots \\
X_{0} \\
X_{1} \\
X_{2} \\
X_{3} \\
\vdots
\end{array}\right)}_{X}
\end{aligned}
$$

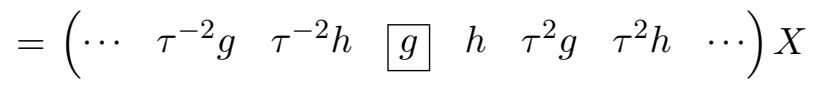

$$
\begin{aligned}
& =\left(\begin{array}{ccccc}
\ddots & \vdots & \vdots & \vdots & \ddots \\
\cdots & \Phi_{1} & \Phi_{0} & 0 & \cdots \\
\cdots & \Phi_{2} & \Phi_{1} & \Phi_{0} & \cdots \\
\ddots & \vdots & \vdots & \vdots & \ddots
\end{array}\right) X=\Phi X
\end{aligned}
$$

where $\Phi_{i}$ are $m \times m$ matrices, with $m$ denoting both the shift and the number of channels/filters in the filter bank. The matrices are formed by taking the $i$ th block of $m$ coefficients from each of the $m$ filters. Here $m=2$. From above, we can conclude the following:

(1) The basis is $\Phi=\left\{\tau^{2 i} \varphi_{0}, \tau^{2 i} \varphi_{1}\right\}_{i \in \mathbb{Z}}=\left\{\tau^{2 i} g, \tau^{2 i} h\right\}_{i \in \mathbb{Z}}$. In other words, the impulse responses of the template filters $g$ and $h$ and their even shifts form the basis $\Phi$ (they are the columns of $\Phi)$.

(2) The dual basis is $\tilde{\Phi}=\left\{\tau^{2 i} \tilde{\varphi}_{0}, \tau^{2 i} \tilde{\varphi}_{1}\right\}_{i \in \mathbb{Z}}=\left\{\tau^{2 i} \tilde{g}^{*}, \tau^{2 i} \tilde{h}^{*}\right\}_{i \in \mathbb{Z}}$. In other words, the impulse responses of the template filters 
$\tilde{g}^{*}$ and $\tilde{h}^{*}$ and their even shifts form the basis $\tilde{\Phi}$ (they are the columns of $\tilde{\Phi})$.

(3) When $\tilde{\Phi}=\Phi$, the basis is orthonormal. In that case, $\tilde{g}_{i}=g_{-i}$, that is, the impulse responses of the analysis filters are timereversed impulse responses of synthesis filters.

(4) The even shifts appear because of down/upsampling by 2 .

(5) When the filters are of length $l=2\left(l=m\right.$ in general), $\Phi^{*}$ or $\tilde{\Phi}^{*}$ contain only one block, $\Phi_{0}^{*}$ or $\tilde{\Phi}_{0}^{*}$, along the diagonal, making it a block-diagonal matrix (as in the Haar transform). The effect of this is that the input is processed in nonoverlapping pieces of length 2. Effectively, this is equivalent to dealing with bases in the two-dimensional space.

(6) We discussed here a specific case with 2 template filters and shifts by 2 . In filter bank parlance, we discussed two-channel filter banks with sampling ${ }^{3}$ by 2 . Of course, more general options are possible and one can have $m$-channel filter banks with sampling by $m$. We then have $m$ template filters (basis vectors) from which all the basis vectors are obtained by shifts by multiples of $m$. The blocks $\Phi_{i}^{*}$ then become of size $m \times m$. Again, if filters are of length $l=m$, this leads to the block-diagonal $\Phi^{*}$, and effectively, finite-dimensional bases.

\subsection{1 z-Domain View of Signal Processing}

Historically, the above, basis-centric view of filter banks came very recently. Initially, when the filter banks were developed to deal with speech coding $[57,83]$, the analysis was done in $z$-domain (for easier algebraic manipulation).

The mapping that takes us from the original domain to the $z$-domain is the $z$-transform, defined for a sequence $x \in \ell^{2}(\mathbb{Z})$ as

$$
X(z)=\sum_{i \in \mathbb{Z}} x_{i} z^{-i}
$$

You can think of the $z$-transform as a generalized discrete-time Fourier transform (DTFT), where $e^{j \omega}$ has been replaced by the complex

\footnotetext{
${ }^{3}$ By sampling, we mean the two sampling operations, downsampling and upsampling.
} 
number $z=r e^{j \omega}$. Just like the DTFT, the $z$-transform possesses nice properties (such as the convolution property) making it a useful analysis tool. More precisely, the $z$-transform allows us to deal with polynomiallike objects instead of convolutions.

In particular, $z$-transform comes in handy when we have to deal with shift-varying systems such as filter banks. Shift variance is introduced into the system due to downsamplers (or shifts). A tool used to transform a filter bank from a single-input single-output linear periodically shift-variant system into a multiple-input multiple-output linear shift-invariant systems is called the polyphase transform.

For $i=0, \ldots, m-1$, the $i$ th synthesis filter (template basis vector), $\left(\varphi_{i 0}(z), \ldots, \varphi_{i, m-1}(z)\right)^{T}$ is called the polyphase representation of the $i t h$ synthesis filter where

$$
\varphi_{i k}(z)=\sum_{p \in \mathbb{Z}} \varphi_{i, m p+k} z^{-p}
$$

are the polyphase components for $i, k=0, \ldots, m-1$. To relate $\varphi_{i k}(z)$ to a time-domain object, note that it is the discrete-time Fourier transform of the template basis vector $\varphi_{i}$ obtained by retaining only the indices congruent to $k$ modulo $m$. Then $\Phi_{p}(z)$ is the corresponding $m \times m$ synthesis polyphase matrix with elements $\varphi_{i k}(z)$. In other words, a polyphase decomposition is a decomposition into $m$ subsequences modulo $m$. We can do the same on the analysis side, leading to the polyphase matrix $\tilde{\Phi}_{p}^{*}(z)$. Then, the input/output relationship is given by

$$
x(z)=\left(1 z^{-1} \ldots z^{-(m-1)}\right) \Phi_{p}\left(z^{m}\right) \tilde{\Phi}_{p}^{*}\left(z^{m}\right) x_{p}(z),
$$

where $x_{p}(z)$ is the vector of polyphase components of the signal (there are $m$ of them) and ${ }^{*}$ denotes conjugation of coefficients but not of $z$. Note that the polyphase components of the analysis bank are defined in reverse order from those of the synthesis bank. When the filter length is $l=m$, then, each polyphase sequence is of length 1 . Each polyphase matrix then reduces to $\Phi_{p}(z)=\Phi_{0}, \tilde{\Phi}_{p}^{*}(z)=\tilde{\Phi}_{0}^{*}$, that is, both $\Phi_{p}(z)$ and $\tilde{\Phi}_{p}^{*}(z)$ become independent of $z$. It is clear from the above, that to obtain perfect reconstruction, that is, to have a basis expansion, the 
polyphase matrices must satisfy:

$$
\Phi_{p}(z) \tilde{\Phi}_{p}^{*}(z)=I .
$$

If the filter length is $l=m$, the above implements a finite-dimensional expansion (block transform). For example, if we want to implement the $\mathrm{DFT}_{m}$ using a filter bank, we would use an $m$-channel filter bank with sampling by $m$, and prototype synthesis filters $\varphi_{i}$ given in (2.9). Since each prototype filter is of length $m$, each of its polyphase components will be of length 1 and a constant, leading to a constant polyphase matrix.

If a filter bank implements an orthonormal basis, then $\tilde{\Phi}_{p}(z)=$ $\Phi_{p}\left(z^{-1}\right)$, and (5.6) reduces to

$$
\Phi_{p}(z) \Phi_{p}^{*}\left(z^{-1}\right)=I .
$$

A matrix satisfying the above is called a paraunitary matrix, that is, it is unitary on the unit circle.

Note that the polyphase transform is a discrete version of the wellknown Zak transform. Given that the Zak transform is unitary, it follows that the polyphase matrices are a matrix representation of the analysis/synthesis, providing yet another way to nicely transfer results between filter bank theory and frame theory.

Example 5.2. As a first example, go back to the Haar expansion discussed earlier. Since $m=2, \varphi_{0}(z)=\left(1+z^{-1}\right) / \sqrt{2}, \varphi_{1}(z)=$ $\left(1-z^{-1}\right) / \sqrt{2}$, and the polyphase matrix is $\Phi_{p}^{*}(z)=\Phi_{0}^{*}$ from (5.1).

As a more involved example, suppose $m=2$ again and we are given the following set of template filters:

$$
\begin{aligned}
G(z) & =z^{-2}+4 z^{-1}+6+4 z+z^{2} \\
H(z) & =\frac{1}{4} z\left(\frac{1}{4} z^{-1}+1+\frac{1}{4} z\right) \\
\tilde{G}(z) & =\frac{1}{4}\left(-\frac{1}{4} z^{-1}+1-\frac{1}{4} z\right) \\
\tilde{H}(z) & =z^{-1}\left(z^{-2}-4 z^{-1}+6-4 z+z^{2}\right) .
\end{aligned}
$$

Having the polyphase decomposition for each filter being written as: $G(z)=G_{0}\left(z^{2}\right)+z^{-1} G_{1}\left(z^{2}\right), H(z)=H_{0}\left(z^{2}\right)+z^{-1} H_{1}\left(z^{2}\right)$, 
$\tilde{G}(z)=\tilde{G}_{0}\left(z^{2}\right)+z \tilde{G}_{1}\left(z^{2}\right), \quad \tilde{H}(z)=\tilde{H}_{0}\left(z^{2}\right)+z \tilde{H}_{1}\left(z^{2}\right)$, the polyphase matrices are then:

$$
\begin{aligned}
& \Phi_{p}(z)=\left(\begin{array}{ll}
G_{0}(z) & H_{0}(z) \\
G_{1}(z) & H_{1}(z)
\end{array}\right)=\left(\begin{array}{cc}
z^{-1}+6+z & \frac{1}{16}(1+z) \\
4(1+z) & \frac{1}{4} z
\end{array}\right) \\
& \tilde{\Phi}_{p}(z)=\left(\begin{array}{cc}
\tilde{G}_{0}(z) & \tilde{H}_{0}(z) \\
\tilde{G}_{1}(z) & \tilde{H}_{1}(z)
\end{array}\right)=\left(\begin{array}{cc}
\frac{1}{4} & -4\left(1+z^{-1}\right) \\
-\frac{1}{16}\left(1+z^{-1}\right) & 1+6 z^{-1}+z^{-2}
\end{array}\right) .
\end{aligned}
$$

Thus, the filter bank with filters as defined above implements a biorthogonal expansion. The dual bases are:

$$
\begin{aligned}
& \Phi=\left\{\varphi_{2 i}, \varphi_{2 i+1}\right\}_{i \in \mathbb{Z}}=\left\{\tau^{2 i} g, \tau^{2 i} h\right\}_{i \in \mathbb{Z}} \\
& \tilde{\Phi}=\left\{\tilde{\varphi}_{2 i}, \tilde{\varphi}_{2 i+1}\right\}_{i \in \mathbb{Z}}=\left\{\tau^{2 i} \tilde{g}, \tau^{2 i} \tilde{h}\right\}_{i \in \mathbb{Z}}
\end{aligned}
$$

and they are interchangeable.

\subsubsection{Filter Bank Trees}

Many of the bases in $\ell^{2}(\mathbb{Z})$ (and frames later on), are built by using two- and $m$-channel filter banks as building blocks. For example, the dyadic (with scale factor 2) discrete wavelet transform (DWT) is built by iterating the two-channel filter bank on the lowpass channel (Figure 5.2 depicts the synthesis part). The DWT is a basis expansion and as such nonredundant (critically sampled). To describe the redundancy of various frame families later on, we introduce sampling grids in Figure 6.2, each depicting time positions of basis vectors at each

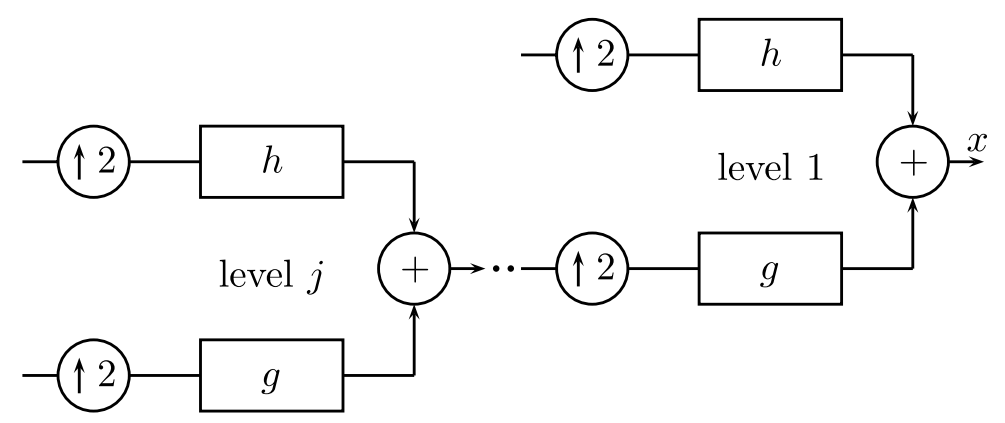

Fig. 5.2 The synthesis part of the filter bank implementing the DWT with $j$ levels. The analysis part is analogous. 
level. Thus, for example, the top plot in Figure 6.2 depicts the grid for the DWT. At level 1, we have half as many points as at level 0, at level 2, half as many as at level 1 , and so on. Because of appropriate sampling, the grid has exactly as many points as needed to represent any $x \in \ell^{2}(\mathbb{Z})$ and is thus nonredundant.

We can also build arbitrary trees by, at each level, iterating on any subset of the branches (typically known as wavelet packets [54]). In order to analyze these tree-structured filter banks, we typically collect all the filters and samplers along a path into a branch with a single filter and single sampler. This is possible using the so-called Noble identities [163] which allow us to exchange the order of filtering and sampling.

Example 5.3. Assume we have a DWT with 2 levels, that is, the lowpass branch is iterated only once as in Figure 5.2. Then, the equivalent filter bank has 3 channels as in Figure 5.3 with sampling by 2 , 4 , and 4 , respectively. The equivalent filters are then (call $(\uparrow m)$ the operator upsampling a filter by $m$ ):

$$
\varphi_{2}=h, \quad \varphi_{1}=g *(\uparrow 2) h, \quad \varphi_{0}=g *(\uparrow 2) g .
$$

Assuming for simplicity that the filters have only two taps, the matrix $\Phi$ in (5.2) is block diagonal with:

$$
\Phi_{0}^{*}=\left(\begin{array}{cccc}
h_{0} & h_{1} & 0 & 0 \\
0 & 0 & h_{0} & h_{1} \\
g_{0} h_{0} & g_{1} h_{0} & g_{0} h_{1} & g_{1} h_{1} \\
g_{0}^{2} & g_{0} g_{1} & g_{0} g_{1} & g_{1}^{2}
\end{array}\right)
$$

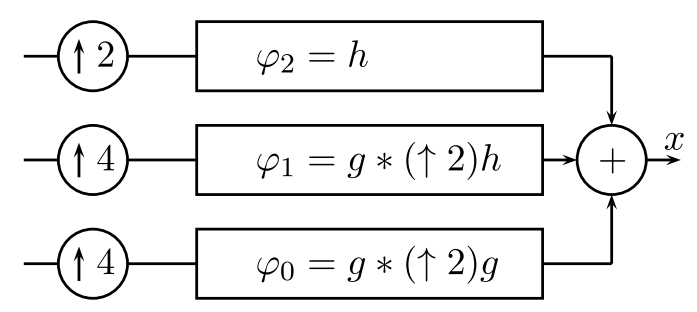

Fig. 5.3 The synthesis part of the equivalent three-channel filter bank implementing the DWT with 2 levels. The analysis part is analogous. 
We see here that even though we have only three branches, the filter bank behaves as a critically sampled four-channel filter bank with sampling by 4 .

\subsubsection{Summary: Bases $m=n$}

To summarize, the class of multiresolution transforms obtained using a filter bank depends on three parameters: the number of vectors $m$, the shift or sampling factor $n$ and the length $l$ of the nonzero support of the vectors:

When $m=n$, the filter bank is called critically sampled and implements a nonredundant expansion - basis. The basis $\Phi$ has a dual basis associated with it, $\tilde{\Phi}$, leading to biorthogonal filter banks. The associated matrices $\Phi, \tilde{\Phi}$ are invertible. In the $z$-domain, this is expressed as follows: A filter bank implements a basis expansion if and only if (5.6) evaluated on the unit circle is satisfied [166].

An important subcase is when the basis $\Phi$ is orthonormal, in which case it is self-dual, that is, $\tilde{\Phi}=\Phi$. The filter bank is called orthogonal and the associated matrix $\Phi$ is unitary, $\Phi \Phi^{*}=I$. In the $z$-domain, this is expressed as follows: A filter bank implements an orthonormal basis expansion if and only if its polyphase matrix is paraunitary, that is, if and only if (5.7) holds [166]. Well-known subcases are the following:

(1) When $l=m$, we have a block transform. In this case, in (5.2), only $\Phi_{0}$ exists, making $\Phi$ block-diagonal. In effect, since there is no overlap between processed signal blocks, this can be analyzed as a finite-dimensional case, where both the input and the output are $m$-dimensional vectors. A famous example is the DFT we discussed earlier.

(2) When $m=2$, we get two-channel filter banks. In (5.2), $\Phi_{i}$ is of size $2 \times 2$ and by iterating on the lowpass channel, we get the DWT [166] (see Figure 5.2).

(3) When $l=2 m$, we get lapped orthogonal transforms (LOT), efficient transforms developed to deal with the blocking artifacts introduced by block transforms, while keeping the efficient computational algorithm of the DFT [166]. In this case, in (5.2), only $\Phi_{0}$ and $\Phi_{1}$ are nonzero. 


\subsection{Frames via Filter Banks}

The filter bank expansions we just discussed were bases and thus nonredundant. Now, nothing stops us from being redundant (for reasons stated earlier) by simply adding more vectors.

Example 5.4 (Mercedes-Benz frame cont'd). Let us look at the simplest case using our favorite example: the Mercedes-Benz frame. Our $\Phi^{*}$ is now block-diagonal, with $\Phi_{0}^{*}=\Phi_{\mathrm{UNTF}}^{*}$ from (3.2) on the diagonal. In contrast to finite-dimensional bases implemented by filter banks as in (5.1), the block $\Phi_{0}^{*}$ is now rectangular of size $3 \times 2$. This finitedimensional frame is equivalent to the filter bank shown in Figure 5.4, with $\left\{\tilde{\varphi}_{i}\right\}=\left\{\varphi_{i}\right\}$, given in $(3.2)$.

As we could for finite-dimensional bases, we can investigate finitedimensional frames within the filter bank framework (see Figure 5.5). In other words, all cases we consider in this survey, both finite dimensional and infinite dimensional, we can look at as filter banks.

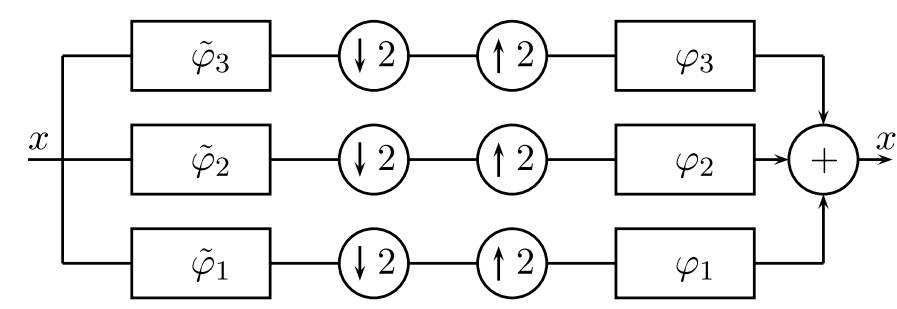

Fig. 5.4 Three-channel filter bank with downsampling by 2 .

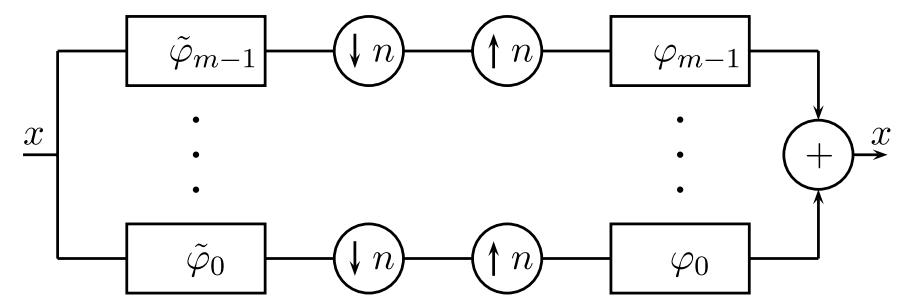

Fig. 5.5 A filter bank implementation of a frame expansion: It is an $m$-channel filter bank with sampling by $n$. 
Similarly to bases, if in (5.2) $\Phi$ is not block diagonal, we resort to the polyphase-domain analysis. Assume that the filter length is $l=k n$ (if not, we can always pad with zeros), and write the frame as (causal filters)

$$
\Phi^{*}=\left(\begin{array}{ccccccc}
\ddots & \vdots & \vdots & \vdots & \vdots & \vdots & \ddots \\
\cdots & \Phi_{0}^{*} & \Phi_{1}^{*} & \cdots & \Phi_{k-1}^{*} & 0 & \cdots \\
\cdots & 0 & \Phi_{0}^{*} & \cdots & \Phi_{k-2}^{*} & \Phi_{k-1}^{*} & \cdots \\
\cdots & \vdots & \vdots & \vdots & \vdots & \vdots & \cdots \\
\cdots & 0 & 0 & \cdots & \Phi_{0}^{*} & \Phi_{1}^{*} & \cdots \\
\cdots & 0 & 0 & \cdots & 0 & \Phi_{0}^{*} & \cdots \\
\ddots & \vdots & \vdots & \vdots & \vdots & \vdots & \ddots
\end{array}\right)
$$

where each block $\Phi_{i}$ is of size $n \times m$. $\Phi_{0}$, for example, is

$$
\Phi_{0}=\left(\begin{array}{ccc}
\varphi_{00} & \ldots & \varphi_{0, m-1} \\
\vdots & \ddots & \vdots \\
\varphi_{n-1,0} & \ldots & \varphi_{n-1, m-1}
\end{array}\right) .
$$

In the above, we enumerate template frame vectors from $0, \ldots, m-1$. A thorough analysis of oversampled filter banks seen as frames is given in $[29,61,62]$.

An interesting twist is to consider transmultiplexers [11], obtained by starting by a synthesis filter bank and following it with an analysis one [166]. This scheme is of great importance in practice as it is a basis for frequency-division multiplexing, OFDM, for example. While transmultiplexers are almost exclusively studied for filter-bank bases, nothing prevents us from doing the same with frames. Similar ideas can be found in the continuous-time setting in $[68,111]{ }^{4}$

\subsubsection{Summary: Frames $m>n$}

When $m>n$, the filter bank implements a redundant expansion frame. The frame $\Phi$ has a dual frame associated with it, $\tilde{\Phi}$. The associated matrices $\Phi, \tilde{\Phi}$ are rectangular and left/right invertible. This has

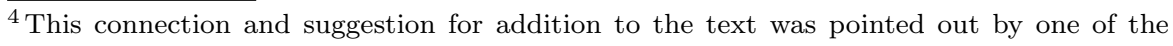
reviewers of this survey.
} 
been formalized in $z$-domain in [61], as the following result: A filter bank implements a frame decomposition in $\ell^{2}(\mathbb{Z})$ if and only if its polyphase matrix is of full rank on the unit circle.

An important subcase is when $\Phi$ is tight, in which case it can be self-dual, and thus, $\tilde{\Phi}=\Phi$, and $\Phi \Phi^{*}=I$. This has been formalized in $z$-domain in [61], as the following result: A filter bank implements a tight frame expansion in $\ell^{2}(\mathbb{Z})$ if and only if its polyphase matrix is paraunitary. A well-known subcase of tight frames is the following: When $l=n$, we have a block transform. Then, in (5.8), only $\Phi_{0}$ is nonzero, making $\Phi$ block-diagonal. In effect, since there is no overlap between processed blocks, this can be analyzed as a finite-dimensional case, where both the input and the output are $n$-dimensional vectors. 
We now consider particular frame families. The first two, harmonic tight frames and equiangular frames are purely finite dimensional, while the rest are, in general, infinite dimensional. For some of the families, we will consider the unit-norm tight frame version and give the frame bound $A$ yielding the redundancy of the frame family. ${ }^{1}$

\subsection{Harmonic Tight Frames and Variations}

Harmonic tight frames are obtained by seeding from $\Psi=\mathrm{DFT}_{m}^{*}$ given in $(2.8)-(2.10)$, by deleting the last $(m-n)$ columns:

$$
\varphi_{i}=\sqrt{\frac{m}{n}}\left(W_{m}^{0}, W_{m}^{i}, \ldots, W_{m}^{i(n-1)}\right)
$$

for $i=0, \ldots, m-1$. Since obtained as an instance of the Naimark theorem, this is thus a Parseval tight frame, that is, $\Phi \Phi^{*}=I$. The simplest

\footnotetext{
$\overline{{ }^{1} \text { We will denote by }} A_{j}$ the redundancy/frame bound at level $j$ when iterated filter banks are used.
} 
example of a harmonic tight frame is the Mercedes-Benz frame we introduced in Section 3.

In [41], the authors define a more general version of the harmonic tight frame, called general harmonic frames as follows:

$$
\varphi_{k}=\left(c_{1}^{k} b_{1}, c_{2}^{k} b_{2}, \ldots, c_{n}^{k} b_{n}\right),
$$

for $k=0, \ldots, m-1$, with $|c|=1,\left|b_{i}\right|=\frac{1}{\sqrt{m}}(1 \leq i \leq n)$, and $\left\{c_{i}\right\}_{i=1}^{n}$ being distinct $m$ th roots of $c$. They also show that the harmonic tight frames are unique up to a permutation of the orthonormal basis and that every general harmonic frame is unitarily equivalent to a simple variation of a harmonic tight frame.

Harmonic tight frames have a number of interesting properties: (a) For $m=n+1$, all equal-norm tight frames are unitarily equivalent to it; in other words, since we have harmonic tight frames for all $n, m$, we have all equal-norm tight frames for $m=n+1$. (b) It is the only equal-norm Parseval tight frame such that its elements are generated by a group of unitary operators with one generator. (c) Harmonic tight frames are maximally robust to erasures [92].

These frames have been generalized in an exhaustive work by Vale and Waldron [164], where the authors look at frames with symmetries. Some of these they term harmonic tight frames (their definition is more general than what is given in (6.1)), and are the result of the operation of a unitary $U$ on a finite Abelian group $G$. When $G$ is cyclic, the resulting frames are cyclic. In [41], the harmonic tight frames we showed above are with $U=I$ and generalized harmonic tight frames are with $U=D$ diagonal. These are cyclic in the parlance of [164]. An example of a cyclic frame are $(n+1)$ vertices of a regular simplex in $\mathbb{R}^{n}$. There exist harmonic tight frames which are not cyclic.

Similar ideas have appeared in the work by Eldar and Bölcskei [79] under the name geometrically uniform frames, frames defined over a finite Abelian group of unitary matrices both with a single generator as well as multiple generators. The authors also consider constructions of such frames from given frames, closest in the least-squares sense, a sort of a "Gram-Schmidt" procedure for geometrically uniform frames. 


\subsection{Grassmanian Packings and Equiangular Frames}

\subsubsection{Equiangular Frames}

Equiangular frames are those frames with $\left|\left\langle\varphi_{i}, \varphi_{j}\right\rangle\right|$ is a constant. They have become popular recently due to their use in quantum computing. ${ }^{2}$ In that terminology, a rank-1 measurement is represented by a positive operator valued measure (POVM). Each rank-1 POVM is a tight frame. ${ }^{3}$

The first family is symmetric informationally complete POVMs (SIC-POVMs) [141]. An SIC-POVM is a family $\Phi$ of $m=n^{2}$ vectors in $\mathbb{C}^{n}$ such that

$$
\left|\left\langle\varphi_{i}, \varphi_{j}\right\rangle\right|^{2}=\frac{1}{n+1}
$$

holds for all $i, j, i \neq j$. At this point, it is not known whether SICPOVMs exist for all finite dimensions.

The second family are mutually unbiased bases, with applications in various problems, cryptography among them [82]. Mutually unbiased bases form a family $\Phi$ of $(n+1)$ orthonormal bases in a Hilbert space of dimension $n$ (for instance, $\mathbb{C}^{n}$ ) such that for any two different bases $B_{I}, B_{J}$ and any vectors $\varphi_{i} \in B_{I}$ and $\varphi_{j} \in B_{J}$, we have

$$
\left|\left\langle\varphi_{i}, \varphi_{j}\right\rangle\right|^{2}=\frac{1}{n}
$$

Equiangular tight frames have proven very useful in communications, coding theory, and sparse approximation [157, 160]. In [105], Holmes and Paulsen show that these frames give error correction codes that are maximally robust against two erasures, whereas Bodmann and Paulsen [25] examine the general case of an arbitrary number of erasures (see also Section 7.4 for a brief overview of robust transmission).

\footnotetext{
2 "A single qubit measurement corresponds to a tight frame in the two-dimensional Hilbert space $\mathbb{H}$, while an $n$-qubit measurement corresponds to a tight frame in the $2^{n}$ dimensional $n$-fold Kronecker product of $\mathbb{H}$. An $n$-qubit measurement itself does not have to be an $n$ fold Kronecker product of $n$ single qubit measurements; however when that is the case, the $n$-qubit measurement is obtained by $n$ independent measurements on single qubits. This would have to be done when dealing with physically separated (even possibly entangled) qubits. Therefore, Kronecker tight frames describe independent single qubit measurements in multiple qubit systems" [154, 153].

${ }^{3}$ Note that POVMs do not have to be equiangular.
} 
In [158], the authors provide theoretical results that show for which pairs $(m, n)$ do equiangular real tight unit-norm frames exist. They also study the complex case. Recently, Bodmann et al. [24] have used mutually unbiased bases for linear reconstruction of signals when all phase information is lost and only the magnitudes of the frame coefficients remain.

\subsubsection{Grassmanian Packings}

Both harmonic tight frames and equiangular frames have strong connections to Grassmanian frames. In a comprehensive paper [157], Strohmer and Heath discuss those frames and their connection to Grassmanian packings, spherical codes, graph theory, and Welch bound sequences (see also [105]). These frames are of unit norm (not a necessary restriction) and minimize the maximum correlation $\left|\left\langle\varphi_{i}, \varphi_{j}\right\rangle\right|$ among all frames. The problem arises from looking at overcomplete systems closest to orthonormal bases (which have minimum correlation). A simple example is a harmonic tight frame in $\mathbb{H}^{n}$. Theorem 2.3 in [157] states that, given a frame $\Phi$ :

$$
\min _{\Phi}\left(\max _{\left(\varphi_{i}, \varphi_{j}\right)}\left|\left\langle\varphi_{i}, \varphi_{j}\right\rangle\right|\right) \geq \sqrt{\frac{m-n}{n(m-1)}} .
$$

The equality in (6.4) is achieved if and only if $\Phi$ is equiangular and tight. In particular, for $\mathbb{H}=\mathbb{R}$, equality is possible only for $m \leq n(n+1) / 2$, while for $\mathbb{H}=\mathbb{C}$, equality is possible only for $m \leq n^{2}$. Note that the above inequality is exactly the one Welch proved in [171] and which later lead to what is today commonly referred to as the Welch bound given in (7.4) by minimizing interuser interference in a CDMA system [131] (see the discussion on the Welch bound in Section 7.6). In a more recent work, Xia et al. [175] constructed some new frames meeting the original Welch bound (7.4).

These frames coincide with some optimal packings in Grassmanian spaces [55], spherical codes [56], equiangular lines [126], and many others. The equiangular lines are equivalent to the SIC-POVMs we discussed above. 


\section{All in the Family}

\subsection{The Algorithme à Trous}

The algorithme à trous ${ }^{4}$ is a fast implementation of the dyadic continuous wavelet transform. It was first introduced by Holschneider et al. in 1989 [106]. The transform is implemented via a biorthogonal, nondownsampled filter bank. An example for $j=2$ levels is given in Figure 6.1 (this is essentially the same as the 2-level DWT from Figure 5.3 with samplers removed).

Let $g$ and $h$ be the filters used in this filter bank. At level $i$ we will have equivalent upsampling by $2^{i}$ which means that the filter moved across the upsampler will be upsampled by $2^{i}$, inserting $\left(2^{i}-1\right)$ zeros between every two samples and thus creating holes.

The bottom plot in Figure 6.2 shows the sampling grid for the à trous algorithm. It is clear from the figure, that this scheme is completely redundant, as all the points exist. This is in contrast to a completely nonredundant scheme such as the DWT, given in the top plot of the same figure. In fact, while the redundancy per level of this algorithm grows exponentially since $A_{1}=$ $2, A_{2}=4, \ldots, A_{j}=2^{j}, \ldots$, the total redundancy for $j$ levels is linear, as $A=A_{j} 2^{-j}+\sum_{i=1}^{j} A_{i} 2^{-i}=(j+1)$. This growing redundancy is the price we pay for shift invariance as well as the simplicity of the algorithm. The $2 \mathrm{D}$ version of the algorithm is obtained by extending the $1 \mathrm{D}$ version in a separable manner, leading to the total redundancy of $A=A_{j} 2^{-j}+3 \sum_{i=1}^{j} A_{i} 2^{-i}=(3 j+1)$.

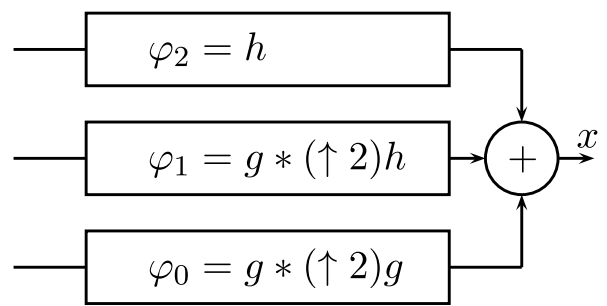

Fig. 6.1 The synthesis part of the filter bank implementing the à trous algorithm. The analysis part is analogous. This is equivalent to Figure 5.3 with sampling removed.

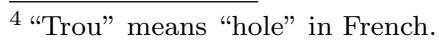



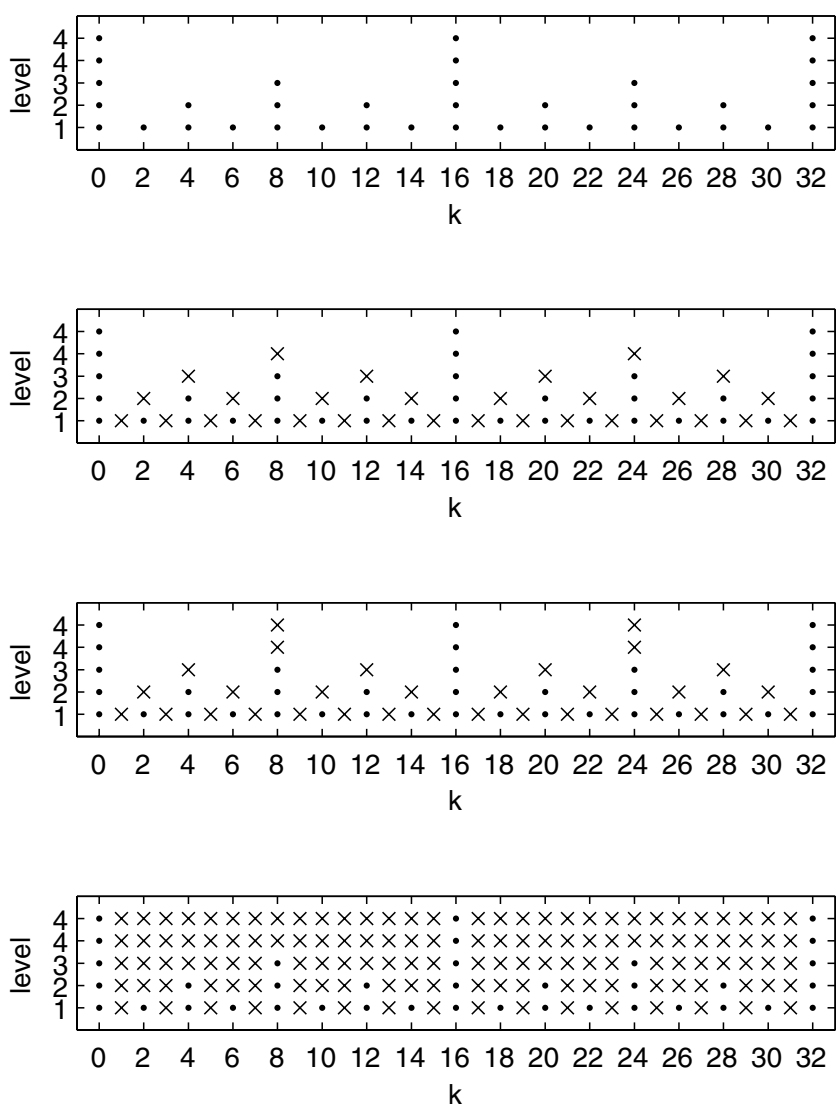

Fig. 6.2 Sampling grids corresponding to time-frequency tilings of (top to bottom): DWT (nonredundant), double-density DWT/Laplacian pyramid, double-tree CWT/powershiftable DWT/partial DWT, à trous family (completely redundant). Black dots correspond to the nonredundant, DWT-like sampling grid. Crosses denote redundant points. The last two ticks on the $y$-axis are both for level 4, one for the highpass and the other for the lowpass channel, respectively.

\subsection{Gabor and Cosine-Modulated Frames}

The idea behind this class of frames, consisting of many families, dates back to Gabor [90] and the insight of constructing bases by modulation of a single prototype function. Gabor originally used complex modulation, and thus, all those families with complex modulation are termed Gabor frames. Other types of modulation are possible, such as cosine modulation, and again, all those families with cosine modulation 
are termed cosine-modulated frames. ${ }^{5}$ The connection between these two classes is deep as there exists a general decomposition of the frame operator corresponding to a cosine-modulated filter bank as the sum of the frame operator of the underlying Gabor frame (with the same prototype function and twice the redundancy) and an additional operator, which vanishes if the generator satisfies certain symmetry properties. While this decomposition has first been used by Auscher in the context of Wilson bases [5], it is valid more generally. Both of these classes can be seen as general oversampled filter banks with $m$ channels and sampling by $n$ (see Figure 5.5).

\subsubsection{Gabor Frames}

A Gabor frame is $\Phi=\left\{\varphi_{i}\right\}_{i=0}^{m-1}$, with

$$
\varphi_{i, k}=W_{m}^{-i k} \varphi_{0, k}
$$

where index $i=0, \ldots, m-1$ refers to the number of frame elements, $k \in \mathbb{Z}$ is the discrete-time index, $W_{m}$ is the $m$ th root of unity and $\varphi_{0}$ is the prototype frame function. Comparing (6.5) with (6.1), we see that for filter length $l=n$ and $\varphi_{0, k}=1, k=0$ and 0 otherwise, the Gabor system is equivalent to a harmonic tight frame. Thus, it is sometimes called the oversampled DFT frame.

For the critically sampled case it is known that one cannot have Gabor bases with good time and frequency localization at the same time (this is similar in spirit to the Balian-Low theorem which holds for $\mathcal{L}^{2}(\mathbb{R})[64]$ ); this prompted the development of oversampled (redundant) Gabor systems (frames). They are known under various names: oversampled DFT filter banks, complex-modulated filter banks, shorttime Fourier filter banks and Gabor filter banks, and have been studied in $[26,27,29,59,86]$ (see also [156] and references within). More recent work includes [125], where the authors study finite-dimensional Gabor systems and show a family in $\mathbb{C}^{n}$, with $m=n^{2}$ vectors, which allows for $n^{2}-n$ erasures, where $n$ is prime (see Section 7.4 for discussion of erasures). In [120], new classes of Gabor equal-norm tight frames are shown, which are also maximally robust.

\footnotetext{
${ }^{5}$ Cosine-modulated bases are also often called Wilson bases.
} 


\subsubsection{Cosine-Modulated Frames}

The other kind of modulation, cosine, was used with great success within critically sampled filter banks due to efficient implementation algorithms. Its oversampled version was introduced in [27], where the authors define the frame elements as:

$$
\varphi_{i, k}=\sqrt{2} \cos \left(\frac{(i+1 / 2) \pi}{m}+\alpha_{i}\right) \varphi_{0, k}
$$

where index $i=0, \ldots, m-1$ refers to the number of frame elements, $k \in \mathbb{Z}$ is the discrete-time index and $\varphi_{0}$ is the template frame function. Equation (6.6) defines the so-called odd-stacked cosine modulated filter banks; even-stacked ones exist as well.

Cosine-modulated filter banks do not suffer from time-frequency localization problems, given by a general result stating that the generating window of an orthogonal cosine modulated filter bank can be obtained by constructing a tight complex filter bank with oversampling factor 2 while making sure the window function satisfies a certain symmetry property (for more details, see [27]). Since we can get well-localized Gabor frames for redundancy 2, this also shows that we can get well-localized cosine-modulated filter banks. Malvar [130] constructed the modulated complex lapped transform of redundancy 2 based on the lapped orthogonal transform with basis functions similar to Princen-Johnson-Bradley filters [138].

\subsection{The Dual-Tree $\mathbb{C W T}$ and the Dual-Density DWT}

\subsubsection{The Dual-Tree $\mathbb{C W T}$}

The dual-tree complex wavelet transform (dual-tree $\mathbb{C} W T$ ) was first introduced by Kingsbury in 1998 [113, 114, 115]. The basic idea is to have two DWT trees working in parallel. One tree represents the real part of the complex transform while the second tree represents the imaginary part. That is, when the dual-tree $\mathbb{C W T}$ is applied to a real signal, the output of the first tree is the real part of the complex transform whereas the output of the second tree is its imaginary part. Shown in Figure 6.3 is the analysis and synthesis filter bank for the 


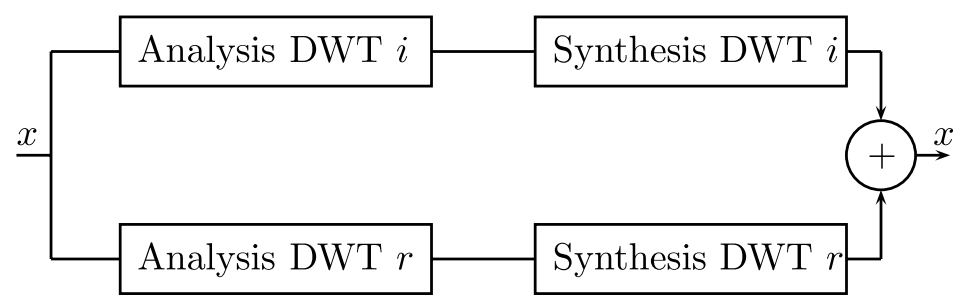

Fig. 6.3 The filter bank implementing the $\mathbb{C W T}$. The two branches have two different twochannel filter banks as in Figure 5.1. The analysis part is analogous.

dual-tree $\mathbb{C W T}$. Each tree uses a different pair of lowpass and highpass filters. These filters are designed so that they satisfy the perfect reconstruction condition (5.6).

Let $\Phi_{r}$ and $\Phi_{i}$ be the square matrices representing each of the DWTs in the dual-tree $\mathbb{C W T}$. Then,

$$
\Phi=\frac{1}{\sqrt{2}}\left(\Phi_{r} \Phi_{i}\right)
$$

is a rectangular matrix, and thus a frame, representing the dual-tree CWT. ${ }^{6}$ The right inverse of $\Phi$ is the analysis filter bank (analysis operator) and is given by $\tilde{\Phi}^{*}=1 / \sqrt{2}\left[\left(\Phi_{r}\right)^{-1}\left(\Phi_{i}\right)^{-1}\right]^{T}$. If $\Phi_{r}$ and $\Phi_{i}$ are unitary matrices, then $\tilde{\Phi}=\Phi, \Phi \Phi^{*}=I$, and $\Phi$ is a Parseval tight frame.

Because the two DWT trees used in the dual-tree $\mathbb{C W T}$ are fully downsampled, the redundancy is only 2 for the $1 \mathrm{D}$ case (it is $2^{d}$ for the $d$-dimensional case). We can see that in the third plot in Figure 6.2, where the redundancy at each level is twice that of the DWT, that is $A_{1}=A_{2}=\cdots=A_{j}=2$. Unlike the à trous algorithm, however, here the redundancy is independent of the number of levels used in the transform.

When the two DWTs used are orthonormal, the dual-tree $\mathbb{C W T}$ is a tight frame. The dual-tree $\mathbb{C W T}$ overcomes one of the main drawbacks of the DWT: shift variance. Since the dual-tree $\mathbb{C W T}$ contains two fully downsampled DWTs which satisfy the half-sample delay condition (see below), aliasing due to downsampling can be largely eliminated and the transform becomes nearly shift invariant. An advantage that the

$\overline{{ }^{6} \text { The indices } r \text { and }} i$ stem from real and imaginary. 
dual-tree $\mathbb{C W T}$ has over other complex transforms is that it has a fast invertible implementation and moreover, when the signal is real valued, the real and imaginary parts of its transform coefficients can be computed and stored separately.

As mentioned previously, the pairs of filters $\left(h_{r}, g_{r}\right)$ and $\left(h_{i}, g_{i}\right)$ of each DWT have to satisfy the perfect reconstruction condition. In addition, the filters have to be FIR and satisfy the so-called half sample delay condition, which implies that all of the filters have to be designed simultaneously. From this condition it also follows that the two highpass filters form an approximate Hilbert transform pair, and it thus makes sense to regard the outputs of the two trees as the real and imaginary parts of complex functions [145]. Different design solutions exist, amongst them the linear phase biorthogonal one and the quarter-shift one $[115,147]$. Moreover, we can use different-flavor trees to implement the dual-tree $\mathbb{C W T}$. For example, it is possible to use a different pair of filters at each level, or alternate filters between the trees at each stage except for the first one.

In two or more dimensions, the dual-tree $\mathbb{C W T}$ possesses directional selectivity allowing us to capture edge or curve information, a property clearly absent from the usual separable DWT. In the real case, orientation selectivity is simply achieved by using two real separable 2D DWTs in parallel. Two pairs of filters are used to implement each DWT. These two transforms produce six subbands, three pairs of subbands from the same space-frequency region. By taking the sums and differences of each pair, one obtains the oriented wavelet transform.

The near shift invariance and orientation selectivity properties of the dual-tree $\mathbb{C W T}$ open up a window into a wide range of applications, among them denoising, motion estimation, image segmentation as well as building feature, texture and object detectors for images (see Section 7.8 and references therein).

\subsubsection{The Double-Density DWT and Variations}

The dual-tree $\mathbb{C W T}$ appears to be the most popular among the oversampled filter bank transforms. It is joined by a host of others: In particular, Selesnick in [144] introduces the double-density DWT, which 
can approximately be implemented using a three-channel filter bank with sampling by 2 as in Figure 5.4. The filters in the analysis bank are time-reversed versions of those in the synthesis bank. The redundancy of this filter bank tends toward 2 when iterated on the channel with $\varphi_{1}$. Actually, we have that $A_{1}=\frac{3}{2}, A_{2}=\frac{7}{4}, \ldots, A_{\infty}=2$ (see second plot in Figure 6.2). Like the dual-tree $\mathbb{C W T}$, the double-density DWT is nearly shift invariant when compared to the à trous construction. In [146], Selesnick introduces the combination of the double-density DWT and the dual-tree $\mathbb{C W T}$ which he calls double-density, dual-tree $\mathbb{C} W T$. This transform can be seen as the one in Figure 6.3 (dualtree $\mathbb{C W T}$ ), with individual filter banks being overcomplete ones given in Figure 5.4 (double-density DWT). In [1], Abdelnour and Selesnick introduce symmetric, nearly shift-invariant filter banks implementing tight frames. These filter banks have four filters in two couples, obtained from each other by modulation. Sampling is by 2 and thus the total redundancy is 2 .

Another variation on a theme is the power-shiftable DWT [150] or partial DWT [155], which removes samplers at the first level but leaves them at all other levels (see Figure 6.4). The sampling grid of the power-shiftable DWT/partial DWT is shown in the third plot in Figure 6.2. We see that is has redundancy $A_{j}=2$ at each level, similarly to the $\mathbb{C W T}$. The power-shiftable DWT/partial DWT achieves near shift invariance.

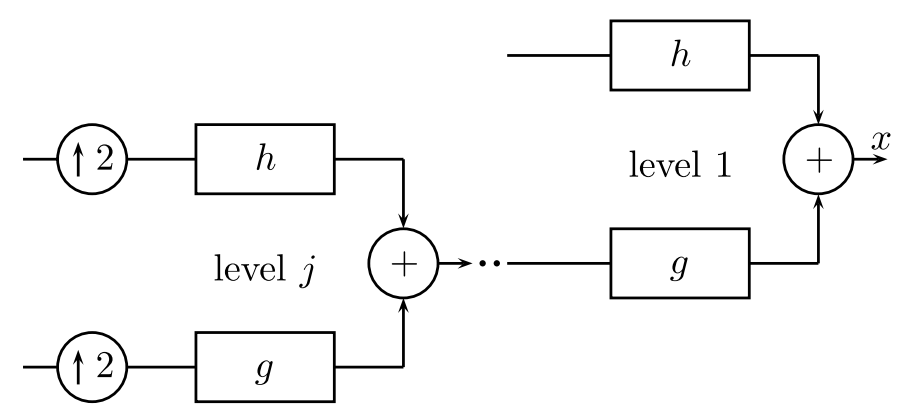

Fig. 6.4 The synthesis part of the filter bank implementing the power-shiftable DWT. The samplers are omitted at the first level but exist at all other levels. The analysis part is analogous. 
Bradley in [32] introduces overcomplete DWT, the DWT with critical sampling for the first $k$ levels followed by à trous for the last $j-k$ levels. The overcomplete DWT becomes the à trous algorithm when $k=0$ or the DWT when $k=j$. Bayram and Selesnick [10] develop an overcomplete DWT with rational scaling factors that is a tight frame. This construction allows for the resolution to increase more gradually (as opposed to usual dyadic schemes where the resolution is doubled) from each scale to the next finer scale. It also presents the advantage of being a nearly shift-invariant transform with FIR filters that can be easily designed to have a specified number of vanishing moments.

\subsection{Multidimensional Frames}

Apart from obvious, tensor-like, constructions (separate application of 1D methods in each dimension) of multidimensional frames, we are interested in true multidimensional solutions. The oldest multidimensional frame seems to be found in pyramid coding introduced by Burt and Adelson [33], which we discuss next.

\subsubsection{Pyramid Coding}

Pyramid coding was introduced in 1983 by Burt and Adelson [33]. Although redundant, the pyramid coding scheme was developed for compression of images and was recognized in the late 1980s as one of the precursors of wavelet octave-band decompositions. The scheme works as follows: First, a coarse approximation is derived (an example

of how this could be done is in Figure 6.5). While in Figure 6.5 the

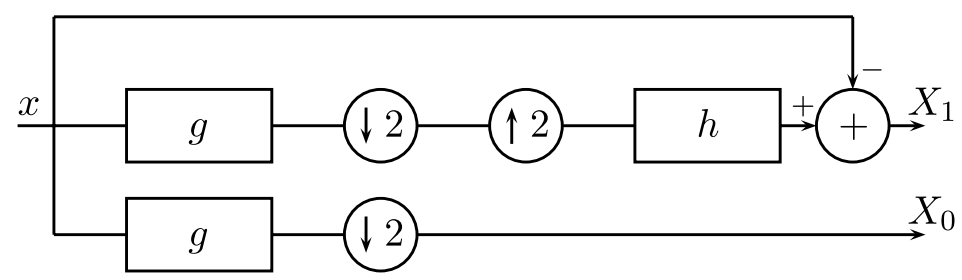

Fig. 6.5 The analysis part of the pyramid filter bank [33] with orthonormal filters $g$ and $h$, corresponding to a tight frame. 
intensity of the coarse approximation $X_{0}$ is obtained by linear filtering and downsampling, this need not be so; in fact, one of the powerful features of the original scheme is that any operator can be used, not necessarily linear. Then, from this coarse version, the original is predicted (in the figure, this is done by upsampling and filtering) followed by calculating the prediction error $X_{1}$. Since the prediction $X_{1}$ has smaller power than $x$ and has unit gain in the reconstruction process, it can be compressed more easily than $x$. The process can be iterated on the coarse version. In the absence of quantization of $X_{1}$, the original is obtained by simply adding back the prediction at the synthesis side.

The pyramid coding scheme is fairly intuitive and effective, thus its success. There are several advantages to pyramid coding: The quantization error depends only on the last quantizer in the iterated scheme. As we mentioned above, nonlinear operators can be used, opening the door to the whole host of possibilities (edge detectors, ....). The redundancy in $2 \mathrm{D}$ is only 1.33 , far less then the à trous construction, for example. Thanks to the above, pyramid coding has been recently used together with directional coding to form the basis for nonseparable multidimensional frames called contourlets (see Section 6.6.3).

\subsubsection{Steerable Pyramid}

The steerable pyramid was introduced by Simoncelli et al. in 1992 [150], following on the pyramid coding work. The steerable pyramid possesses many nice properties, such as joint space-frequency localization, approximate shift invariance, approximate tightness, oriented kernels, approximate rotation invariance and a redundancy factor of $4 j / 3$, where $j$ is the number of orientation subbands. The transform is implemented by a first stage of lowpass/highpass filtering followed by oriented bandpass filters in the lowpass branch plus another lowpass filter in the same branch followed by downsampling. In [136], Portilla and Simoncelli use a complex version of the steerable pyramid for texture modeling. An excellent overview of the steerable pyramid and its applications is given on Simoncelli's web page [149]. 


\subsubsection{Contourlets}

Another beautiful example is the recent work of Do and Vetterli on contourlets $[58,72]$. This work was motivated by the need to construct efficient and sparse representations of intrinsic geometric structure of information within an image. The authors combine the ideas of pyramid coding (see Section 6.6.1) and pyramid filter banks [71] with directional processing, to obtain contourlets - expansions capturing contour segments. The transform is a frame composed of a pyramid filter bank and a directional filter bank. Thus, first a wavelet-like method is used for edge detection (pyramid) followed by local directional transform for contour segment detection. It is almost critically sampled, with redundancy of 1.33. It draws on the ideas of a pyramidal directional filter bank which is a Parseval tight frame when all the filters used are orthogonal (see Figure 6.6).

\subsubsection{More Multidimensional Families}

Some other examples include [127] where the authors build both critically sampled and nonsampled (à trous like) 2D DWT. It is obtained by a separable 2D DWT producing four subbands. The lowest subband is left as is, while the three higher ones are split into two subbands each using a quincunx filter bank (checkerboard sampling). The resulting filter bank possesses good directionality with low redundancy. Many "-lets" are also multidimensional frames, such as curvelets $[34,35]$ and

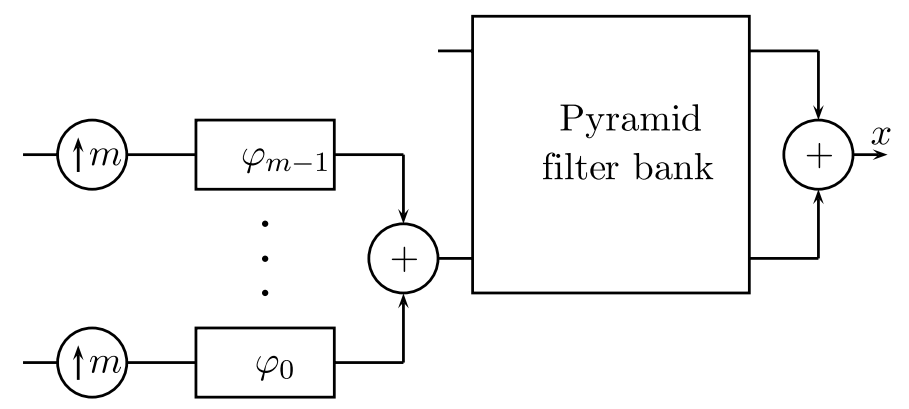

Fig. 6.6 The synthesis part of the pyramid directional filter bank. The pyramid filter bank is given in Section 6.6.1. The scheme can be iterated and the analysis part is analogous. 
shearlets [122]. As the name implies, curvelets are used to approximate curved singularities in an efficient manner [34, 35]. As opposed to wavelets which use dilation and translation, shearlets use dilation, shear transformation and translation, and possess useful properties such as directionality, elongated shapes, and many others [122].

\subsection{Discussion and Notes}

We finish this section with more recent developments; while some of them are not necessarily yet in the realm of filter-bank frames, they relate to them nevertheless.

For example, Casazza and Leonhard keep a tab on all equal-norm Parseval frames in [46]. In $[8,9,95]$, the authors introduce the notion of localized frames, as an important new direction in frame theory, with possible filter bank instantiations in the future. In [42, 44], Casazza and Kutyniok introduced fusion frames to model systems requiring distributed processing such as sensor networks. A fusion frame is a frame-like collection of low dimensional subspaces where each signal is represented by a collection of vector coefficients that are the projection of the signal onto the orthogonal bases of the fusion frame subspaces.

Fusion frames already found applications in distributed sensing [142], packet encoding [23], and the robustness of fusion frames against erasures has been studied in [43, 23]. The authors in [121] study optimally robust fusion frames against erasures and noise for random signals and show that in fact these optimal fusion frames, a class termed equidistance tight fusion frames, are optimal Grassmannian packings. For complete and up-to-date information concerning fusion frames, consult [45]. The authors of this survey have recently introduced lapped tight frame transforms, obtained by seeding the orthogonal lapped transforms [49]. 


\section{Applications}

We now look at application domains where frames have been used with success. As with the previous material, we make no attempt to be exhaustive; we merely give a representative sample. These applications illustrate which basic properties of frames have found use in the real world. Redundancy is a major asset in many of these applications, as it is often used to gain stability, robustness, and resilience to noise. In some of the applications, frames have been used deliberately; by considering the requirements posed by applications, frames emerged as a natural choice. In others, only later have we become aware that the tools used were actually frames.

\subsection{Resilience to Noise}

It has been shown that frames show resilience to additive noise as well as numerical stability of reconstruction [64]. We start by illustrating resilience to noise.

Example 7.1 (Mercedes-Benz frame cont'd). We go back to our Mercedes-Benz frame and consider its unit-norm tight frame version 
given in (3.5). Suppose we perturb the frame coefficients by adding white noise $w_{i}$ to the channel $i$, where $E\left[w_{i}\right]=0, E\left[w_{i} w_{k}\right]=\sigma^{2} \delta_{i k}$ for $i, k=1,2,3$. We can now find the error of the reconstruction,

$$
x-\hat{x}=\frac{2}{3} \sum_{i=1}^{3}\left\langle x, \varphi_{i}\right\rangle \varphi_{i}-\frac{2}{3} \sum_{i=1}^{3}\left(\left\langle x, \varphi_{i}\right\rangle+w_{i}\right) \varphi_{i}=-\frac{2}{3} \sum_{i=1}^{3} w_{i} \varphi_{i} .
$$

Then the mean-squared error per component is

$$
\begin{aligned}
\mathrm{MSE} & =\frac{1}{2} E\|x-\hat{x}\|^{2}=\frac{1}{2} E\left\|\frac{2}{3} \sum_{i=1}^{3} w_{i} \varphi_{i}\right\|^{2} \\
& =\frac{1}{2} \sigma^{2} \frac{4}{9} \sum_{i=1}^{3}\left\|\varphi_{i}\right\|^{2}=\frac{2}{3} \sigma^{2},
\end{aligned}
$$

since all the frame vectors have norm 1 . Compare this to the same MSE obtained with an orthonormal basis: $\sigma^{2}$. In other words:

$$
\mathrm{MSE}_{\mathrm{ONB}}=\frac{3}{2} \mathrm{MSE}_{\mathrm{MB}}
$$

that is, the amount of error per component has been reduced using a frame.

Frames are thus generally considered to be robust under additive noise $[19,64,133]$. While modeling additive quantization noise as white is technically false and can be misleading [94], this example carries some useful intuition. Classical oversampled A/D conversion directly uses a harmonic tight frame and - when quantizer resolution is held fixed attains MSE inversely proportional to the oversampling factor. Other works in the area include [15, 18, 30, 60, 65, 93, 98]. Frames have been used with success in the analysis and optimization of $\Sigma \Delta$ quantization $[28,65,96]$ and oversampled A/D conversion where redundancy is used to gain robustness. Oversampled A/D conversion essentially corresponds to a tight frame expansion. An oversampled analog signal can be reconstructed by filters that have flat transfer characteristics in the frequency band occupied by the signal and arbitrary characteristics outside of that band, leading to the nonuniqueness of the reconstruction. Moreover, this allows for noise reduction upon reconstruction with 
the minimum norm dual, as the noise outside the signal's band is discarded, thus leading to the fact that the MSE behaves inversely proportional to the oversampling factor. Recently, the idea of beta-encodings has received significant attention in A/D conversion. Here again, the redundancy of these beta-expansions provides robustness with respect to quantizer imperfections. For more details, see [66]. In [97], Güntürk presents constructions of beta-encodings whose robustness to additive circuit noise is tunable.

\subsection{Compressive Sensing}

The field of compressive sensing has grown considerably over the past few years as attested by the recent special issue of the IEEE Signal Processing Magazine [135]. While not made explicit in the literature yet, compressive sensing theory seems to have strong ties to frame theory, and thus, we give here a brief overview. For more details, see tutorials by Candès et al. [37, 38] and Donoho [74].

Most objects or signals of interest are compressible, that is they can be encoded with just a few numbers without perceptual or numerical loss. Most acquisition, sensing and analog-to-digital protocols are dictated by Shannon's sampling theorem, and require the sampling rate to be at least twice the maximum frequency present in the signal (Nyquist rate). However, in most cases, only a small portion of the data acquired is actually used or important. That is where compressive sensing enters into play: It allows efficient and accurate signal acquisition by sensing and compressing simultaneously. The power of compressive sensing is that it uses much lower sampling rates than Nyquist to acquire signals and these sensing protocols are simple and signal independent. The signals are reconstructed using simple optimization algorithms. The main assumption made here is that the signals are compressible, that is, there exists an expansion in which they can be represented in a concentrated, compact way, or, in other words, a representation in which the signal is sparse. Based on this assumption, a compressive sensing process linearly correlates a signal $x \in \mathbb{R}^{n}$ (assumed to be sparse in some representation $\Psi$ ) with a fixed set of signal-independent atoms drawn from an overcomplete dictionary $\Phi$. 
The resulting linear measurements $y$ are then used in a simple convex optimization algorithm to recover the original signal $x$. The only condition on $\Phi$ is that it has to be "incoherent" with $\Psi$, that is, no element of $\Phi$ is sparse in $\Psi$ and vice versa. More formally, the compressive sensing problem can be formulated as follows: Assume $x$ is $k$-sparse in $\mathbb{R}^{n}$, that is, there exists a basis $\Psi$ of $\mathbb{R}^{n}$ such that $x=\Psi s$, where $s$ is an $n \times 1$ vector with $k$ nonzero coefficients. Let the vector $y$ of size $l \times 1(l<n)$ be the measurement vector computed through inner products between $x$ and the $n$ columns of the measurement (or sensing) matrix $\Phi$. Note that the sensing process is not adaptive, that is, $\Phi$ does not depend on $x$. Then one can write

$$
y=\Phi x=\Phi \Psi s .
$$

The compressive sensing problem consists of two parts: First, design a stable sensing incoherent matrix $\Phi$ such that it preserves all the salient information in any $k$-sparse vector despite the dimensionality reduction (from $\mathbb{R}^{n}$ to $\mathbb{R}^{l}$ ); and second, design a reconstruction algorithm to recover $x$ from the measurements $y$.

Since $l<n$, designing $\Phi$ is an ill-conditioned problem. However, when we assume $x$ is $k$-sparse and the $k$ locations in $s$ to be known, then the problem can be solved if $l \geq k$. A necessary and sufficient condition for the problem to be well-posed is that $\Omega=\Phi \Psi$ satisfies the so-called restricted isometry property [37]. The property states that for any vector $z$ that shares the same $k$ nonzero entries as $s$ and for some $\epsilon_{k}>0$

$$
\left(1-\epsilon_{k}\right)\|v\|_{2}^{2} \leq\|\Omega v\|_{2}^{2} \leq\left(1+\epsilon_{k}\right)\|v\|_{2}^{2},
$$

which means that $\Omega$ preserves the Euclidean norm of $k$-sparse vectors. An equivalent description of the restricted isometry property is to say that all subsets of $s$ columns of $\Omega$ are nearly orthogonal. We see that the restricted isometry property is very similar to saying that $\Omega$ is close to a Parseval tight frame. The restricted isometry property is important to ensure (and study) the robustness of compressive sensing protocols. In [75], Donoho et al. provide stability recovery results in the presence of noise. As for the optimization problem to reconstruct $x$, it can be posed as a linear program in $\ell^{1}$ and the restricted isometry 
property guarantees an accurate reconstruction. An emerging trend in compressive sensing seems to be random sensing, namely, choosing $\Phi$ as being generated from a random process, as this guarantees the restricted isometry property with very high probability. Randomness together with $\ell^{1}$ minimization lead to a near-optimal sensing protocol.

\subsection{Denoising}

Denoising with wavelets can be traced back to the work by Weaver et al. [170] (and even earlier to Witkin [172]), and was later on popularized by Donoho and Johnstone [73, 76]. Even then, sophisticated use of overcomplete expansions showed excellent results, and thus one of the first works on denoising with frames is [177], where the authors combined the overcomplete expansion with a variation of the technique from [129] to reconstruct the image from its wavelet maxima.

More recent works include cycle spinning introduced by Coifman and Donoho [53]. They suggest that when using a $j$-stage wavelet transform, one can take advantage of the fact that there are effectively $2^{j}$ different wavelet bases, each one corresponding to one of the $2^{j}$ shifts. Thus one can denoise in each of those $2^{j}$ wavelet bases and then average the result. Even though errors of individual estimates are generally positively correlated, one gets an advantage from averaging the estimators. Another effect of this is that the shift-varying basis gives way to a shiftinvariant frame (collection of bases). In [77], Dragotti et al. construct separable multidimensional directional frames for image denoising. The algorithm is in spirit similar to cycle spinning.

Ishwar and Moulin take a slightly different approach to develop a general framework for image modeling and estimation by fusing deterministic and statistical information from subband coefficients in multiple wavelet bases using maximum-entropy and set-theoretic ideas $[107,109,108,110]$. For instance, in [110] natural images are modeled as having sparse representations simultaneously in multiple orthonormal wavelet bases. Closed convex confidence tubes are designed around the wavelet coefficients of sparse initial estimates in multiple wavelet bases (frames). A projection onto convex sets algorithm is then used to arrive at a globally consistent sparse signal 
estimate. Denoising and restoration algorithms based on these image models produced visually sharper estimates with about 1-2 dB PSNR gains over competitive denoising algorithms such as the spatially adaptive Wiener filter.

Some other works include that by Fletcher et al. [88], where the authors analyze denoising by sparse approximation with frames. The known a priori information about the signal $x$ is that it has known sparsity $k$, that is, it can be represented via $k$ nonzero frame coefficients (with respect to a given frame $\Phi$ ). Then, after having been corrupted by noise yielding $\hat{X}$, the signal can be estimated by finding the best sparse approximation of $\hat{X}$. This work is essentially an attempt to understand how large a frame should be for denoising with a frame to be effective. In [50], the authors use the shift-invariant properties of the dual-tree $\mathbb{C W T}$ to provide better persistence across scale within the hidden Markov tree, and hence better denoising performance, while in [137], the steerable pyramid is used (see Section 6.6). An example of denoising by frames is given in Figure 7.1 (courtesy of Vivek Goyal).

\subsection{Resilience to Erasures}

Another application where frames found a natural home was that of robust transmission in communications. It was developed by Goyal et al. in [92], and was followed by works in [20, 21, 22, 25, 41, 105, 120, $139,157,165]$. The problem was that of creating multiple descriptions of the source so that when transmitted, and in the presence of losses, the source could be reconstructed based on received material. This clearly means that some amount of redundancy needs to be present in the system, since, if not, the loss of even one description would be irreversible.

In the initial work, the $\mathbb{R}^{n}$-valued source vector $x$ is represented through a frame expansion with frame a operator $\Phi^{*}$, yielding $X=$ $\Phi^{*} x \in \mathbb{R}^{m}$. The scalar quantization of the frame expansion coefficients gives $\hat{X}$ lying in a discrete subset of $\mathbb{R}^{m}$. One abstracts the effect of the network to be the erasure of some components of $\hat{X}$. This implies that the components of $\hat{X}$ are placed in more than one packet, for otherwise all of $\hat{X}$ could be lost at once. If they are placed in $m$ separate packets, 

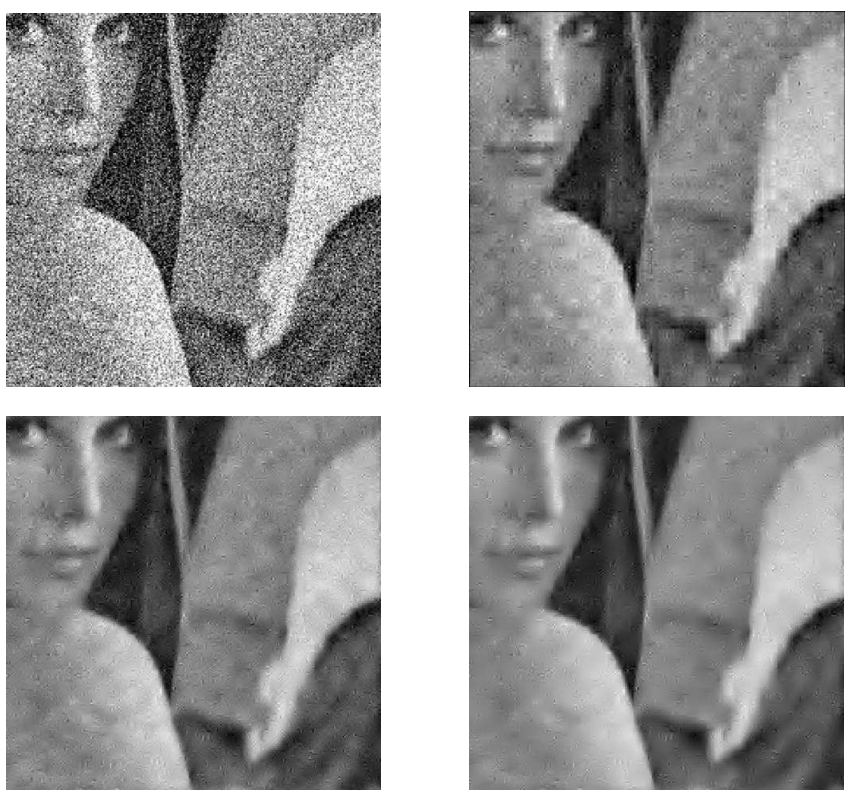

Fig. 7.1 Denoising with frames. Top left: Lena with $34.0 \mathrm{~dB}$ white Gaussian noise. Top right: Denoised Lena with $25.4 \mathrm{~dB}$ noise, using a soft threshold in a single basis. Bottom left: Denoised Lena with $24.2 \mathrm{~dB}$ noise, using cycle spinning (frame) from [53]. Bottom right: Denoised Lena with $23.2 \mathrm{~dB}$ noise, using differential cycle spinning (frame). The technique used here is an extension of the work in [87] (figure courtesy of Vivek Goyal).

then any subset of thecomponents of $\hat{X}$ may be received; otherwise only certain subsets are possible. The authors assume that linear reconstruction is used, that is, the dual frame is used to reconstruct. The authors model the noise as additive $\eta=\hat{X}-X$ as in Section 7.1 with the assumptions that every noise component is of zero mean and variance $\sigma^{2}$ and that they are uncorrelated. The canonical dual frame operator (3.10) is used as it minimizes the error of reconstruction. Losses in the network are modeled as erasures of a subset of quantized frame coefficients; to the decoder, it appears as if a quantized frame expansion were computed with the frame missing the elements which produced the erased ones, and thus, assuming it is a frame, a dual frame can be found. As a result, the authors concentrated on questions such as which deletions still leave a frame, which are the frames remaining frames under deletions of any subset of elements (up to $m-n$ ), etc. 
Example 7.2 (Mercedes-Benz frame cont'd). Assume now that one of the quantized coefficients in the Mercedes-Benz frame is lost, for example, $\hat{X}_{2}$. Does our frame have further nice properties when it comes to losses? First, that even with $\varphi_{2}$ not present, we can still use $\varphi_{1}$ and $\varphi_{3}$ to represent any vector in $\mathbb{R}^{2}$. The expansion formula is just not as elegant:

$$
x=\sum_{i=1,3}\left\langle x, \varphi_{i}\right\rangle \tilde{\varphi}_{i}
$$

with

$$
\tilde{\varphi}_{1}=\left(\begin{array}{c}
1 / \sqrt{3} \\
1
\end{array}\right), \quad \tilde{\varphi}_{3}=\left(\begin{array}{c}
2 / \sqrt{3} \\
0
\end{array}\right)
$$

Repeating the same calculations as those for the MSE in Section 7.1, we get that

$$
\begin{aligned}
\operatorname{MSE}_{\{2\}} & =\frac{1}{2} E\|x-\hat{x}\|^{2}=\frac{1}{2} E\left\|\sum_{i=1,3} w_{i} \tilde{\varphi}_{i}\right\|^{2} \\
& =\frac{1}{2} \sigma^{2} \sum_{i=1,3}\left\|\tilde{\varphi}_{i}\right\|^{2}=\frac{4}{3} \sigma^{2}
\end{aligned}
$$

that is, twice the MSE without erasures. However, the above calculation does not tell us anything about whether there is another frame with a lower MSE. In fact, given that one element is erased, does it really matter what the original frame was? It turns out that it does. In fact, among all frames with three norm- 1 frame vectors in $\mathbb{R}^{2}$, the MSE averaged over all possible erasures of one coefficient is minimized when the original frame is tight [92].

\subsection{Coding Theory}

While the set up is different, redundancy plays an important role in classical coding theory. For example, in convolutional coding theory, the encoding and decoding operations can formally be represented as passing the signal to be encoded through an oversampled analysis filter 
bank followed by an oversampled synthesis filter bank. In a seminal paper, Massey and Sain [132] give necessary and sufficient conditions for the existence of a FIR inverse (FIR synthesis filter bank) and the condition for which a convolutional code is catastrophic. They show that the nonuniqueness of the reconstruction can be exploited to make sure that the decoder (synthesis filter bank) does not lead to catastrophic error propagation.

Similar ideas can be found in error correcting codes where redundancy is used to buy robustness. Most notably, the early work of Wolf [173] shows that DFT codes are able to correct approximately twice as many errors as would have been predicted by the theory of these codes overs finite fields. ${ }^{1}$ In that work, the detection and correction of codes has been applied to impulse noise cancellation for pulse amplitude modulation transmission. More recently, Rath and Guillemot [140] clearly make the connection between frames and DFT codes by taking a frame-theoretic approach to study DFT codes with erasures. They show that DFT codes are a special class of frames and use lowpass DFT codes to provide robustness to channel erasures. More specifically, they prove that such tight frames produce the least reconstruction error. In addition, they define a subframe as a frame constructed from a subset of the frame vectors from an original frame (here, a DFT code) and derive packetization schemes thanks to their classification of subframes. Labeau et al. [123] used oversampled filter banks as error correcting codes and show the existence of a parity-check matrix for any given perfect reconstruction oversampled filter bank and give a spectral interpretation of the redundancy. They also outline the noise-correction capabilities of such filter banks.

\subsection{CDMA Systems}

The use of frames in CDMA systems dates back to Massey and Mittelholzer [131] on Welch bound and sequence sets for CDMA systems.

In a CDMA system, there are $m$ users who share the available spectrum. The sharing is achieved by "scrambling" $m$-dimensional user

\footnotetext{
${ }^{1}$ Note that DFT codes are cyclic codes over the complex field.
} 
vectors into smaller, $n$-dimensional vectors. In terms of frame theory, this scrambling corresponds to the application of a synthesis operator corresponding to $m$ distinct $n$-dimensional signature vectors $\varphi_{i}$ of norm $\sqrt{n}$. Noise-corrupted versions of these synthesized vectors arrive at a receiver, where the signature vectors are used to help extract the original user vectors. The variance of the interuser interference for user $i$ is

$$
\sigma_{i}^{2}=\sum_{\substack{j=1 \\ j \neq i}}^{m}\left|\left\langle\varphi_{i}, \varphi_{j}\right\rangle\right|^{2}-n^{2}
$$

leading to the total interuser interference:

$$
\sigma_{\text {tot }}^{2}=\sum_{i, j=1}^{m}\left|\left\langle\varphi_{i}, \varphi_{j}\right\rangle\right|^{2}-m n^{2}=\operatorname{FP}\left(\left\{\varphi_{i}\right\}_{i=1}^{m}\right)-m n^{2} .
$$

In the above, we recognize the frame potential from (4.6). The goal is to minimize the interferences and make them equal.

It is obvious that no interference is possible if and only if all $\varphi_{i}$ are orthogonal, and in turn, this is possible only if $m \leq n$, or, when $\varphi_{i}$ either form an orthogonal set or an orthonormal basis. When $m>n$, $\mathrm{FP}-m n^{2} \geq \mathrm{FP}-m^{2} n$ and the result is known as Welch bound ${ }^{2}$ : The sequences all have the same norm and

$$
\sum_{i, j=1}^{m}\left|\left\langle\varphi_{i}, \varphi_{j}\right\rangle\right|^{2} \geq m^{2} n
$$

with equality if and only if the $m \times n$ matrix $\Phi^{*}$ whose rows are $\varphi_{i}^{*}$ has orthogonal columns of norm $\sqrt{n}$. If we normalize every vector to be unit norm, we can immediately translate the above into frame parlance (see Theorem 4.2): (a) Welch bound is equivalent to the frame potential inequality. (b) Frame potential is minimized at tight frames. (c) $m \times n$ matrix is the analysis operator $\Phi^{*}$. (d) Columns of the analysis operator of a tight frame are orthogonal (consequence of the Naimark theorem).

2 The question of which expression is the actual Welch bound frequently leads to confusion. In his original paper [171], Welch found the lower bound on the maximum value of the cross-correlation of complex sequences, given in (6.4). In 1992, Massey and Mittelholzer [131] rephrased it in terms of the bound on the maximum user interference as given in (7.4). 
This work was followed by many others, among those, by Viswanath and Anantharam's [168] discovery of the fundamental inequality (4.7) during their investigation of the capacity region in synchronous CDMA systems. The authors showed that the design of the optimal signature matrix $S$ depends upon the powers $\left\{p_{i}=\left\|\varphi_{i}\right\|^{2}\right\}_{i=1}^{m}$ of the individual users. In particular, they divided the users into two classes: those that are oversized and those that are not. While the oversized users are assigned orthogonal channels for their personal use, the remaining users have their signature vectors designed so as to be Welch bound equality sequences, namely, sequences which achieve the lower bound for the frame potential, and are thus tight frames (see Theorem 4.2).

When no user is oversized, that is, when the fundamental inequality is satisfied, their problem reduces to finding a tight frame for $\mathbb{H}$

with norms $\left\{\sqrt{p_{i}}\right\}_{i=1}^{m}$. The authors gave one solution to the problem using an explicit construction; characterization of all solutions to this problem using a physical interpretation of frame theory was given in Theorem 4.3 [40], Section 4.

The equivalence between unit-norm tight frames and Welch bound sequences was shown in [157]. Waldron formalized that equivalence for general tight frames in [169], and consequently, tight frames have been referred in some works as Welch bound sequences [161].

\subsection{Multiantenna Code Design}

An important application of equal-norm Parseval tight frames is in multiple-antenna code design [100]. Much theoretical work has been done to show that communication systems which employ multiple antennas can have very high channel capacities [89, 159]. These methods rely on the assumption that the receiver knows the complex-valued Rayleigh fading coefficients. To remove this assumption, in [104] new classes of unitary space-time signals are proposed. If we have $n$ transmitting antennas and we transmit in blocks of $m$ time samples (over which the fading coefficients are approximately constant), then a constellation of $K$ unitary space-time signals is a (weighted by $\sqrt{m}$ ) collection of $n \times m$ complex matrices $\left\{\Phi_{k}\right\}$ for which $\Phi_{k} \Phi^{*}{ }_{k}=I$, a Parseval tight frame in other words. The $i$ th row of any $\Phi_{k}$ contains the signal 
transmitted on antenna $i$ as a function of time. The only structure required in general is the time-orthogonality of the signals.

Originally it was believed that designing such constellations was a too cumbersome and difficult optimization problem for practice. However, in [104], it was shown that constellations arising in a "systematic" fashion can be done with relatively little effort. Systematic here means that we need to design high-rate space-time constellations with low encoding and decoding complexity. It is known that full transmitter diversity (that is, where the constellation is a set of unitary matrices whose differences have nonzero determinant) is a desirable property for good performance. In a tour-de-force, in [100], the authors used fixedpoint-free groups and their representations to design high-rate constellations with full diversity. Moreover, they classified all full-diversity constellations that form a group, for all rates and numbers of transmitting antennas.

\subsection{From Biology to Teleportation}

We now briefly touch upon a host of other applications from standard to fairly esoteric ones such as quantum teleportation.

Frames were used for compression in the 1980s by Burt and Adelson who proposed pyramid coding of images [33] which used redundant linear transforms and was quite successful for a while (see Section 6.6.1).

If one considers the segmentation problem as classification into object and background, the work of $[162,124]$ then uses frames for segmentation. In a more recent work, de Rivaz and Kingsbury use the dual-tree $\mathbb{C W T}$ (see Section 6.5.1) to formulate the energy terms for the level-set based active contour segmentation approach [69]. They use a limited redundancy transform with a fast implementation. Both Laine and Unser used frames to decompose textures in order to characterize them across scales [124, 162]. In [47] the authors use frames for image interpolation and resolution enhancement. In [48], the authors use frames to significantly improve the classification accuracy of protein subcellular location images, to close to $96 \%$ as well as the high-throughput tagging of Drosophila embryo developmental stages [112]. In theoretical neuroscience, another advantage of frames 
has been presented in [134] where the authors argue that overcomplete representations are probably necessary in the simple cells of mammalian visual cortex and shed light onto some cortical processing at that level.

Regularized inversion problems such as deblurring in noise can also greatly benefit from the ability of redundant frames to provide signal models that allow Bayesian regularization constraints to be applied efficiently to complicated signals such as images, as illustrated in [70].

Another application of frames has been in signal reconstruction from nonuniform samples, see $[3,12,85]$ and references therein. In [36], Candès and Donoho provide an example of tight frames that outperform any known basis for approximation purposes. Benedetto and Pfander used redundant wavelet transforms (frames) to predict epileptic seizures $[16,17]$. Kingsbury used his dual-tree $\mathbb{C W T}$ for restoration and enhancement [113], motion estimation [128] as well as building feature, texture and object detectors for images [4, 84, 116]. In [130], Malvar demonstrates that the modulated complex lapped transform is well suited for noise suppression and echo cancellation, whereas in [178], it is advantageously used for audio coding. In [152], Šoljanin makes the connection between harmonic tight frames and network coding. Xia, among others, shows how oversampled filter banks can be used in precoding and equalization for intersymbol interference cancellation [176]. Balan et al. used frames for signal reconstruction without noisy phase within speech recognition problems [6]. Many connections have been made between frames and coding theory [101, 143].

Recently, certain quantum measurement results have been recast in terms of frames $[80,81,151,153]$. They have applications in quantum computing and have to do with positive operator valued measures. Who knows, maybe Star Trek comes to life, and frames play a role in quantum teleportation [31]! 


\section{Conclusions}

Coming to the end of this survey, we hope you have a different picture of a frame in your mind from a "picture frame." While necessarily colored by our personal bias, we intended this survey as a basic introduction to frames, geared primarily toward engineering students and those without extensive mathematical training. Frames are here to stay; as wavelet bases before them, they are becoming a standard tool in the signal processing toolbox, spurred by a host of recent applications requiring some level of redundancy. We hope this survey will be of help when deciding whether frames are the right tool for your application. 


\section{Nomenclature and Notation}

Frame nomenclature is far from uniform and can result in confusion. For example, frames with unit-norm frame vectors have been called normalized frames (normalized as in all vectors normalized to norm 1, similarly to the meaning of normalized in orthonormal bases), uniform, as well as uniform frames with norm 1. The names of various classes of frames, as well as alternate names under

Table A.1 Frame nomenclature.

\begin{tabular}{|c|c|c|}
\hline Name & Description & Alternate Names \\
\hline Equal-norm frame & $\begin{array}{l}\left\|\varphi_{i}\right\|=\left\|\varphi_{j}\right\| \\
\text { for all } i, j\end{array}$ & Uniform frame [119] \\
\hline Unit-norm frame & $\begin{array}{l}\left\|\varphi_{i}\right\|=1 \\
\text { for all } i\end{array}$ & $\begin{array}{l}\text { Uniform frame with norm } 1 \text { [119] } \\
\text { Uniform frame [92] } \\
\text { Normalized frame [13] }\end{array}$ \\
\hline Tight frame & $A=B$ & $A$-tight frame \\
\hline Parseval tight frame & $A=B=1$ & Normalized frame [7] \\
\hline Unit-norm tight frame & $\begin{array}{l}A=B \\
\left\|\varphi_{i}\right\|=1 \\
\text { for all } i\end{array}$ & $\begin{array}{l}\text { Uniform tight frame with norm } 1 \text { [119] } \\
\text { Uniform tight frame [92] } \\
\text { Normalized tight frame [13] }\end{array}$ \\
\hline
\end{tabular}




\section{Nomenclature and Notation}

which they have been used in the literature, are given in Table A.1, while Figure 3.3 shows those same classes of frames and their relationships. Frame notation is given in Table A.2, while various frame properties are given in Table A.3.

Table A.2 Frame notation.

\begin{tabular}{lll}
\hline Symbol & & Explanation \\
\hline $\mathbb{H}=$ & $\mathbb{R}^{n}$ & Real Hilbert space \\
& $\mathbb{C}^{n}$ & Complex Hilbert space \\
& $\ell^{2}(\mathbb{Z})$ & Space of square-summable sequences \\
$I=$ & $\{1, \ldots, m\}$ & Index set for $\mathbb{R}^{n}, \mathbb{C}^{n}$ \\
& $\mathbb{Z}$ & Index set for $\ell^{2}(\mathbb{Z})$ \\
When $\mathbb{H}=\mathbb{R}^{n}, \mathbb{C}^{n}$ & $n$ & Dimension of the space \\
& $m$ & Number of frame vectors \\
$\varphi_{i} \in \mathbb{H}$ & & Frame vector \\
$\Phi=\left\{\varphi_{i}\right\}_{i \in I}$ & & Frame family \\
$\Phi^{*}$ & & Analysis operator \\
$S=$ & $\Phi \Phi^{*}$ & Frame operator \\
$G=$ & $\Phi^{*} \Phi$ & Grammian \\
$\tilde{\varphi}_{i} \in \mathbb{H}$ & $S^{-1} \varphi_{i}$ & Dual frame vector \\
$\tilde{\Phi}=\left\{\tilde{\varphi}_{i}\right\}_{i \in I}$ & & Dual frame family \\
$\tilde{\Phi}^{*}=$ & $\Phi^{*} S^{-1}$ & Dual analysis operator \\
$\tilde{S}=$ & $S^{-1}$ & Dual frame operator \\
$\tilde{G}=$ & $\Phi^{*} S^{-2} \Phi$ & Dual Grammian \\
\hline
\end{tabular}


Table A.3 Summary of properties for various classes of frames. All trace identities are given for $\mathbb{H}=\mathbb{R}^{n}, \mathbb{C}^{n}$.

\begin{tabular}{|c|c|c|}
\hline Frame & Constraints & Properties \\
\hline General & $\begin{array}{l}\left\{\varphi_{i}\right\}_{i \in I} \\
\text { is a Riesz basis } \\
\text { for } \mathbb{H}\end{array}$ & $\begin{array}{l}A\|x\|^{2} \leq \sum_{i \in I}\left|\left\langle x, \varphi_{i}\right\rangle\right|^{2} \leq B\|x\|^{2} \\
A I \leq S \leq B I \\
\operatorname{tr}(S)=\sum_{j=1}^{n} \lambda_{j}=\operatorname{tr}(G)=\sum_{i=1}^{m}\left\|\varphi_{i}\right\|^{2}\end{array}$ \\
\hline $\begin{array}{l}\text { Equal-norm } \\
\text { frame }\end{array}$ & $\begin{array}{l}\left\|\varphi_{i}\right\|=\left\|\varphi_{j}\right\|=a \\
\text { for all } i \text { and } j\end{array}$ & $\begin{array}{l}A\|x\|^{2} \leq \sum_{i \in I}\left|\left\langle x, \varphi_{i}\right\rangle\right|^{2} \leq B\|x\|^{2} \\
A I \leq S \leq B I \\
\operatorname{tr}(S)=\sum_{j=1}^{n} \lambda_{j}=\operatorname{tr}(G)=\sum_{i=1}^{m}\left\|\varphi_{i}\right\|^{2}=m a^{2}\end{array}$ \\
\hline Tight frame & $A=B$ & $\begin{array}{l}\sum_{i \in I}\left|\left\langle x, \varphi_{i}\right\rangle\right|^{2}=A\|x\|^{2} \\
S=A I \\
\operatorname{tr}(S)=\sum_{j=1}^{n} \lambda_{j}=n A=\operatorname{tr}(G)=\sum_{i=1}^{m}\left\|\varphi_{i}\right\|^{2}\end{array}$ \\
\hline $\begin{array}{l}\text { Parseval } \\
\text { tight frame }\end{array}$ & $A=B=1$ & $\begin{array}{l}\sum_{i \in I}\left|\left\langle x, \varphi_{i}\right\rangle\right|^{2}=\|x\|^{2} \\
S=I \\
\operatorname{tr}(S)=\sum_{j=1}^{n} \lambda_{j}=n=\operatorname{tr}(G)=\sum_{i=1}^{m}\left\|\varphi_{i}\right\|^{2}\end{array}$ \\
\hline $\begin{array}{l}\text { Equal-norm } \\
\text { tight frame }\end{array}$ & $\begin{array}{l}A=B \\
\left\|\varphi_{i}\right\|=\left\|\varphi_{j}\right\|=a \\
\text { for all } i \text { and } j\end{array}$ & $\begin{array}{l}\sum_{i \in I}\left|\left\langle x, \varphi_{i}\right\rangle\right|^{2}=A\|x\|^{2} \\
S=A I \\
\operatorname{tr}(S)=\sum_{j=1}^{n} \lambda_{j}=n A=\operatorname{tr}(G)=\sum_{i=1}^{m}\left\|\varphi_{i}\right\|^{2}=m a^{2} \\
A=(m / n) a^{2}\end{array}$ \\
\hline $\begin{array}{l}\text { Unit-norm } \\
\text { tight frame }\end{array}$ & $\begin{array}{l}A=B \\
\left\|\varphi_{i}\right\|=1 \\
\text { for all } i\end{array}$ & $\begin{array}{l}\sum_{i \in I}\left|\left\langle x, \varphi_{i}\right\rangle\right|^{2}=A\|x\|^{2} \\
S=A I \\
\operatorname{tr}(S)=\sum_{j=1}^{n} \lambda_{j}=n A=\operatorname{tr}(G)=\sum_{i=1}^{m}\left\|\varphi_{i}\right\|^{2}=m \\
A=m / n\end{array}$ \\
\hline $\begin{array}{l}\text { Equal-norm } \\
\text { Parseval } \\
\text { tight frame }\end{array}$ & $\begin{array}{l}A=B=1 \\
\left\|\varphi_{i}\right\|=\left\|\varphi_{j}\right\|=a \\
\text { for all } i \text { and } j\end{array}$ & $\begin{array}{l}\sum_{i \in I}\left|\left\langle x, \varphi_{i}\right\rangle\right|^{2}=\|x\|^{2} \\
S=I \\
\operatorname{tr}(S)=\sum_{j=1}^{n} \lambda_{j}=n=\operatorname{tr}(G)=\sum_{i=1}^{m}\left\|\varphi_{i}\right\|^{2}=m a^{2} \\
a=\sqrt{n / m}\end{array}$ \\
\hline $\begin{array}{l}\text { Unit-norm } \\
\text { Parseval } \\
\text { tight frame } \\
\Leftrightarrow \\
\text { Orthonormal } \\
\text { basis }\end{array}$ & $\begin{array}{l}A=B=1 \\
\left\|\varphi_{i}\right\|=1 \\
\text { for all } i\end{array}$ & $\begin{array}{l}\sum_{i \in I}\left|\left\langle x, \varphi_{i}\right\rangle\right|^{2}=\|x\|^{2} \\
S=I \\
\operatorname{tr}(S)=\sum_{j=1}^{n} \lambda_{j}=n=\operatorname{tr}(G)=\sum_{i=1}^{m}\left\|\varphi_{i}\right\|^{2}=m \\
n=m\end{array}$ \\
\hline
\end{tabular}




\section{Acknowledgments}

We gratefully acknowledge comments from anonymous reviewers, their time and effort hopefully resulted in a better survey. Our frame community has come through with numerous comments: Riccardo Bernardini, Bernhard Bodmann, Helmut Bölcskei, Pete Casazza, Minh Do, Matt Fickus, Prakash Ishwar, Nick Kingsbury, Götz Pfander, Emina Šljanin, Thomas Strohmer, and Martin Vetterli; their collective wisdom improved the original survey significantly. In particular, Chris Heil's and Zoran Cvetković's help was invaluable in making the paper mathematically tight and precise. We are also indebted to Vivek Goyal for providing us with Figure 7.1 and helping with questions both along the way and very last minute. James Finlay has been infinitely patient while keeping just the right amount of pressure. We thank Bob Gray for his support and encouragement, as well as an extremely thorough review. 


\section{References}

[1] A. F. Abdelnour and I. W. Selesnick, "Symmetric nearly shift-invariant tight frame wavelets," IEEE Transactions on Signal Processing, vol. 53, no. 1, pp. 231-239, January 2005.

[2] N. I. Akhiezer and I. M. Glazman, Theory of Linear Operators in Hilbert Spaces. Vol. 1, Frederick Ungar, 1966.

[3] A. Aldroubi and K. Gröchenig, "Nonuniform sampling and reconstruction in shift-invariant spaces," SIAM Review, vol. 43, no. 4, pp. 585-620, 2001.

[4] R. Anderson, N. G. Kingsbury, and J. Fauqueur, "Rotation-invariant object recognition using edge-profile clusters," Proceedings of European Conference on Signal Processing, September 2006.

[5] P. Auscher, "Remarks on the local Fourier bases," Wavelets: Mathematics and Applications, pp. 203-218, CRC Press, 1994.

[6] R. Balan, P. G. Casazza, and D. Edidin, "On signal reconstruction without phase," Journal on Applied and Computational Harmonic Analysis, vol. 20, no. 3, pp. 345-356, 2006.

[7] R. Balan, P. G. Casazza, C. Heil, and Z. Landau, "Deficits and excesses of frames," Advances in Computational Mathematics, vol. 18, pp. 93-116, 2003.

[8] R. Balan, P. G. Casazza, C. Heil, and Z. Landau, "Density, overcompleteness, and localization of frames, I. Theory," Journal of Fourier Analysis and Applications, vol. 12, pp. 105-143, 2006.

[9] R. Balan, P. G. Casazza, C. Heil, and Z. Landau, "Density, overcompleteness, and localization of frames, II. Gabor frames," Journal of Fourier Analysis and Applications, vol. 12, pp. 307-344, 2006. 


\section{References}

[10] I. Bayram and I. W. Selesnick, "Overcomplete discrete wavelet transforms with rational dilation factors," IEEE Transactions on Signal Processing, accepted, 2008.

[11] M. G. Bellanger and J. L. Daguet, "TDM-FDM transmultiplexer: Digital polyphase and FFT," IEEE Transactions on Communications, vol. 22, no. 9, pp. 1199-1204, September 1974.

[12] J. J. Benedetto, "Irregular sampling and frames," in Wavelets: A Tutorial in Theory and Applications, pp. 445-507, Academic Press, 1992.

[13] J. J. Benedetto and M. C. Fickus, "Finite normalized tight frames," Advances in Computational Mathematics, Frames, vol. 18, pp. 357-385, (special issue) 2003.

[14] J. J. Benedetto and A. Kebo, "The role of frame force in quantum detection," Journal on Fourier Analysis and Applications, vol. 14, pp. 443-474, 2008.

[15] J. J. Benedetto and O. Oktay, "PCM-sigma delta comparison and sparse representation quantization," in Proceedings of CISS, Princeton, NJ, March 2008.

[16] J. J. Benedetto and G. E. Pfander, "Wavelet periodicity detection algorithms," in Proceedings of SPIE Conference on Wavelet Applications in Signal and Image Processing, pp. 48-55, San Diego, CA, July 1998.

[17] J. J. Benedetto and G. E. Pfander, "Periodic wavelet transforms and periodicity detection," SIAM Journal on Applied Mathematics, vol. 62, pp. 1329-1368, 2002.

[18] J. J. Benedetto, A. M. Powell, and Ö. Yilmaz, "Sigma-delta quantization and finite frames," IEEE Transactions on Information Theory, vol. 52, pp. 19902005, 2006.

[19] J. J. Benedetto and O. Treiber, "Wavelet frames: Multiresolution analysis and extension principles," in Wavelet Transforms and Time-Frequency Signal Analysis, Birkhäuser, 2001.

[20] R. Bernardini and R. Rinaldo, "Efficient reconstruction from frame-based multiple descriptions," IEEE Transactions on Signal Processing, vol. 53, no. 8, pp. 3282-3296, August 2005.

[21] R. Bernardini and R. Rinaldo, "Bounds on error amplification in oversampled filter banks for robust transmission," IEEE Transactions on Signal Processing, vol. 54, no. 4, pp. 1399-1411, April 2006.

[22] R. Bernardini and R. Rinaldo, "Oversampled filter banks from extended perfect reconstruction filter banks," IEEE Transactions on Signal Processing, vol. 54, no. 7, July 2006.

[23] B. G. Bodmann, "Optimal linear transmission by loss-insensitive packet encoding," Journal on Applied and Computational Harmonic Analysis, vol. 22, no. 3, pp. 274-285, 2007.

[24] B. G. Bodmann, P. G. Casazza, D. Edidin, and R. Balan, "Frames for linear reconstruction without phase," in Proceedings of CISS, Princeton, NJ, March 2008.

[25] B. G. Bodmann and V. I. Paulsen, "Frames, graphs and erasures," Linear Algebra and Its Applications, pp. 118-146, 2005.

[26] H. Bölcskei and F. Hlawatsch, "Oversampled modulated filter banks," in Gabor Analysis and Algorithms: Theory and Applications, pp. 295-322, Boston, MA: Birkhäuser, 1998. 
[27] H. Bölcskei and F. Hlawatsch, "Oversampled cosine modulated filter banks with perfect reconstruction," IEEE Transactions on Circle and Systems II: Analog and Digital Signal Processing, vol. 45, no. 8, pp. 1057-1071, August 1998.

[28] H. Bölcskei and F. Hlawatsch, "Noise reduction in oversampled filter banks using predictive quantization," IEEE Transactions on Information Theory, vol. 47, no. 1, pp. 155-172, January 2001.

[29] H. Bölcskei, F. Hlawatsch, and H. G. Feichtinger, "Frame-theoretic analysis of oversampled filter banks," IEEE Transactions on Signal Processing, vol. 46, no. 12 , pp. 3256-3269, December 1998.

[30] S. Borodachov and Y. Wang, "Asymptotic white noise hypothesis for PCM quantization," in Proceedings of CISS, Princeton, NJ, March 2008.

[31] D. Bouwmeester, J.-W. Pan, K. Mattle, M. Eibl, H. Weinfurter, and A. Zeilinger, "Experimental quantum teleportation," Nature, vol. 390, p. 575, December 1997.

[32] A. P. Bradley, "Shift invariance in the discrete wavelet transform," in Proceedings of Digital Image Computing, December 2003.

[33] P. J. Burt and E. H. Adelson, "The Laplacian pyramid as a compact image code," IEEE Transactions on Communications, vol. 31, no. 4, pp. 532-540, April 1983.

[34] E. J. Candès, Curvelet Web Site. http://www.curvelet.org/papers.html.

[35] E. J. Candès, L. Demanet, D. L. Donoho, and L. Ying, "Fast discrete curvelet transforms," SIAM Multiscale Model Simulation, vol. 5, no. 3, pp. 861-899, 2006.

[36] E. J. Candès and D. L. Donoho, "New tight frames of curvelets and optimal representation of objects with piecewise C2 singularities," Communications on Pure and Applied Mathematics, vol. 57, pp. 219-266, 2004.

[37] E. J. Candès, J. Romberg, and T. Tao, "Robust uncertainty principles: Exact signal reconstruction from highly incomplete frequency information," IEEE Transactions on Information Theory, vol. 52, no. 2, pp. 489-509, February 2006.

[38] E. J. Candès and T. Tao "Near optimal signal recovery from random projections: Universal encoding strategies?" IEEE Transactions on Information Theory, vol. 52, no. 12, pp. 5406-5425, December 2006.

[39] P. G. Casazza, "The art of frame theory," Taiwanese Journal on Mathematics, vol. 4, no. 2, pp. 129-202, 2000.

[40] P. G. Casazza, M. C. Fickus, J. Kovačević, M. Leon, and J. C. Tremain, "A physical interpretation for finite tight frames," in Harmonic Analysis and Applications, pp. 51-76, Boston, MA: Birkhäuser, 2006.

[41] P. G. Casazza and J. Kovačević, "Equal-norm tight frames with erasures," Advances on Computational Mathematics, Frames, vol. 18, pp. 387-430, (special issue), 2002.

[42] P. G. Casazza and G. Kutyniok, "Frames of subspaces," Contemporary Mathematics, vol. 345, pp. 87-113, 2004.

[43] P. G. Casazza and G. Kutyniok, "Robustness of fusion frames under erasures of subspaces and of local frame vectors," in Radon Transforms, Geometry, and Wavelets, (E. L. Grinberg, D. Larson, P. E. T. Jorgensen, P. Massopust, 
G. Olafsson, E. T. Quinto, and B. Rubi, eds.), Contemporary Mathematics, Amer. Math. Soc., Providence, RI, to appear, 2008.

[44] P. G. Casazza, G. Kutyniok, and S. Li, "Fusion frames and distributed processing," Journal on Applied and Computational Harmonic Analysis, vol. 25, pp. 114-132, 2008.

[45] P. G. Casazza, G. Kutyniok, S. Li, and A. Pezeshki, Fusion Frames. http://www.fusionframe.org/.

[46] P. G. Casazza and N. Leonhard, "The known equal norm Parseval frames as of 2005," Technical Report, University of Missouri, 2005.

[47] S. G. Chang, Z. Cvetković, and M. Vetterli, "Resolution enhancement of images using wavelet extrema extrapolation," in Proceedings of IEEE International Conference on Acoustics, Speech and Signal Processing, vol. 4, pp. 23792382, Detroit, MI, May 1995.

[48] A. Chebira, Y. Barbotin, C. Jackson, T. E. Merryman, G. Srinivasa, R. F. Murphy, and J. Kovačević "A multiresolution approach to automated classification of protein subcellular location images," BMC Bioinformatics, vol. 8, no. 210, 2007, http://www.andrew.cmu.edu/user/jelenak/Repository/ 07_ChebiraBJMSMK/07_ChebiraBJMSMK.html.

[49] A. Chebira and J. Kovačević, "Lapped tight frame transforms," in Proceedings of IEEE International Conference Acoustics, Speech and Signal Processing, vol. III, pp. 857-860, Honolulu, HI, April 2007.

[50] H. Choi, J. Romberg, R. Baraniuk, and N. G. Kingsbury, "Hidden Markov tree modelling of complex wavelet transforms," in Proceedings of IEEE International Conference Acoustics, Speech and Signal Processing, Istanbul, Turkey, June 2000.

[51] O. Christensen, An Introduction to Frames and Riesz Bases. Birkhäuser, 2002.

[52] O. Christensen and Y. C. Eldar, "Oblique dual frames and shift-invariant spaces," Journal on Applied and Computational Harmonic Analysis, vol. 17, no. 1, pp. 48-68, July 2004.

[53] R. R. Coifman and D. L. Donoho, "Translation-invariant denoising," in Wavelets and Statistics, New York, NY: Springer-Verlag, 1995.

[54] R. R. Coifman, Y. Meyer, S. Quake, and M. V. Wickerhauser, "Signal processing and compression with wavelet packets," Technical Report, Yale University, 1991.

[55] J. Conway, R. H. Hardin, and N. J. A. Sloane, "Packing lines, planes, etc: Packings in Grassmanian spaces," Experimental Mathematics, vol. 5, no. 2, pp. 139-159, 1996.

[56] J. Conway and N. J. A. Sloane, Sphere Packings, Lattices and Groups. Springer-Verlag, 1992.

[57] A. Croisier, D. Esteban, and C. Galand, "Perfect channel splitting by use of interpolation/decimation/tree decomposition techniques," in Proceedings of International Conference on Information Sciences and System, pp. 443-446, Patras, Greece, August 1976.

[58] A. L. Cunha, J. Zhou, and M. N. Do, "The nonsubsampled contourlet transform: Theory, design, and applications," IEEE Transactions on Image Processing, vol. 15, no. 10, pp. 3089-3101, October 2006. 
[59] Z. Cvetković, "Oversampled modulated filter banks and tight Gabor frames in $\ell^{2}(\mathbb{Z})$," in Proceedings of IEEE International Conference on Acoustics, Speech and Signal Processing, pp. 1456-1459, Detroit, MI, May 1995.

[60] Z. Cvetković, "Resilience properties of redundant expansions under additive noise and quantization," IEEE Transactions on Information Theory, vol. 49, no. 3, pp. 644-656, March 2003.

[61] Z. Cvetković and M. Vetterli, "Oversampled filter banks," IEEE Transactions on Signal Processing, vol. 46, no. 5, pp. 1245-1255, May 1998.

[62] Z. Cvetković and M. Vetterli, "Tight Weyl-Heisenberg frames in $\ell^{2}(\mathbb{Z})$," IEEE Transactions on Signal Processing, vol. 46, no. 5, pp. 1256-1259, May 1998.

[63] I. Daubechies, "The wavelet transform, time-frequency localization and signal analysis," IEEE Transactions on Information Theory, vol. 36, no. 5, pp. 9611005, September 1990.

[64] I. Daubechies, Ten Lectures on Wavelets. Philadelphia, PA: SIAM, 1992.

[65] I. Daubechies and R. DeVore, "Reconstructing a bandlimited function from very coarsely quantized data: A family of stable sigma-delta modulators of arbitrary order," Annals of Mathematics, vol. 158, no. 2, pp. 679-710, 2003.

[66] I. Daubechies, R. A. DeVore, C. S. Güntürk, and V. A. Vaishampayan, "A/D conversion with imperfect quantizers," IEEE Transactions on Information Theory, vol. 52, no. 3, pp. 874-885, March 2006.

[67] I. Daubechies, A. Grossman, and Y. Meyer, "Painless nonorthogonal expansions," Journal on Mathematical Physics, vol. 27, pp. 1271-1283, November 1986.

[68] I. Daubechies, H. J. Landau, and Z. Landau, "Gabor time-frequency lattices and the Wexler-Raz identiry," Journal on Fourier Analysis and Applications, vol. 1, no. 4, pp. 437-478, November 1994.

[69] P. de Rivaz and N. G. Kingsbury, "Fast segmentation using level set curves of complex wavelet surfaces," in Proceedings of IEEE International Conference on Image Processing, Vancouver, Canada, September 1998.

[70] P. F. C. de Rivaz and N. G. Kingsbury, "Bayesian image deconvolution and denoising using complex wavelets," in Proceedings of IEEE International Conference on Image Processing, Thessaloniki, Greece, October 2001.

[71] M. N. Do and M. Vetterli, "Framing pyramids," IEEE Transactions on Signal Processing, vol. 51, no. 9, pp. 2329-2342, September 2003.

[72] M. N. Do and M. Vetterli, "The contourlet transform: An efficient directional multiresolution image representation," IEEE Transactions on Image Processing, vol. 14, no. 12, pp. 2091-2106, December 2005.

[73] D. L. Donoho, "De-noising by soft-thresholding," IEEE Transactions on Information Theory, vol. 41, pp. 613-627, March 1995.

[74] D. L. Donoho, "Compressed sensing," IEEE Transactions on Information Theory, vol. 52, no. 4, pp. 1289-1306, April 2006.

[75] D. L. Donoho, M. Elad, and V. N. Temlyakov, "Stable recovery of sparse overcomplete representations in the presence of noise," IEEE Transactions on Information Theory, vol. 52, no. 1, pp. 6-18, January 2006.

[76] D. L. Donoho and I. M. Johnstone, "Ideal spatial adaptation via wavelet shrinkage," Biometrika, vol. 81, pp. 425-455, 1994. 
[77] P. L. Dragotti, V. Velisavljević, M. Vetterli, and B. Beferull-Lozano, "Discrete directional wavelet bases and frames for image compression and denoising," in Proceedings of SPIE Conference on Wavelet Applications in Signal and Image Processing, August 2003.

[78] R. J. Duffin and A. C. Schaeffer, "A class of nonharmonic Fourier series," Transactions on American Mathematical Society, vol. 72, pp. 341-366, 1952.

[79] Y. Eldar and H. Bölcskei, "Geometrically uniform frames," IEEE Transactions on Information Theory, vol. 49, no. 4, pp. 993-1006, April 2003.

[80] Y. Eldar and G. Forney, Jr., "Optimal tight frames and quantum measurement," IEEE Transactions on Information Theory, vol. 48, no. 3, pp. 599-610, March 2002.

[81] N. Elron and Y. C. Eldar, "Optimal encoding of classical information in a quantum medium," IEEE Transactions on Information Theory, vol. 53, no. 5, pp. 1900-1907, May 2007.

[82] B.-G. Englert, 2004, http://www.imaph.tu-bs.de/qi/problems/13.html.

[83] D. Esteban and C. Galand, "Applications of quadrature mirror filters to split band voice coding schemes," in Proceedings of IEEE International Conference on Acoustics, Speech and Signal Processing, pp. 191-195, 1995.

[84] J. Fauqueur, N. G. Kingsbury, and R. Anderson, "Multiscale keypoint detection using the dual-tree complex wavelet transform," Proceedings of IEEE International Conference on Image Processing, October 2006.

[85] H. G. Feichtinger and K. Gröchenig, "Theory and practice of irregular sampling," Wavelets: Mathematics and Applications, pp. 305-363, CRC Press, 1994.

[86] H. G. Feichtinger and T. Strohmer, eds., Gabor Analysis and Algorithms: Theory and Applications. Boston, MA: Birkhäuser, 1998.

[87] A. K. Fletcher, V. K. Goyal, and K. Ramchandran, "Iterative projective wavelet methods for denoising," in Proceedings of SPIE Conference on Wavelet Applications in Signal and Image Processing, vol. 5207, pp. 9-15, San Diego, CA, August 2003.

[88] A. K. Fletcher, S. Rangan, V. K. Goyal, and K. Ramchandran, "Denoising by sparse approximation: Error bounds based on rate-distortion theory," EURASIP Journal on Applied Signal Processing, pp. 1-19, March 2006.

[89] G. J. Foschini and M. J. Gans, "On the limits of wireless communications in a fading environment when using multiple antennas," Wireless Personal Communications, vol. 6, no. 3, pp. 311-335, March 1998.

[90] D. Gabor, "Theory of communication," Journal of IEE, vol. 93, pp. 429-457, 1946.

[91] I. Gohberg and S. Goldberg, Basic Operator Theory. Boston, MA: Birkhäuser, 1981.

[92] V. K. Goyal, J. Kovačević, and J. A. Kelner, "Quantized frame expansions with erasures," Journal on Applied and Computational Harmonic Analysis, vol. 10, no. 3, pp. 203-233, May 2001.

[93] V. K. Goyal, M. Vetterli, and N. T. Thao, "Quantized overcomplete expansions in $\mathbb{R}^{N}$ : Analysis, synthesis, and algorithms," IEEE Transactions on Information Theory, vol. 44, no. 1, pp. 16-31, January 1998. 
[94] R. M. Gray, "Quantization noise spectra," IEEE Transactions on Information Theory, vol. 36, no. 6, pp. 1220-1244, November 1990.

[95] K. Gröchenig, "Localization of frames, Banach frames, and the invertibility of the frame operator," Journal on Fourier Analysis and Applications, vol. 10, pp. 105-132, 2004.

[96] C. S. Güntürk, "One-bit sigma-delta quantization with exponential accuracy," Communications on Pure and Applied Mathematics, vol. 56, no. 11, pp. 16081630, 2003.

[97] C. S. Güntürk, "Simultaneous and hybrid beta-encodings," in Proceedings of CISS, Princeton, NJ, March 2008.

[98] C. S. Güntürk, J. Lagarias, and V. Vaishampayan, "On the robustness of single loop sigma-delta modulation," IEEE Transactions on Information Theory, vol. 12, no. 1, pp. 63-79, January 2001.

[99] D. Han and D. R. Larson, "Frames, bases and group representations," Number 697 Memoirs AMS, Providence, RI: American Mathematical Society, 2000.

[100] B. Hassibi, B. Hochwald, A. Shokrollahi, and W. Sweldens, "Representation theory for high-rate multiple-antenna code design," IEEE Transactions on Information Theory, vol. 47, no. 6, pp. 2355-2367, 2001.

[101] R. Heath and A. Paulraj, "Linear dispersion codes for MIMO systems based on frame theory," IEEE Transactions on Signal Processing, vol. 50, no. 10, pp. 2429-2441, October 2002.

[102] C. Heil, "Linear independence of finite Gabor systems," in Harmonic Analysis and Applications, pp. 171-206, Boston, MA: Birkhäuser, 2006.

[103] C. Heil and D. Walnut, "Continuous and discrete wavelet transforms," SIAM Review, vol. 31, pp. 628-666, 1989.

[104] B. Hochwald, T. Marzetta, T. Richardson, W. Sweldens, and R. Urbanke, "Systematic design of unitary space-time constellations," IEEE Transactions on Information Theory, vol. 46, no. 6, pp. 1962-1973, 2000.

[105] R. B. Holmes and V. I. Paulsen, "Optimal frames for erasures," Linear Algebra and Its Applications, vol. 377, pp. 31-51, 2004.

[106] M. Holschneider, R. Kronland-Martinet, J. Morlet, and P. Tchamitchian, "A real-time algorithm for signal analysis with the help of the wavelet transform," in Wavelets, Time-Frequency Methods and Phase Space, pp. 289-297, Berlin, Germany: Springer-Verlag, 1989.

[107] P. Ishwar and P. Moulin, "Multiple-domain image modeling and restoration," in Proceedings of IEEE International Conference on Image Processing, pp. 362-366, Kobe, Japan, October 1999.

[108] P. Ishwar and P. Moulin, "Shift invariant restoration - An overcomplete maxent MAP framework," in Proceedings of IEEE International Conference on Image Processing, pp. 270-272, Vancouver, Canada, September 2000.

[109] P. Ishwar and P. Moulin, "On the equivalence of set-theoretic and maxent MAP estimation," IEEE Transactions on Signal Processing, vol. 51, no. 3, pp. 698-713, September 2003.

[110] P. Ishwar, K. Ratakonda, P. Moulin, and N. Ahuja, "Image denoising using multiple compaction domains," in Proceedings of IEEE International Conference on Acoustics, Speech and Signal Processing, pp. 1889-1892, Seattle, WA, 1998. 
[111] A. J. E. M. Janssen, "Duality and biorthogonality for Weyl-Heisenberg frames," Journal on Fourier Analysis and Applications, vol. 1, no. 4, pp. 403436, November 1994.

[112] R. A. Kellogg, A. Chebira, A. Goyal, P. A. Cuadra, S. F. Zappe, J. S. Minden, and J. Kovačević, "Towards an image analysis toolbox for high-throughput Drosophila embryo RNAi screens," in Proceedings of IEEE International Symposium Biomedical Imaging, pp. 288-291, Arlington, VA, April 2007.

[113] N. G. Kingsbury, "The dual-tree complex wavelet transform: A new efficient tool for image restoration and enhancement," in Proceedings of European Signal Processing Conference, pp. 319-322, 1998.

[114] N. G. Kingsbury, "Image processing with complex wavelets," Philosophical Transactions on Royal Society London A, September 1999.

[115] N. G. Kingsbury, "Complex wavelets for shift invariant analysis and filtering of signals," Journal on Applied and Computational Harmonic Analysis, vol. 10, no. 3, pp. 234-253, May 2001.

[116] N. G. Kingsbury, "Rotation-invariant local feature matching with complex wavelets," in Proceedings of European Conference on Signal Processing, Florence, Italy, September 2006.

[117] J. Kovačević and A. Chebira, "Life beyond bases: The advent of frames (Part I)," IEEE Signal Processing Magnatic, vol. 24, no. 4, pp. 86-104, July 2007.

[118] J. Kovačević and A. Chebira, "Life beyond bases: The advent of frames (Part II)," IEEE Signal Processing Magnatic, vol. 24, no. 5, pp. 115-125, September 2007.

[119] J. Kovačević, P. L. Dragotti, and V. K. Goyal, "Filter bank frame expansions with erasures," IEEE Transactions on Information Theory, vol. 48, no. 6, pp. 1439-1450, (special issue), June 2002.

[120] F. Krahmer, G. E. Pfander, and P. Rashkov, "Uncertainty in time-frequency representations on finite Abelian groups," Journal on Applied and Computational Harmonic Analysis, vol. 25, no. 2, pp. 209-225, 2008.

[121] G. Kutyniok, A. Pezeshki, A. R. Calderbank, and T. Liu, "Robust dimension reduction, fusion frames and Grassmannian packings," Journal on Applied and Computational Harmonic Analysis, to appear, 2008.

[122] D. Labate, W.-Q. Lim, G. Kutyniok, and G. Weiss, "Sparse multidimensional representation using shearlets," in Proceedings of SPIE Conference of Wavelet Applied in Signal and Image Processing, pp. 254-262, Bellingham, WA, 2005.

[123] F. Labeau, J.-C. Chiang, M. Kieffer, P. D. L., Vandendorpe, and B. Macq, "Oversampled filter banks as error correcting codes: theory and impulse noise correction," IEEE Transactions on Signal Processing, vol. 53, no. 12, pp. 46194630, December 2005.

[124] A. Laine and J. Fan, "Frame representations for texture segmentation," IEEE Transactions on Image Processing, vol. 5, no. 5, pp. 771-780, May 1996.

[125] J. Lawrence, G. E. Pfander, and D. Walnut, "Linear independence of Gabor systems in finite dimensional vector spaces," Journal on Fourier Analysis and Applications, vol. 11, no. 6, pp. 715-726, 2005.

[126] P. Lemmens and J. J. Seidel, "Equiangular lines," Journal on Algebra, vol. 24, pp. 494-512, 1973. 
[127] Y. Lu and M. N. Do, "Multidimensional directional filter banks and surfacelets," IEEE Transactions on Image Processing, vol. 16, no. 4, pp. 918-931, 2007.

[128] J. F. A. Magarey and N. G. Kingsbury, "Motion estimation using a complexvalued wavelet transform," IEEE Transactions on Signal Processing, vol. 46, no. 4, pp. 1069-1084, 1998.

[129] S. Mallat and Z. Zhang, "Characterization of signals from multiscale edges," IEEE Transactions on Pattern Analysis and Machine Intelligence, vol. 14, pp. 710-732, July 1992.

[130] H. S. Malvar, "A modulated complex lapped transform and its applications to audio processing," in Proceedings of IEEE International Conference Acoustics, Speech and Signal Processing, pp. 1421-1424, Phoenix, AZ, March 1999.

[131] J. L. Massey and T. Mittelholzer, "Welch's bound and sequence sets for code-division multiple-access systems," in Sequences II: Methods in Communication, Security and Computer Sciences, pp. 63-78, New York, NY: Springer-Verlag, 1993.

[132] J. L. Massey and M. K. Sain, "Inverses of linear sequential circuits," IEEE Transactions on Computers, vol. C-17, pp. 330-337, 1968.

[133] N. J. Munch, "Noise reduction in tight Weyl-Heisenberg frames," IEEE Transactions on Information Theory, vol. 38, no. 2, pp. 608-616, March 1992.

[134] B. A. Olshausen and D. J. Field "Sparse coding with an overcomplete basis set: A strategy employed by V1?" Vision Research, vol. 37, pp. 311-325, 1997.

[135] "Sensing, sampling, and compression," IEEE Signal Processing Magazine, vol. 25, no. 2, March 2008.

[136] J. Portilla and E. P. Simoncelli, "A parametric texture model based on joint statistics of complex wavelet coefficients," International Journal on Computes Vision, vol. 40, no. 1, pp. 49-71, December 2000.

[137] J. Portilla, M. Wainwright, V. Strela, and E. Simoncelli, "Image denoising using a scale mixture of Gaussians in the wavelet domain," IEEE Transactions on Image Processing, vol. 12, no. 11, pp. 1338-1351, November 2003.

[138] J. Princen, A. Johnson, and A. Bradley, "Subband transform coding using filter bank designs based on time domain aliasing cancellation," in Proceedings of IEEE International Conference on Acoustics, Speech and Signal Processing, pp. 2161-2164, Dallas, TX, April 1987.

[139] M. Püschel and J. Kovačević, "Real, tight frames with maximal robustness to erasures," in Proceedings of Data Compression Conference, pp. 63-72, Snowbird, UT, March 2005.

[140] G. Rath and C. Guillemot, "Frame-theoretic analysis of DFT codes with erasures," IEEE Transactions on Signal Processing, vol. 52, no. 2, pp. 447-460, February 2004.

[141] J. M. Renes, R. Blume-Kohout, A. Scot, and C. Caves, "Symmetric informationally complete quantum measurements," Journal on Mathematical Physics, vol. 45 , no. 6 , pp. 2171-2180, 2004.

[142] C. Rozell, "Distributed processing in frames for sparse approximation," in Proceedings of CISS, Princeton, NJ, March 2008. 
[143] M. Rudelson and R. Vershynin, "Geometric approach to error correcting codes and reconstruction of signals," International Mathematical Reseach Notices, vol. 64, pp. 4019-4041, 2005.

[144] I. W. Selesnick, "The double density DWT," Wavelets in Signal and Image Analysis, Kluwer Academic Publishers, 2001.

[145] I. W. Selesnick, "The design of approximate Hilbert transform pairs of wavelet bases," IEEE Transactions on Signal Processing, vol. 50, no. 5, pp. 1144-1152, May 2002.

[146] I. W. Selesnick, "The double-density dual-tree DWT," IEEE Transactions on Signal Processing, vol. 52, no. 5, pp. 1304-1314, May 2004.

[147] I. W. Selesnick, R. G. Baraniuk, and N. G. Kingsbury, "The dual-tree complex wavelet transform," IEEE Signal Processing Magazine, November 2005.

[148] P. W. Shor, "The adaptive classical capacity of a quantum channel, or information capacities of three symmetric pure states in three dimensions," 2002, http://www.citebase.org/cgi-bin/citations?id=oai:arXiv. org:quant-ph/0206058.

[149] E. P. Simoncelli, Simoncelli Web Site. http://www.cns.nyu.edu/ eero/ steerpyr/.

[150] E. P. Simoncelli, W. T. Freeman, E. H. Adelson, and D. J. Heeger, "Shiftable multiscale transforms," IEEE Transactions on Information Theory, Wavelet Transforms and Multiresolution Signal Analysis, vol. 38, no. 2, pp. 587-607, March 1992.

[151] E. Šoljanin, "Tight frames in quantum information theory," in Proceedings on DIMACS Workshop Source Coding and Harmonic Analysis, Rutgers, NJ, May 2002. http://dimacs.rutgers.edu/Workshops/Modern/ abstracts.html\#soljanin.

[152] E. Šoljanin, "Network multicast with network coding," IEEE Signal Processing Magazine, Lecture notes, 2008.

[153] E. Šoljanin, 2004, http://cm.bell-labs.com/who/emina/.

[154] E. Šoljanin, 2008, Private communication.

[155] J.-L. Starck, M. Elad, and D. L. Donoho, "Redundant multiscale transforms and their application for morphological component separation," Advances in Imaging and Electron Physics, vol. 132, 2004.

[156] T. Strohmer, "Finite and infinite-dimensional models for oversampled filter banks," in Modern Sampling Theory: Mathematics and Applications, pp. 297320, Boston, MA: Birkhäuser, 2000.

[157] T. Strohmer and R. Heath, "Grassmannian frames with applications to coding and communications," Journal on Applied and Computational Harmonic Analysis, vol. 14, no. 3, pp. 257-175, 2003.

[158] M. Sustik, J. A. Tropp, I. S. Dhillon, and R. W. Heath, Jr., "On the existence of equiangular tight frames," Linear Algebra and Its Applications, vol. 426, no. 2-3, pp. 619-635, 2007.

[159] I. E. Telatar, "Capacity of multiantenna Gaussian channels," European Transactions on Telecommunications, vol. 10, no. 6, pp. 585-595, November 1999.

[160] J. A. Tropp, "Greed is good: Algorithmic results for sparse approximation," IEEE Transactions on Information Theory, vol. 50, no. 10, pp. 2231-2242, October 2004. 
[161] J. A. Tropp, I. S. Dhillon, R. Heath, Jr., and T. Strohmer, "Designing structured tight frames via an alternating projection method," IEEE Transactions on Information Theory, vol. 51, no. 1, pp. 188-209, January 2005.

[162] M. Unser, "Texture classification and segmentation using wavelet frames," IEEE Transactions on Image Processing, vol. 4, pp. 1549-1560, November 1995.

[163] P. P. Vaidyanathan, Multirate Systems and Filter Banks. Englewood Cliffs, NJ: Prentice Hall, 1992.

[164] R. Vale and S. Waldron, "Tight frames and their symmetries," Constructive Approximation, vol. 21, pp. 83-112, 2005.

[165] R. Vershynin, "Frame expansions with erasures: An approach through the non-commutative operator theory," Journal on Applied and Computational Harmonic Analysis, vol. 18, pp. 167-176, 2005.

[166] M. Vetterli and J. Kovačević, Wavelets and Subband Coding. Signal Processing, Englewood Cliffs, NJ: Prentice Hall, 1995. http://waveletsandsubbandcoding.org/.

[167] M. Vetterli, J. Kovačević, and V. K. Goyal, The World of Fourier and Wavelets: Theory, Algorithms and Applications. http://www. fourierandwavelets.org/, 2008.

[168] P. Viswanath and V. Anantharam, "Optimal sequences and sum capacity of synchronous CDMA systems," IEEE Transactions on Information Theory, vol. 45, no. 6, pp. 1984-1991, September 1999.

[169] S. Waldron, "Generalised Welch bound equality sequences are tight frames," IEEE Transactions on Information Theory, vol. 49, no. 9, pp. 2307-2309, September 2003.

[170] J. B. Weaver, X. Yansun, D. M. Healy, Jr., and L. D. Cromwell, "Filtering noise from images with wavelet transforms," Magnetic Resonance in Medicine, vol. 21, no. 2, pp. 288-295, 1991.

[171] L. Welch, "Lower bounds on the maximum cross correlation of signals," IEEE Transactions on Information Theory, vol. 20, pp. 397-399, 1974.

[172] A. Witkin, "Scale-space filtering," in Proceedings of International Joint Conference on Artificial Intelligence, pp. 1019-1022, 1983.

[173] J. K. Wolf, "Redundancy, the discrete Fourier transform and impulse noise cancellation," IEEE Transactions on Communications, vol. 31, no. 3, pp. 458461, March 1983.

[174] J. M. Wozencraft and I. M. Jacobs, Principles of Communication Engineering. Waveland Press, Inc., 1965.

[175] P. Xia, S. Zhou, and G. Giannakis, "Achieving the Welch bound with difference sets," IEEE Transactions on Information Theory, vol. 51, no. 5, pp. 19001907, May 2005.

[176] X.-G. Xia, "New precoding for intersymbol interference cancellation using nonmaximally decimated multirate filterbanks with ideal FIR equalizers," IEEE Transactions on Signal Processing, vol. 45, no. 10, pp. 2431-2441, October 1997. 


\section{References}

[177] Y. Xu, J. B. Weaver, D. M. Healy, Jr., and J. Lu, "Wavelet transform domain filters: A spatially selective noise filtration technique," IEEE Transactions on Image Processing, vol. 3, no. 6, pp. 747-758, December 1994.

[178] B.-J. Yoon and H. S. Malvar, "Coding overcomplete representations of audio using the MCLT," in Proceedings of Data Compression Conference, pp. 152161, Snowbird, UT, March 2008. 